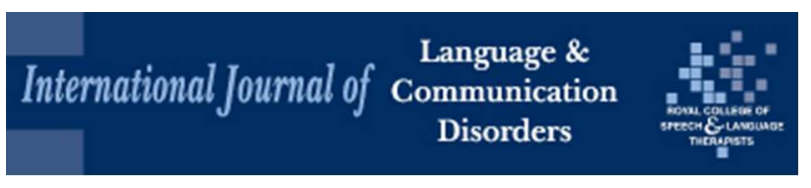

\title{
Service delivery and intervention intensity for phonology- based speech sound disorders
}

\begin{tabular}{|r|l|}
\hline Journal: & International Journal of Language \& Communication Disorders \\
\hline Manuscript ID & TLCD-2017-0180.R1 \\
\hline Wiley - Manuscript type: & Review \\
\hline Keywords: & speech, evidence based practice (EBP), phonology, children, intervention \\
\hline \multicolumn{2}{|l}{} \\
\hline
\end{tabular}

SCHOLARONE ${ }^{\text {Ix }}$

Manuscripts 


\begin{abstract}
Background: When planning evidence-based intervention services for children with phonologybased speech sound disorders (SSD), speech and language therapists (SLTs) need to integrate research evidence regarding service delivery and intervention intensity within their clinical practice. However, relatively little is known about the optimal intensity of phonological interventions, and whether SLTs' services align with the research evidence.

Aims: The aims of this paper are twofold. First, to review external evidence (that is, empirical research evidence external to day-to-day clinical practice) regarding service delivery and intervention intensity for phonological interventions. Second, to investigate SLTs' clinical practice with children with phonology-based SSD in Australia, focussing on service delivery and intensity. By considering these two complementary sources of evidence, SLTs and researchers will be better placed to understand the state of the external evidence regarding the delivery of phonological interventions and appreciate the challenges facing SLTs in providing evidencebased services.
\end{abstract}

Methods: Two studies are presented. The first is a review of phonological intervention research published between 1979 and 2016. Details regarding service delivery and intervention intensity were extracted from the 199 papers that met inclusion criteria identified through a systematic search. The second study was an online survey of 288 SLTs working in Australia, focused on the service delivery and intensity of intervention provided in clinical practice.

Main contributions: There is a gap between the external evidence regarding service delivery and intervention intensity and the internal evidence from clinical practice. Most published intervention research has reported to provide intervention 2-3 times per week in individual sessions delivered by an SLT in a university clinic, in sessions lasting 30-60 minutes comprising 
100 production trials. SLTs reported providing services at intensities below that found in the literature. Further, they reported workplace, client, and clinician factors that influenced the intensity of intervention they were able to provide to children with phonology-based SSD.

Conclusions: Insufficient detail in the reporting of intervention intensity within published research coupled with service delivery constraints may affect the implementation of empirical evidence into everyday clinical practice. Research investigating innovative solutions to service delivery challenges is needed to provide SLTs with evidence that is relevant and feasible for clinical practice.

\section{What this paper adds}

What is already known on this subject? A previous review by Baker \& McLeod (2011) provided a valuable synthesis of phonological intervention research published between 1979 and 2009. However, this review did not consider the fundamental issue of dose, nor the barriers facing SLTs in delivering evidence-based intervention services. Anecdotal evidence suggests that SLTs are providing insufficient services to children with phonology-based SSD, but minimal empirical research investigating this is available.

What this paper adds? This paper provides a detailed and updated synthesis of the extant literature for phonological intervention, with a particular focus on service delivery and intervention intensity. By comparing empirical evidence with the evidence gained through a survey of clinical practice, insights about the challenges of implementing research into clinical practice are provided.

Clinical implications of this study. SLTs are encouraged to document the service delivery and intensity of the intervention they provide to children with phonology-based SSD and to work alongside researchers to generate practice-based evidence supporting their services. 


\section{Introduction}

Worldwide, speech and language therapists (SLTs) are encouraged to make clinical decisions according to the principles of evidence-based practice (Royal College of Speech \& Language Therapists, 2016, Speech Pathology Australia, 2015). This framework for clinical decision-making involves using clinical expertise to integrate the best available evidence internal to clinical practice and the preferences of a fully-informed client with the "best available external evidence from systematic research" (Dollaghan, 2007: 2). It is important that SLTs are able to make evidence-based decisions regarding the management of their caseload, as outcomes are assumed to be related to the integration and implementation of these sources of evidence (Odom, 2009). A high proportion of SLTs' caseloads comprise children with speech sound disorders (SSD; Broomfield and Dodd, 2004a).

Children with SSD may experience "any combination of difficulties with perception, articulation/motor production, and/or phonological representation of speech segments (consonants and vowels), phonotactics (syllable and word shapes), and prosody (lexical and grammatical tones, rhythm, stress, and intonation) that may impact speech intelligibility and acceptability" (International Expert Panel on Multilingual Children's Speech, 2012: 1). The most common subtype of SSD is a phonology-based SSD which involves a difficulty in learning the phonological system of the ambient language (Broomfield and Dodd, 2004b). Without the right type and amount of help during the years before a child starts school, children with SSD face an increased risk of academic and socioemotional difficulties (McCormack et al., 2011, Lewis et al., 2016). When planning intervention for these children, SLTs need to consider and integrate external (that is, empirical) evidence regarding service delivery, intervention approaches and intervention intensity within their clinical practice. However, SLTs working in clinical practice 
report having little time and resources to access, appraise and integrate research from the growing external evidence base (O'Connor and Pettigrew, 2009). One strategy to facilitate this process is to consider reviews and summaries of the literature. The external evidence base for phonological intervention has been reviewed by Baker and McLeod (2011).

In a comprehensive narrative review, Baker and McLeod (2011) examined phonological intervention research published between 1979 and 2009, and found that 46 intervention approaches had been examined in the evidence base. Of these 46 different approaches, 23 were described in more than one publication. Although phonological interventions are known to be effective (Law et al., 2004), no one approach was recommended as the most effective for all children with a phonology-based SSD. For SLTs, a decision about which approach to choose is further complicated by the varying models of service delivery used in everyday clinical practice (Pring et al., 2012). Indeed, models of service delivery and resource constraints are known to drive clinical decision-making (McCurtin and Clifford, 2015).

Issues within service delivery include how intervention is provided (for example, group or individual, or telehealth), who provides the intervention (for example, the SLT or parent), where intervention is conducted (for example, at school or at a clinic), and how much intervention is provided (intervention intensity). In their review of intervention literature for SSD Baker and McLeod (2011) showed that most external research evidence published between 1979 and 2009 was based on the following service delivery model: individual intervention, delivered by an SLT, in a university clinic. Other reviews have explored the external evidence base for different service delivery models, including parent-delivered intervention (e.g., Sugden et al., 2016). One important aspect of service delivery that has received increasing attention is intervention intensity. 
Intervention intensity encompasses dose frequency, total intervention duration, dose form, dose, and cumulative intervention intensity (Warren et al., 2007). Dose frequency refers to the number of intervention sessions provided over a period of time (for example, $1 \times$ week or $2 \times$ month), with total intervention duration being the total period over which an intervention is provided (for example, 12 weeks or 1 year). Dose form refers to the activity or task in which a teaching episode — containing the active ingredients of an intervention—are delivered (Baker, 2012), with dose being the number of times an active ingredient or teaching episode is delivered per session (for example, 100 productions trials per session). Cumulative intervention intensity is the product of dose $\times$ dose frequency $\times$ total intervention duration, a construct which provides a "useful general indicator of overall intensity" (Warren et al., 2007: 72). The narrative review by Baker and McLeod (2011) showed that most published evidence for phonological intervention reports a dose frequency of 2-3 times per week, in sessions lasting 30-60 minutes. Other reviews have also emphasised the importance of dose frequency for positive outcomes following phonological intervention (Kaipa and Peterson, 2016). Regarding the total duration of intervention needed for discharge from speech therapy services, the evidence is less expansive. Where it has been reported, total intervention duration has ranged from 7 to 46 months, with a mean duration of approximately 12 months (Baker and McLeod, 2011). Although providing a valuable synthesis of some elements of intervention intensity for phonological intervention approaches, the narrative review by Baker and McLeod (2011) did not consider the dose of intervention provided within these research studies. This is currently unknown yet essential to the conduct of evidence-based practice.

The increased interest regarding optimal intervention intensity for phonology-based SSD (Williams, 2012, Baker, 2012) in combination with the essential role that dose plays in treatment 
outcomes, warrants a review and synthesis of this evidence. As stated by To et al. (2012: 465), the limited available evidence regarding intervention intensity "leads to difficulties in establishing guidelines on treatment intensity for SLTs when managing SSDs”. If SLTs are to make evidence-based clinical decisions to optimise children's intervention outcomes, recommendations from the empirical evidence, particularly on intervention intensity, are needed. Moreover, if the goal is for implementation of this evidence into clinical practice, the evidence needs to be examined in light of the services that SLTs currently provide. Therefore, the purpose of this paper is twofold. First, to examine the evidence base for intensity in phonological interventions, with a particular focus on dose. Second, to report the results of a survey of SLTs' clinical practice and the intensity of services they provide to children with phonology-based SSD. The results of these two studies are then contrasted, to facilitate an understanding of how the external evidence is applied within clinical practice. By considering these two sources of evidence - external research evidence and internal evidence from day-to-day clinical practiceSLTs and researchers will be better positioned to understand the state of the empirical evidence regarding the provision of phonological intervention and appreciate the challenges facing SLTs as they strive to provide evidence-based services.

\section{Study 1: A Review of the Evidence}

\section{Method}

A systematic search and review was conducted. According to Grant and Booth (2009), this type of review is appropriate for synthesising a large body of research evidence, and contrasts with systematic reviews by including a variety of study designs.

Search strategy. The following online databases were searched: Medline, Cumulative Index to Nursing and Allied Health Literature (CINAHL), Education Resources Information 
Centre (ERIC), Scopus, Linguistic and Language Behaviour Abstracts (LLBA), SpeechBITE and the American-Speech-Language-Hearing Association's (ASHA's) online journal search site. The following search terms were used: phonological OR phonology OR articulation OR speech sound disorder AND intervention OR therapy OR treatment. The reference lists of included papers were hand-searched for additional papers. Additionally, all papers contained in reviews by Baker and McLeod (2011) and Sugden et al. (2016) were included in this study.

Inclusion criteria. Papers met the following inclusion criteria:

- Peer-reviewed paper written or translated into English published between 1979 and 2016;

- Reported on phonological intervention/therapy/treatment research for children identified as having a phonological/articulation/speech impairment/delay/disorder with or without concomitant difficulties such as developmental language disorder, hearing loss, cleft-lip and/or palate, and/or stuttering;

- Research design corresponding to the ASHA (2004) level-of-evidence categories 1 (meta-analysis) to level III (case studies).

Exclusion criteria. Papers were excluded if they:

- Were level IV (expert opinion pieces) according to ASHA (2004) level-of evidence categories

- Reported on studies that had previously been published (and thus were already included in the review) and did not present new data, hypotheses or conclusions.

Data extraction and analysis. Data extraction was conducted by the first two authors. The following data was extracted from all papers that met the inclusion criteria and entered into a Microsoft Excel ${ }^{\circledR}$ spreadsheet: authors, year of publication, journal name, country where research was conducted (if not explicitly stated within the manuscript, the location of the first 
author's institutional affiliation was entered), participant numbers and age, intervention approach, and study design. Several papers reported on two or more studies: where this was the case, information about participant numbers, intervention approaches and study design were coded separately for each study. In keeping with the review by Baker and McLeod (2011), for studies that did not provide an explicit name for intervention delivered to children identified as having a phonological delay/disorder/impairment, the term generic phonological approach was used.

Following this process, information about service delivery and intervention intensity was extracted for all studies that were not classified as reviews. Review papers were not subject to further data extraction and analysis as they collate studies rather than report on specific investigations. The information extracted about service delivery included: how intervention was delivered (e.g. individually or in groups), the primary intervention agent, and where intervention was delivered. Information about intervention intensity was extracted according to the categories identified by Warren et al. (2007), including dose, dose frequency, total intervention duration (in weeks and number of sessions), and cumulative intervention intensity. Information on dose form was not extracted, as this was deemed to have been extracted under intervention approach coded previously. In addition to the categories of intervention intensity presented by Warren et al. (2007), we extracted information on session duration, and whether intervention was delivered over a restricted period or until the child(ren) were seen from the point of initial referral to a specific service until discharge.

Throughout the data extraction process, it became apparent that one category for dose was insufficient to encompass the range of information that was reported in the literature. Thus, this category was expanded to include three different types of dose: production dose, akin to the 
number of attempts a child had to produce their targets within a session; perception dose, akin to the number of times a child completes focussed perceptual or input-based tasks (such as auditory bombardment or auditory discrimination tasks), and; conceptual dose, which included the number of times a child completed conceptual-type activities, such as phonological awareness or metaphonological tasks.

Several decisions relating to data extraction were made. First, only information reported in each paper was extracted, even if further details were available elsewhere. Second, when information regarding service delivery or intervention intensity presented in a paper was unclear or ambiguous, these categories were coded conservatively as not reported or unclear. Third, the focus of the review was on SLT-delivered interventions: as such, only SLT-delivered services were coded for intensity. Finally, for studies explicitly investigating comparisons in service delivery and/or intensity, both models of service delivery and/or intensity were coded so as to capture the range of these models within the literature.

Reporting of intervention intensity. In light of the importance of reporting intensity for implementation and replication of interventions (Hoffmann et al., 2014), an appraisal of the reporting of intervention intensity was conducted for all studies that were not identified as a review paper. Studies received a score out of 7, with one point allocated for sufficient reporting of each of the following components of intervention intensity to enable replication: dose, dose form, dose frequency, session duration, total intervention duration (in weeks or months), total intervention duration (in sessions), and cumulative intervention intensity.

Reliability. The second author re-coded 20 (10.1\%) randomly selected papers. Interjudge reliability was $97.5 \%$. The first author re-coded the same papers: intra-judge reliability was $96.8 \%$. 


\section{Results}

Of the 6584 papers identified in the search process, 199 papers matched the inclusion criteria. Details of the included papers are provided in Appendix A. Figure 1 shows the year of publication of the 199 papers, which came from the following countries: the US ( $n=109$, $54.8 \%)$, the UK $(n=27,13.6 \%)$, Canada $(n=18,9.0 \%)$, Australia $(n=16,8.0 \%)$, Brazil $(n=$ 15, 7.5\%), New Zealand ( $n=4,2.0 \%)$, Mexico $(n=3,1.5 \%)$, Portugal $(n=2,1.0 \%)$, Iran $(n=1$, $0.5 \%)$, Norway $(n=1,0.5 \%)$, Sweden $(n=1,0.5 \%)$ and Turkey $(n=1,0.5 \%)$. One paper $(0.5 \%)$ reported on a study conducted in both the US and New Zealand. The 199 papers included 211 studies. These studies had the following designs: review $(n=5,2.4 \%)$, randomised controlled trial (RCT; $n=34,16.1 \%)$, non-randomised controlled trial $(n=14,6.6 \%)$, quasi-experimental group design ( $n=27,12.8 \%)$, single-case experimental design (SCED; $n=70,32.7 \%$ ), and case studies $(n=61,29.4 \%)$. Fifty-eight intervention approaches were identified in these studies, the most common of which were: minimal pairs (in 51 studies), a collection of approaches based on the principles of complexity (in 38 studies, such as maximal oppositions, treatment of the empty set, and intervention targeting complex onsets), a generic phonological approach (in 34 studies), traditional articulation therapy (in 14 studies), and a modified cycles approach (in 12 studies). Of these 58 approaches, 26 had been investigated in more than one study.

\section{[INSERT FIGURE 1 ABOUT HERE]}

One-hundred and ninety-four papers comprising 206 studies reported on specific investigations or cases of phonological intervention. These studies included between 1 and 730 participants (average $=16.7$, median $=6$, mode $=1)$ who were between 18 and 144 months in age (average minimum age $=49.8$ months, average maximum age $=67.4$ months). Sixty-five 
$(31.6 \%)$ of these studies included at least one child with a concomitant disorder such as hearing impairment, cleft lip and/or palate, language disorder, or stuttering.

Service delivery. The service delivery models used in the 206 studies reporting on phonological intervention are presented in Table 1 . The most common service delivery model used within the literature was individual intervention (75.5\% of studies) delivered by an SLT $(86.8 \%)$ in a university clinic $(54.7 \%)$.

[INSERT TABLE 1 ABOUT HERE]

Intervention intensity. The frequency and duration of intervention sessions are presented in Table 2. Dose frequency ranged from once every 6 weeks (e.g., the parent group from experiment 2 in Lancaster et al., 2010) to daily (e.g., Pamplona et al., 2014), with the majority of studies $(55.2 \%)$ reporting a dose frequency of 2 to $3 \times$ weekly. Two studies reported a dose frequency of "biweekly", which was coded as unclear due to the potential for misinterpretation of $2 \times$ week or once every 2 weeks. Sessions ranged in duration from 15 minutes (e.g., Dunn and Barron, 1982) to a 2-day workshop (e.g., Study 2 from Dodd and Barker, 1990), with $70.3 \%$ of studies $(n=149)$ reporting a session duration of between 30 and 60 minutes.

[INSERT TABLE 2 ABOUT HERE]

Fifty-one studies (24.8\%) reported some information about dose, with 155 studies (75.2\%) not reporting any information regarding dose. Of these 51 studies, 42 (20.4\% of all studies) provided dosage information about all of the interventions included within the study, with the remaining 9 studies providing information only about some of the intervention that was delivered. The minimum, maximum and average production, perception and conceptual dose provided within a session are provided in Table 3 . The most commonly reported production dose 
was 100 trials per session (29.8\% of studies reporting on production dose), which was typically delivered in sessions lasting 30 to 45 minutes. Regarding perception dose, 30 trials was the most commonly reported (in $20.0 \%$ of the studies reporting perception dose). Although many studies reported including conceptual tasks in intervention (e.g., Gillon, 2000), no studies provided information about the conceptual dose provided within a session.

\section{[INSERT TABLE 3 ABOUT HERE].}

Twelve studies $(5.7 \%)$ reported on the total duration of intervention provided to children with phonology-based SSD from initial assessment to discharge. These studies reported a total intervention duration of between 12 and 184 weeks (average $=61.3$ weeks), comprising between 10 and 105 sessions (average $=43.8$ sessions) provided until discharge. The majority of studies ( $n=197,92.9 \%)$ provided intervention over a restricted duration (for example, a pre-determined number of weeks or sessions), with three studies (1.4\%) not reporting whether intervention was delivered over a restricted duration or not.

It was not possible to calculate the cumulative intensity of intervention provided in studies reporting on the total duration of intervention from initial assessment to discharge. For instance, of the twelve studies that reported providing intervention until discharge from services, none provided sufficient information about dose to enable calculation of the cumulative intervention intensity received by the participants. Thus, the cumulative intensity required for discharge from phonological intervention remains unknown. That noted, one study did indicate that four out of nine children were discharged after receiving school-based group intervention services for between 66 and 100 hours (Montgomery and Bonderman, 1989). For studies reporting on intervention provided over a restricted duration ( $92.9 \%$ of all studies), limited 
reporting coupled with heterogeneity in methods, intervention, and outcomes prohibited calculation of cumulative intervention intensity.

Reporting of intervention intensity. All 206 studies that were not coded as reviews reported dose form, with 176 studies (85.4\%) reporting dose frequency, 170 studies $(82.5 \%)$ reporting session duration, 158 studies $(76.7 \%)$ reporting total intervention duration of the study in weeks or months, $148(71.8 \%)$ studies reporting total intervention duration in sessions, 42 studies (20.4\%) reporting dose, and 7 studies (3.4\%) reporting cumulative intervention intensity.

Most studies reported either $5(n=84,40.8 \%)$ or $4(n=66,32.0 \%)$ elements of intervention intensity. Two studies (1.0\%) reported only dose form (Penney et al., 1994, Mota et al., 2007). Two studies reported sufficient information about all 7 elements of intensity to enable replication (Allen, 2013; Gildersleeve-Neumann and Goldstein, 2015). There was a trend for studies using a SCED design to report more detail regarding intervention intensity than reported in RCTs; $23.2 \%$ of SCED studies reported 6 or 7 elements of intensity, compared to $11.8 \%$ of RCTs.

\section{Discussion of Study 1}

This review presented an updated synthesis of the evidence base for phonological intervention and offered new insights into intervention intensity. Specifically, this was the first study to synthesise the external evidence regarding the fundamental concept of dose in phonological intervention research. Of the 199 papers that were identified, 194 papers comprising 206 studies reported on children's speech outcomes following intervention. Multiple study designs, primarily single-case research and case studies, and 58 intervention approaches were used across the evidence base. Echoing findings from the review by Baker and McLeod (2011), we found that the most common intervention approaches used within the evidence base 
were minimal pairs and intervention based on principles of complexity, while the most common service delivery model used was individual intervention, delivered by an SLT in a university clinic. Although the present review included an additional 65 papers than the review by Baker and McLeod (2011), the evidence for phonological intervention still primarily reports a dose frequency of 2 to $3 \times$ week in sessions lasting 30 to 60 minutes. No additional evidence exploring the total duration of intervention required to remediate an SSD from initial assessment to discharge has been published: thus, the evidence for the total amount of intervention needed for children to be discharged with intelligible speech remains unclear.

The present review extended on the findings made by Baker and McLeod by investigating intervention intensity in more detail. When it was reported, the most common dose was 100 production trials within a 30 to 45 minute session. The findings of this review highlight the limited and often insufficient reporting of intervention intensity within the research base for phonological interventions. As stated by Sommers et al. (1992: 19), "the frequency of reporting only limited information [about intervention intensity] or not reporting at all was alarming". It is even more alarming that the reporting of intervention intensity has not substantially improved in the 25 years since.

Although limited reporting of intervention intensity restricts the application of research into clinical practice (Hoffmann et al., 2014), the design of some intervention research may preclude sufficient reporting. The current study identified that SCED research tends to report more information about intensity than larger group designs, such as RCTs. RCTs in the field of speech and language therapy are known to be problematic for the reporting of interventions (Ludemann et al., 2017); while the reasons for this are unclear, it may be that authors of RCTs focus on reporting the scientific methods of a study whereas authors of SCED research may 
instead focus on an individual's response to intervention, requiring more detailed reporting of the intervention delivered. Although understandable in certain contexts, limited details in the reporting of intervention research impacts the useability of research evidence for clinicians and researchers.

It is important to note that the service delivery models and schedules of intervention intensity reported within the evidence base may not be optimal for treating children with phonology-based SSD: rather, the use of these models may have occurred for a range of reasons, including resource constraints, customary practice patterns, or personal preferences of those conducting the research. The heterogeneity within the evidence base regarding research design, intervention approach, and participant details further complicates clear interpretation of optimal intervention intensity for children with phonology-based SSD.

When the findings of this review are combined with other sources of evidence, however, SLTs have some guidelines on which to base their service delivery and intervention intensity. For example, evidence from US-based SLTs' clinical practice suggests that individual intervention provides better outcomes for children with SSD than group intervention (American Speech-Language-Hearing Association, 2011). Although the optimal intensity of intervention for SSD is unknown (Baker, 2012), randomised-controlled trial evidence suggests a higher dose frequency of $3 \times$ per week yields better speech outcomes for children with phonology-based SSD than intervention delivered $1 \times$ per week (Allen, 2013). Further, in a retrospective analysis of outcomes from phonological interventions, Williams (2012) reported that a minimum dose of 50 trials in a 30 minute session was needed for intervention to be effective for children with moderate-severe phonology-based SSD. For children with severe disorders, this increased to a minimum of 70 trials in a 30 minute session. Limited evidence exploring the total duration of 
intervention or cumulative intervention intensity is available. Considering the available evidence, however, it appears that—when treating phonology-based SSD—SLTs should strive to deliver individual intervention, 2-3 times per week, in sessions lasting 30-60 minutes comprising at least 50 to 100 production trials. Unfortunately, this model of practice may not be possible for many SLTs worldwide, who need to consider the realities of clinical practice including limited funding, resources, and time.

\section{Study 2: Survey of Australian SLTs' Clinical Practice}

In an ideal world, clinical decisions about service delivery and intervention intensity would be grounded in evidence-based practice. Yet clinical decision-making is not straightforward, and SLTs face barriers - including those related to the workplace, client and the clinician - to implementing external evidence within their practice (Lim et al., 2017, Brandel and Loeb, 2011). Such barriers may include workload or caseload size, availability of intervention resources, client disorder type and severity, and an SLT's professional training and continuing development opportunities. Combined, these barriers paint a complex picture around what influences evidence-based decision-making with regards to the service delivery and intervention intensity provided to children with phonology-based SSD. It would be useful, then, to consider how the external evidence for phonological interventions is applied within a specific context. One country with a long history of speech and language therapy services and a penchant for evidence-based practice is Australia.

\section{What is Known About Intervention Services for Children With SSD in Australia?}

Intervention services in Australia are usually provided through the health, education or private sector; however, there is no unifying piece of legislation mandating access to services for children, and eligibility criteria differ between states and territories (McLeod et al., 2010). Given 
the large and increasing demand for these services, they are often limited (for example, by providing a limited number of sessions before discharge, or provided only to children who have not yet started school) and families may face a long wait (commonly 12 months) to access these services (Speech Pathology Australia, 2014, Ruggero et al., 2012). Due to the limited services available to children with SSD, many families seek services from the fast-growing private sector; however, the costs of these services can be prohibitive (Senate Community Affairs References Committee, 2014, Speech Pathology Australia, 2014). Access to services can be difficult for families living in rural or remote areas of Australia, who often have to travel long distances for infrequent or insufficient services (Verdon et al., 2011). Thus, access to services for SSD is dependent on where — and in which state or territory—children live, in addition to their family's economic situation. What do these services typically look like?

McLeod and Baker (2014) surveyed 231 Australian SLTs about their service delivery when working with children with SSD. They found that SLTs most commonly report providing individual intervention (95.7\% of respondents) that is delivered by the SLT (91.4\%) in a clinic setting (73.8\%). Other common service delivery models included parent training (68.6\%) and provision of a home program (64.8\%). Just over half (57.6\%) of the SLTs reported providing services within preschools or schools. Two-thirds of the SLTs reported having a waiting list for services. The SLTs who completed this survey reported using a wide range of intervention approaches, commonly auditory discrimination, minimal pairs, Cued Articulation, phonological awareness, traditional articulation therapy, auditory bombardment, the Nuffield Centre Dyspraxia Programme and core vocabulary (McLeod and Baker, 2014). When compared with the findings from Study 1, above, this survey provides evidence that the typical service delivery models and intervention approaches used to treat SSD in Australia broadly reflect the research 
base. However, this survey did not consider the intensity of intervention provided to these children. This is currently unknown. Further, the study by McLeod and Baker (2014) did not investigate the factors - workplace, client or clinician — that may shape the delivery of services. These, too, are unknown. Thus, there is a need to examine both the intensity of services delivered to children with SSD and the barriers faced by SLTs that may influence services. By comparing and integrating this information with the results of Study 1, above, insights about the implementation of external evidence within clinical practice may be gained.

\section{Method}

Development of the survey instrument. This questionnaire contained 67 questions covering the following topics: general caseload questions, demographic information, service delivery, intervention approaches, intervention intensity, target selection, practices when working with families and the provision of home practice. The results of the final two of these topics (working with families and home practice) are presented elsewhere (details removed for peer review). See Appendix B for the questions reported in this paper. The questions were adapted from previous surveys of clinical practice (e.g. Joffe and Pring, 2008, Skahan et al., 2007, McLeod and Baker, 2004, Watts Pappas et al., 2008). This questionnaire contained multiple choice, yes/no and open response fields.

Procedures. A pilot version of the questionnaire was sent to five Australian paediatric SLTs who were asked to comment on the overall design of the survey. This process resulted in minimal changes to the survey instrument. The final version of the questionnaire was then administered through the online survey host SurveyMonkey ${ }^{\circledR}$. The survey was anonymous, and was open for two periods each of 8-weeks duration commencing in October 2014 and again in March 2015. The first author contacted organisations (such as Speech Pathology Australia, 
national special interest groups related to SSD, and Australian universities that offer courses in speech and language therapy) who were asked to distribute information about the survey to their networks. Additionally, information about the survey was posted on social media sites (including Twitter and speech and language therapy-related Facebook groups). More detailed information about recruitment and the administration of this survey is provided in (details removed for peer review). Ethical approval was obtained (details removed for peer review).

Participants. The target population for this survey were SLTs working in Australia with children with phonology-based SSD. This survey was attempted by 335 Australian SLTs; however, $14.1 \%$ of these $(n=47)$ completed only the first page of the survey which asked about demographic information. Given that these responses did not provide information useful to the aims of the survey, they were not included in the analyses. Thus, a total of 288 responses were analysed. Their demographic information is presented in Table 4.

\section{[INSERT TABLE 4 ABOUT HERE]}

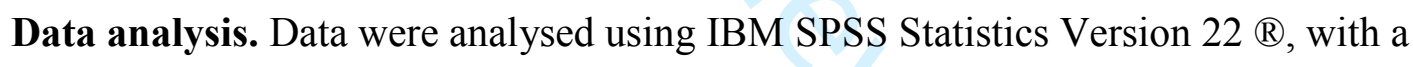
codebook used to record decisions related to data analysis. Descriptive statistics are reported. Some participants did not answer all of the questions within the questionnaire; where percentages are presented within this paper, they were calculated after excluding these missing responses (i.e., percentages are for valid data only). The total number of valid responses is provided within the text or a table.

\section{Results}

Caseload. The SLTs reported to have between zero to 800 children on their caseload (valid responses $n=275$ ), with a median of 41 (interquartile range $=25-70$ ). Participants reported that between zero and 188 children on their caseload had a primary diagnosis of 
phonology-based SSD (median $=11$, interquartile range $=5-25)$. SLTs were asked to provide the most common age of children with SSD on their caseload: 55 months was the average response. Other information regarding the caseloads of SLTs who completed this questionnaire is available elsewhere (details removed for peer review).

Service delivery. The service delivery models reported to be used by SLTs who participated in this survey are presented in Table 5. Individual intervention (96.4\%) delivered by an SLT $(97.8 \%)$ was the most common service delivery model, which was delivered in a range of locations.

\section{[INSERT TABLE 5 ABOUT HERE]}

Intervention approaches. Participants were asked to identify the intervention approaches they usually use with children with phonology-based SSD; results are shown in Table 6. Minimal oppositions contrast (minimal pairs) and auditory discrimination approaches were the most commonly used approaches (by $83.0 \%$ and $75.6 \%$ of SLTs, respectively). SLTs were also asked to select the type of treatment targets they usually select when treating phonology-based SSD. A majority (52.4\%) reported usually selecting developmental targets, with $20.4 \%$ reporting to select collapse of contrast targets, $20.8 \%$ reporting to select nondevelopmental targets, and 6.3\% reporting to select other types of targets (valid responses $n=$ 269). Participants were also asked to identify if they follow a hierarchy of sound production (starting in isolation and progressing through syllables and words to conversation) when treating phonology-based SSD or if they focus on error patterns within a child's speech: $50.2 \%$ of SLTs reported that they focussed on error patterns, with $42.0 \%$ reporting to follow a hierarchy, and $7.8 \%$ reporting "other" (valid responses $n=269$ ). Additionally, participants were asked to indicate the types of tasks they usually include in intervention for phonology-based SSD: $98.9 \%$ 
indicated they usually include production tasks, $86.2 \%$ reported that they usually include perception tasks, $66.9 \%$ reported that they usually included conceptual tasks, and $4.5 \%$ indicated that they usually include other tasks (valid responses $n=269$ ). The definitions of these tasks provided to participants are included in Appendix B

\section{[INSERT TABLE 6 ABOUT HERE]}

Intervention intensity. Results regarding dose frequency and session duration reported to be provided are presented in Table 7. Most SLTs reported providing intervention $1 \times$ week (62.3\%) for 30 to 44 minutes (62.4\%). Regarding dose, SLTs were asked to select the number of production trials, perception trials and conceptual trials provided to children within each intervention session. Results are presented in Table 8. SLTs reported providing between 21-49 (37.6\%) and 50-99 (39.8\%) production trials within a session. The SLTs reported providing more production trials within each session than either perception or conceptual trials.

\section{[INSERT TABLE 7 ABOUT HERE]}

\section{[INSERT TABLE 8 ABOUT HERE]}

Participants reported that children received a total intervention duration of 4 to 156 weeks, with an average of $38.2(S D=28.0$; valid response $n=203)$. Participants were also asked to report the total number of sessions provided to children with phonology-based SSD. SLTs reported that children received between 2 and 400 sessions, with an average of 22.7 ( $S D=30.68$; valid responses $n=281)$. Most SLTs who completed this survey (65.2\%) indicated that the majority of children on their caseload receive the same intensity of intervention (valid responses $n=256$ ). Almost a quarter of SLTs reported that they provide blocks of intervention to children with phonology-based SSD (22.3\%; valid responses $n=265)$, which most commonly involved children receiving 10 weeks of intervention (23.4\%) with 10 weeks off (22.1\%) between blocks. 
Participants were asked to identify factors that influence the intensity of intervention that they provide. Results are presented in Table 9. Participants were asked to identify which of workplace, client and clinician factors had the biggest influence on the intensity of intervention they provide to children with phonology-based SSD. The majority of participants reported that workplace factors had the biggest influence (52.8\%), followed by client (43.9\%) and clinician factors $(3.3 \%$; valid responses $n=246)$.

Finally, participants were asked to identify their ideal intensity of intervention (including dose frequency, session duration, and total intervention duration in weeks and number of sessions) for a preschool-aged child with a moderate-severe phonology-based SSD. Results for dose frequency and session duration are shown in Table 10. Most SLTs (50.9\%) reported an ideal dose frequency of 2-3 $\times$ week, in sessions lasting 30 to 60 minutes $(88.7 \%)$. Regarding ideal total intervention duration, SLTs reported that they would ideally deliver an average of 31.7 sessions $(S D=23.3$, range $=5-120, n=175)$ over an average of 40.3 weeks $(S D=26$, range $=4$ $104, n=222)$.

[INSERT TABLE 9 ABOUT HERE]

[INSERT TABLE 10 ABOUT HERE]

\section{Discussion of Study 2}

This study aimed to explore the clinical services delivered by SLTs in Australia. Similar to previous surveys of clinical practice in Australia and internationally, we found that SLTs report using a range of intervention approaches which have varying levels of external evidence supporting their efficacy (e.g., McLeod and Baker, 2014, Joffe and Pring, 2008). Congruent with the findings from Study 1, above, this survey found that the most common service delivery model used by SLTs in Australia is individual intervention (96.4\%), delivered by an SLT 
(97.8\%). However, we found that SLTs in Australia reported that a wider range of people directly deliver intervention to children with phonology-based SSD than is found in the external evidence base: for example, parents and teachers were commonly reported to be involved in directly delivering intervention services to children with SSD in Australia. The results of the survey indicate that SLTs report delivering intervention in a range of locations, including schools (48.7\%), private clinics (38.0\%) and community health or hospital settings (31.9\%). This mirrors findings from other international surveys of clinical practice, which have identified that services for children with SSD are commonly delivered in preschools, early childhood centres, schools and homes (Brumbaugh and Smit, 2013). Although delivering services in these locations was not commonplace in the evidence base, the propensity for intervention research to be delivered in university clinics likely reflects that most intervention research has been carried out by academic-researchers. This point aside, the results of the survey indicated that the most common service delivery models used by SLTs in Australia broadly align with the external evidence base for phonological interventions. However, this was not the case for the intensity of these services. When comparing the intensity of intervention reported to be delivered by SLTs in Australia with the evidence base, some stark differences are apparent. Australian SLTs most commonly deliver intervention $1 \times$ week $(62.3 \%)$ in sessions lasting $30-44$ minutes $(62.4 \%)$, indicating a lower dose frequency and session duration than found in the external evidence base. Further, SLTs in Australia reported providing fewer intervention sessions (an average of 22.7 compared to 43.8) over a shorter total intervention duration (an average of 38.2 weeks compared to 61.3) than is presented within the evidence base for discharge from services. Although most SLTs working in Australia reported meeting or exceeding the recommended 50-99 production trials per session (51.9\% of SLTs), many (44.4\%) fell short of this benchmark. A striking finding 
was SLTs' preference for delivering production, rather than perception or conceptual, trials within a session. Such a preference for intervention involving the production of speech targets mirrors the emphasis on production found in many intervention approaches within the extant literature.

While some studies included in Study 1 were published after the survey was conducted ( $n$ $=10$ ), this more recent external evidence provided no new insights or information regarding intervention intensity for phonological interventions. Interestingly, SLTs in Australia reported that ideally they would deliver services more frequently, in longer sessions to children with SSD. In fact, the ideal dose frequency and session duration reported by respondents broadly align with the external evidence base, suggesting that Australian SLTs are aware of, but limited in applying, external evidence to their clinical practice.

The intensity of services reported to be delivered by SLTs in Australia is similar to that reported by Glogowska et al. (2000) in their RCT comparing "watchful waiting" with community-based speech and language therapy services. This RCT provided little evidence for the effectiveness of services delivered at this low intensity, raising concerns for the effectiveness of services being provided to many children in Australia. The findings of this survey parallel results from other international surveys of SLTs' intervention intensity when working with children with SSD, which have also shown that services are often delivered at intensities below those commonly found in the evidence base (e.g., To et al., 2012, Brumbaugh and Smit, 2013). This raises the questions: why is there a mismatch in intervention intensity, and what strategies could be used to overcome this mismatch? These questions will be considered below.

\section{General Discussion}


In this paper, we presented two studies examining the issues of service delivery and intervention intensity for phonology-based SSD. The first study was an updated review of the external evidence base for phonological interventions, which presented detailed information about the intensity of intervention typically delivered in empirical research. The second study-a survey of Australian SLTs' clinical practice — showed that children with phonology-based SSD in Australia may not be receiving evidence-based intensities of intervention: a concerning finding, given the importance of implementing interventions as described, with the same service delivery models and at equivalent intensities, within the published external evidence (e.g., Kaderavek and Justice, 2010). By considering these two sources of evidence—empirical evidence and evidence from day-to-day clinical practice — we offer unique insights into the challenges of engaging in evidence-based practice.

Barriers such as time, access to research evidence, and training are often cited as limiting the application of external evidence to clinical practice (Hoffman et al., 2013, O'Connor and Pettigrew, 2009). Other barriers include the evidence base itself: although this paper presented a review of intervention intensity reported within the extant literature, the optimal intensity of intervention needed to remediate phonology-based SSD from the point of referral to discharge remains unknown (Baker, 2012). In addition, much of the research evidence for intervention within the field of speech and language therapy includes insufficient detail to allow for faithful replication and implementation into clinical practice (Ludemann et al., 2017). These barriers speak to the challenges and complexities of conducting evidence-based practice within the realities of everyday practice (Kamhi, 2006). The steps of accessing the external evidence, appraising its quality, implementing it faithfully and evaluating the outcome to answer a clinical 
question are appropriate for an ideal world, but may ignore the real-world context in which SLTs work. Strategies to support SLTs apply empirical evidence into their clinical practice are needed. Solutions that generate evidence, while considering these day-to-day realities of clinical practice, are essential. One option championed by researchers is for clinicians to generate practice-based evidence (Lof, 2011). This involves clinicians documenting evidence about treatment - including service delivery, intensity, outcomes and modifications - from within their own clinical practice. Such evidence would capture the complexities of clinical caseloads within local contexts and be "highly clinically relevant" (Ebbels, 2017: 218). This evidence would also inform SLTs about the effectiveness of their clinical practice for children with SSD, and could demonstrate that their intervention is having a positive outcome for the children on their caseload or lead to appropriate modifications. One example of this type of evidence within the field of SSD was presented by Skelton and Richard (2016), in which the everyday school-based group intervention services for children with articulation disorders were evaluated.

Another solution could be for researchers, when designing and conducting clinical research, to consider these broader issues facing SLTs who are attempting to apply external evidence into their clinical practice. For example, researchers could consider the local contexts in which interventions are delivered to create evidence that is directly applicable to clinical practice and thus potentially easier for SLTs to implement. Another example would be to investigate the effectiveness of strategies commonly used by SLTs to overcome service delivery barriers: one such strategy frequently used by SLTs in Australia and Canada is to train parents to deliver intervention (Lim et al., 2017). In light of the findings from a review cautioning the use of this strategy to overcome service delivery barriers, research investigating this model of service delivery would be welcome (Tosh et al., 2017). Investigations considering the local context 
would address concerns raised by SLTs that research evidence lacks clinical utility (Foster et al., 2015). Such clinically-relevant research, developed through partnerships between clinicians and researchers (Ebbels, 2017), could support SLTs as they strive to deliver evidence-based services to the children on their caseloads.

\section{Limitations}

The papers included in Study 1-all reporting to investigate a phonological intervention or treatment or therapy - were identified through a comprehensive search using broad search terms. Many of these studies - 31.6\% — reported to include at least one participant with a concomitant disorder such as a language disorder, childhood apraxia of speech, or hearing impairment. It may be that the phonological intervention delivered to these children was influenced by the presence of these concomitant disorders, potentially limiting the interpretation of the results specific to phonology-based SSD. Conversely, the inclusion of these studies may increase the clinical utility of the findings, as children with SSD on SLTs' caseloads often present with concomitant disorders (Broomfield and Dodd, 2004a).

Due to the range of research designs included in Study 1, an appraisal of the quality of the studies was not completed. Such a decision reflected the need for a variety of quality assessment tools to properly assess each research design. Given that different quality assessment tools each comprise different items, arriving at meaningful comparisons and conclusions about the quality of each study would be difficult.

The primary limitation of Study 2 is the self-reported nature of the findings. While this limitation is inherent to most survey research, future research could consider using a wider range of research methods to investigate SLTs' clinical practice. This could include, for example, file audits or direct observation of SLTs working with children with phonology-based SSD. The use 


\begin{abstract}
of such methods may overcome the dual issues of self-selection bias and social desirability response bias in survey research (Podsakoff et al., 2012).
\end{abstract}

\title{
Conclusions and Directions for the Future
}

The first study presented in this paper reviewed the external evidence base for phonological interventions, with a particular focus on service delivery and intervention intensity. Intervention within the evidence base has predominately been delivered 2-3 times per week by an SLT, in individual sessions lasting 30-60 minutes comprising at least 50 to 100 production trials. While providing some guidance on service delivery and intervention intensity, the findings of Study 1 emphasise the need for more detailed reporting of intervention intensity within published research and further investigations of optimal intervention intensity for SSD, particularly regarding cumulative intervention intensity and the total duration of intervention needed to discharge a child with intelligible speech. The second study presented in this paper demonstrated that this intensity of intervention is infrequently being delivered in clinical practice in Australia. While it appears that SLTs in Australia are aware of the external evidence for dose frequency and session duration, workplace factors limit its application to intervention services. This gap between the external evidence and clinical practice is not unique to Australia: surveys of SLTs from the US and Hong Kong have shown that many SLTs are delivering services to children with SSD at lower intensities than that found in the evidence base (Brumbaugh and Smit, 2013, To et al., 2012). Although workplace and caseload barriers may influence SLTs' clinical decisions about intervention intensity, other factors - such as limited details in reporting - may hinder the application of research evidence to clinical practice.

The differences and the range of service delivery models, intervention approaches and intensities found in these two studies of the external and internal evidence bases raise challenges 
for researchers aiming to determine the effectiveness of interventions within a real-world context (Brumbaugh and Smit, 2013). This reinforces the need for the widespread creation and dissemination of practice-based evidence (Ebbels, 2017, Lof, 2011), in which intervention effectiveness can be determined within the realities of clinical practice. Future research needs to consider these realities while simultaneously aspiring to match the empirical recommendations regarding intervention intensity. Generating empirical evidence for innovative solutions to the service delivery restrictions experienced worldwide would provide SLTs with evidence that is relevant and directly applicable to clinical practice.

\section{Acknowledgements}

The authors would like to thank XXX for assistance with data entry for a previous version of the spreadsheet that was used in Study 1. We would also like to thank the SLTs who completed the questionnaire in Study 2, the organisations who helped distribute the questionnaire. and XXX, XXX, XXX, XXX and XXX for their useful comments when piloting the questionnaire.

\section{Declarations}

The authors report no conflicts of interest. The authors alone are responsible for the content and writing of the paper. 


\section{References}

ALLEN, M. M. 2013, Intervention efficacy and intensity for children with speech sound disorder. Journal of Speech, Language, and Hearing Research, 56, 865-877.

AMERICAN SPEECH-LANGUAGE-HEARING ASSOCIATION. 2004, Evidence-based practice in communication disorders: An introduction [Online]. Available: www.asha.org/policy [Accessed].

AMERICAN SPEECH-LANGUAGE-HEARING ASSOCIATION 2011, National Outcomes Measurement System: Pre-Kindergarten National Data Report 2011, Rockville, MD, National Center for Evidence-Based Practice in Communication Disorders.

BAKER, E. 2012, Optimal intervention intensity. International Journal of Speech-Language Pathology, 14, 401-409.

BAKER, E. and MCLEOD, S. 2011, Evidence-based practice for children with speech sound disorders: Part 1 narrative review. Language, Speech, and Hearing Services in Schools, $42,102-139$.

BERRY, M. D. and EISENSON, J. 1956, Speech Disorders: Principals and Practices of Therapy, New York, NY, Appleton Century Crofts.

BOWEN, C. and CUPPLES, L. 1999, Parents and children together (PACT): A collaborative approach to phonological therapy. International Journal of Language and Communication Disorders, 34, 35-55.

BRANDEL, J. and LOEB, D. F. 2011, Program intensity and service delivery models in the schools: SLP survey results. Language, Speech, and Hearing Services in Schools, 42, 461-490. 
BROOMFIELD, J. and DODD, B. 2004a, Children with speech and language disability: caseload characteristics. International Journal of Language and Communication Disorders, 39, 303-324.

BROOMFIELD, J. and DODD, B. 2004b, The nature of referred subtypes of primary speech disability. Child Language Teaching and Therapy, 20, 135-151.

BRUMBAUGH, K. M. and SMIT, A. B. 2013, Treating children ages 3-6 who have speech sound disorder: A survey. Language, Speech, and Hearing Services in Schools, 44, 306319.

DODD, B. and BARKER, R. 1990, The efficacy of utilizing parents and teachers as agents of therapy for children with phonological disorders. Australian Journal of Human Communication Disorders, 18, 29-44.

DODD, B. and BRADFORD, A. 2000, A comparison of three therapy models for children with different types of developmental phonological disorder. International Journal of Language and Communication Disorders, 35, 189-209.

DOLlagHAN, C. A. 2007, The Handbook for Evidence Based Practice in Communication Disorders, Baltimore, MD, Paul H. Brookes.

DUNN, C. and BARRON, C. 1982, A treatment program for disordered phonology: Phonetic and linguistic considerations. Language, Speech, and Hearing Services in Schools, 13, 100-109.

EBBELS, S. H. 2017, Intervention research: Appraising study designs, interpreting findings and creating research in clinical practice. International Journal of Speech-Language Pathology, 19, 218-231. 
FOSTER, A., WORRALL, L., ROSE, M. and O'HALLORAN, R. 2015, 'That doesn't translate': The role of evidence-based practice in disempowering speech pathologists in acute aphasia management. International Journal of Language and Communication Disorders, $50,547-563$.

GIERUT, J. A. 1990, Differential learning of phonological oppositions. Journal of Speech and Hearing Research, 33, 540-549.

GILDERSLEEVE-NEUMANN, C. and GOLDSTEIN, B. A. 2015, Cross-linguistic generalization in the treatment of two sequential Spanish-English bilingual children with speech sound disorders. International Journal of Speech-Language Pathology, 17, $26-40$.

GILLON, G. T. 2000, The efficacy of phonological awareness intervention for children with spoken language impairment. Language, Speech, and Hearing Services in Schools, 31, $126-141$.

GLOGOWSKA, M., ROULSTONE, S., ENDERBY, P. and PETERS, T. J. 2000, Randomised controlled trial of community based speech and language therapy in preschool children. British Medical Journal, 321, 1-5.

GRANT, M.J. and BOOTH, A. 2009, A typology of reviews: An analysis of 14 review types and associated methodologies. Health Information and Libraries Journal, 26, 91-108.

HAYDEN, D. 2006, The PROMPT model: Use and application for children with mixed phonological-motor impairment. International Journal of Speech-Language Pathology, 8, 265-281.

HODSON, B. and PADEN, E. P. 1983, Basic remediation concepts and procedures. Targeting intelligible speech: A phonological approach to remediation., San Diego, College-Hill Press. 
HODSON, B. W. and PADEN, E. P. 1991, Targeting Intelligible Speech: A Phonological Approach to Remediation, Austin, TX, Pro-Ed.

HOFFMAN, L. M., IRELAND, M., HALL-MILLS, S. and FLYNN, P. 2013, Evidence-based speech-language pathology practices in schools: Findings from a national survey. Language, Speech, and Hearing Services in Schools, 44, 266-280.

HOFFMAN, P. R., NORRIS, J. A. and MONJURE, J. 1990, Comparison of process targeting and whole language treatments for phonologically delayed preschool children. Language, Speech, and Hearing Services in Schools, 21, 102-109.

HOFFMANN, T. C., GLASZIOU, P. P., BOUTRON, I., MILNE, R., PERERA, R., MOHER, D., ALTMAN, D. G., BARBOUR, V., MACDONALD, H., JOHNSTON, M., LAMB, S. E., DIXON-WOODS, M., MCCULLOCH, P., WYATT, J. C., CHAN, A.-W. and MICHIE, S. 2014, Better reporting of interventions: template for intervention description and replication (TIDieR) checklist and guide. BMJ, 348.

HOWELL, J. and DEAN, E. C. 1994, Treating Phonological Disorders in Children: Metaphon: Theory to Practice, London, Whurr.

INTERNATIONAL EXPERT PANEL ON MULTILINGUAL CHILDREN'S SPEECH 2012, Multilingual children with speech sound disorders: Position paper. Bathurst, NSW, Australia: Research Institute for Professional Practice, Learning \& Education (RIPPLE), Charles Sturt University.

JARVIS, J. 1989. Taking a Metaphon approach to phonological development: A case study. Child Language Teaching and Therapy, 5, 16-32.

JOFFE, V. and PRING, T. 2008, Children with phonological problems: A survey of clinical practice. International Journal of Language and Communication Disorders, 43, 154-164. 
KADERAVEK, J. N. and JUSTICE, L. M. 2010, Fidelity: An essential component of evidencebased practice in speech-language pathology. American Journal of Speech-Language Pathology, 19, 369-379.

KAIPA, R. and PETERSON, A. M. 2016, A systematic review of treatment intensity in speech disorders. International Journal of Speech-Language Pathology, 18, 507-520.

KAMHI, A. G. 2006, Treatment decisions for children with speech-sound disorders. Language, Speech, and Hearing Services in Schools, 37, 271-279.

KLEIN, E. S. 1996, Phonological/traditional approaches to articulation therapy: A retrospective group comparison. Language, Speech, and Hearing Services in Schools, 27, 314-323.

LANCASTER, G., KEUSCH, S., LEVIN, A., PRING, T. and MARTIN, S. 2010, Treating children with phonological problems: Does an eclectic approach to therapy work? International Journal of Language and Communication Disorders, 45, 174-181.

LANCASTER, G. and POPE, L. 1989, Working with children's phonology, Oxon, UK, Winslow Press.

LAW, J., GARRETT, Z. and NYE, C. 2004, The efficacy of treatment for children with developmental speech and language delay/disorder: A meta-analysis. Journal of Speech, Language, and Hearing Research, 47, 924-943.

LEWIS, B. A., PATTON, E., FREEBAIRN, L., TAG, J., IYENGAR, S. K., STEIN, C. M. and TAYLOR, H. G. 2016, Psychosocial co-morbidities in adolescents and adults with histories of communication disorders. Journal of Communication Disorders, 61, 60-70.

LIM, J., MCCABE, P. and PURCELL, A. 2017, Challenges and solutions in speech-language pathology service delivery across Australia and Canada. European Journal for Person Centered Healthcare, 5, 120-128. 
LOF, G. L. 2011, Science-based practice and the speech-language pathologist. International Journal of Speech-Language Pathology, 13, 189-196.

LUDEMANN, A., POWER, E. and HOFFMANN, T. C. 2017, Investigating the adequacy of intervention descriptions in recent speech-language pathology literature: Is evidence from randomized trials useable? American Journal of Speech-Language Pathology, 1-13.

MCCORMACK, J., HARRISON, L. J., MCLEOD, S. and MCALLISTER, L. 2011, A nationally representative study of the association between communication impairment at 4-5 years and children's life activities at 7-9 years. Journal of Speech, Language, and Hearing Research, 54, 1328-1348.

MCCURTIN, A. and CLIFFORD, A. M. 2015, What are the primary influences on treatment decisions? How does this reflect on evidence-based practice? Indications from the discipline of speech and language therapy. Journal of Evaluation in Clinical Practice, 21, 1178-1189.

MCLEOD, S. and BAKER, E. Current clinical practice for children with speech impairment. In: MURDOCH, B. E., GOOZEE, J., WHELAN, B. M. \& DOCKING, K., eds. Proceedings of the 26th world congress of the International Association of Logopedics and Phoniatrics, 2004 Brisbane. University of Queensland.

MCLEOD, S. and BAKER, E. 2014, Speech-language pathologists' practices regarding assessment, analysis, target selection, intervention, and service delivery for children with speech sound disorders. Clinical Linguistics and Phonetics, 28, 508-531.

MCLEOD, S., PRESS, F. and PHELAN, C. 2010, The (in)visibility of children with communication impairment in Australian health, education, and disability legislation and policies. Asia Pacific Journal of Speech, Language and Hearing, 13, 67-75. 
MONTGOMERY, J. K. and BONDERMAN, R. 1989, Serving preschool children with severe phonological disorders. Language, Speech, and Hearing Services in Schools, 20, 76-84.

MOTA, H. B., KESKE-SOARES, M., BAGETTI, T., CERON, M. I. and FILHA, M. 2007, Análise comparativa da eficiência de três diferentes modelos de terapia fonológica [Comparative analyses of the effectiveness of three different phonological therapy models]. Pró-Fono Revista de Atualização Científica, 19, 67-74.

NUFFIELD HEARING AND SPEECH CENTRE 2004, The Nuffield Centre Dyspraxia Programme, London, UK, Nuffield Centre Dyspraxia Programme Ltd.

O'CONNOR, S. and PETTIGREW, C. M. 2009, The barriers perceived to prevent the successful implementation of evidence-based practice by speech and language therapists. International Journal of Language and Communication Disorders, 44, 1018-1035.

ODOM, S. L. 2009, The tie that binds: Evidence-based practice, implementation science, and outcomes for children. Topics in Early Childhood Special Education, 29, 53-61.

PAMPLONA, M. C., YSUNZA, A. and MORALES, S. 2014, Strategies for treating compensatory articulation in patients with cleft palate. International Journal of Biomedical Science, 10, 43-51.

PASSEY, J. 1990, Cued Articulation, Melbourne, Australia, Australian Council for Educational Research.

PENNEY, G., FEE, J. and DOWDLE, C. 1994, Vowel assessment and remediation: A case study. Child Language Teaching and Therapy, 10, 47-66.

PODSAKOFF, P.M., MACKENZIE, S.B., and PODSAKOFF, N.P. 2012, Sources of method bias in social science research and recommendations on how to control it. Annual Review of Psychology, 63, 539-569. 
PRING, T., FLOOD, E., DODD, B. and JOFFE, V. 2012, The working practices and clinical experiences of paediatric speech and language therapists: a national UK survey. International Journal of Language and Communication Disorders, 47, 696-708.

ROYAL COLLEGE OF SPEECH \& LANGUAGE THERAPISTS 2016, Guidance for Speech and Language Therapists on their roles and responsibilities under the Children and Families Act 2014 and associated Code of Practice. Royal College of Speech and Language Therapists Position Paper. London: Royal College of Speech and Language Therapists.

RUGGERO, L., MCCABE, P., BALLARD, K. J. and MUNRO, N. 2012, Paediatric speechlanguage pathology service delivery: An exploratory survey of Australian parents. International Journal of Speech-Language Pathology, 14, 338-350.

SENATE COMMUNITY AFFAIRS REFERENCES COMMITTEE 2014, Prevalence of different types of speech, language and communication disorders and speech pathology services in Australia. In: COMMITTEE, S. C. A. R. (ed.). Canberra, Australia: Senate Community Affairs References Committee.

SKAHAN, S. M., WATSON, M. and LOF, G. L. 2007, Speech-language pathologists' assessment practices for children with suspected speech sound disorders: Results of a national survey. American Journal of Speech-Language Pathology, 16, 246-59.

SKELTON, S. L. and RICHARD, J. T. 2016, Application of a motor learning treatment for speech sound disorders in small groups. Perceptual and Motor Skills, 122, 840-854.

SOMMERS, R. K., LOGSDON, B. and WRIGHT, J. 1992, A review and critical analysis of treatment research related to articulation and phonological disorders. Journal of Communication Disorders, 25, 3-22. 
SPEECH PATHOLOGY AUSTRALIA 2014, Submission to the Inquiry into the Prevlance of Differnt Types of Speech, Language and Communication Disorders and Speech Pathology Services in Australia. Speech Pathology Australia.

SPEECH PATHOLOGY AUSTRALIA. 2015, Scope of Practice in Speech Pathology [Online]. [Accessed].

SUGDEN, E., BAKER, E., MUNRO, N. and WILLIAMS, A. L. 2016, Involvement of parents in intervention for childhood speech sound disorders: A review of the evidence. International Journal of Language and Communication Disorders, 51, 597-625.

TO, C. K. S., LAW, T. and CHEUNG, P. S. P. 2012, Treatment intensity in everyday clinical management of speech sound disorders in Hong Kong. International Journal of SpeechLanguage Pathology, 14, 462-466.

TOSH, R., ARNOTT, W. and SCARINCI, N. 2017, Parent-implemented home therapy programmes for speech and language: a systematic review. International Journal of Language and Communication Disorders, 52, 253-269.

VAN RIPER, C. 1939, Speech Correction Principles and Methods, New York, Prentice Hall.

VERDON, S., WILSON, L., SMITH-TAMARAY, M. and MCALLISTER, L. 2011, An investigation of equity of rural speech-language pathology services for children: A geographic perspective. International Journal of Speech-Language Pathology, 13, 239250.

WARREN, S. F., FEY, M. E. and YODER, P. J. 2007, Differential treatment intensity research: A missing link to creating optimally effective communication interventions. Mental Retardation and Developmental Disabilities Research Reviews, 13, 70-77. 
WATTS PAPPAS, N., MCLEOD, S., MCALLISTER, L. and MCKINNON, D. H. 2008, Parental involvement in speech intervention: a national survey. Clinical Linguistics and Phonetics, 22, 335-344.

WEINER, F. 1981, Treatment of phonological disability using the method of meaningful minimal contrast: two case studies. Journal of Speech and Hearing Disorders, 46, $97-$ 103.

WILLIAMS, A. L. 2000, Multiple oppositions: Theoretical foundations for an alternative contrastive intervention approach. American Journal of Speech-Language Pathology, 9, 282.

WILLIAMS, A. L. 2012, Intensity in phonological intervention: Is there a prescribed amount? International Journal of Speech-Language Pathology, 14, 456-461. 
TABLE 1. SERVICE DELIVERY MODELS USED WITHIN 206ª INTERVENTION STUDIES PUBLISHED BETWEEN 1979 AND 2016

\begin{tabular}{|c|c|c|c|c|c|}
\hline How & $n(\%)$ & Who & $n(\%)$ & Where & $n(\%)$ \\
\hline Individual & $160(75.5 \%)$ & SLT $^{b}$ & $184(86.8 \%)$ & University & $116(54.7 \%)$ \\
\hline Group & $17(8.0 \%)$ & SLT and parent & $10(4.7 \%)$ & School/preschool & $19(9.0 \%)$ \\
\hline Individual and group & $17(8.0 \%)$ & Experimenter & $5(2.4 \%)$ & Hospital & $15(7.1 \%)$ \\
\hline Group parent training & $4(1.9 \%)$ & Parent & $4(1.9 \%)$ & Community clinic or health centre & $14(6.6 \%)$ \\
\hline Group teacher training & $1(0.5 \%)$ & SLT and/or speech aide & $4(1.9 \%)$ & University or preschool & $5(2.4 \%)$ \\
\hline Individual OR group & $1(0.5 \%)$ & Teacher or specialist teacher & $3(1.4 \%)$ & University and home & $4(1.9 \%)$ \\
\hline \multirow[t]{10}{*}{ Not reported } & $12(5.7 \%)$ & Non-SLT student & $1(0.5 \%)$ & Private clinic & $3(1.4 \%)$ \\
\hline & & Research assistant or parent & $1(0.5 \%)$ & Home & $3(1.4 \%)$ \\
\hline & & & & Home and school & $2(0.9 \%)$ \\
\hline & & & & $\begin{array}{l}\text { Home and early intervention } \\
\text { centre }\end{array}$ & $2(0.9 \%)$ \\
\hline & & & & Community clinic and home & $2(0.9 \%)$ \\
\hline & & & & School or home & $2(0.9 \%)$ \\
\hline & & & & University or community clinic & $1(0.5 \%)$ \\
\hline & & & & University and school & $1(0.5 \%)$ \\
\hline & & & & Hospital and home & $1(0.5 \%)$ \\
\hline & & & & Not reported & $22(10.4 \%)$ \\
\hline
\end{tabular}

${ }^{\mathrm{a}}$ This includes the 194 papers reporting on a specific intervention or case, which comprised 206 studies. Six of these studies included two service delivery models within their investigation, which were coded separately, resulting in 212 service delivery models investigated within the literature.

${ }^{\mathrm{b}} \mathrm{SLT}=$ speech-language therapist. This includes studies reporting that SLT students delivered intervention services. 
TABLE 2. DOSE FREQUENCY AND SESSION DURATION PROVIDED IN $206^{\mathrm{a}}$ PHONOLOGICAL INTERVENTION STUDIES

\begin{tabular}{|c|c|c|c|}
\hline Dose frequency & $n(\%)$ & Session duration & $n(\%)$ \\
\hline Less than $1 \times$ month & $1(0.5 \%)$ & Less than 30 minutes & $7(3.3 \%)$ \\
\hline $1 \times$ month & $1(0.5 \%)$ & 30 minutes & $38(17.9 \%)$ \\
\hline $2 \times$ month & $3(1.4 \%)$ & 45 minutes & $34(16.0 \%)$ \\
\hline $1 \times$ week & $41(19.3 \%)$ & 50 minutes & $16(7.5 \%)$ \\
\hline $2 \times$ week & $71(33.5 \%)$ & 60 minutes & $42(19.8 \%)$ \\
\hline $3 \times$ week & $40(18.9 \%)$ & More than 60 minutes & $13(6.1 \%)$ \\
\hline More than $3 \times$ week & $9(4.2 \%)$ & Other & $12(5.7 \%)$ \\
\hline Other & $8(3.8 \%)$ & Combination $^{\mathrm{b}}$ & $17(8.0 \%)$ \\
\hline Combination $^{\mathrm{b}}$ & $8(3.8 \%)$ & Not reported & $33(15.6 \%)$ \\
\hline Unclear & $2(0.9 \%)$ & & \\
\hline Not reported & $28(13.2 \%)$ & & \\
\hline \multicolumn{4}{|c|}{$\begin{array}{l}\text { a The numbers total } 212 \text { as six studies explicitly reported different dose frequencies and } \\
\text { session duration for each of the groups in the study } \\
\text { b Studies may have included more than one duration and/or frequency for a participant, } \\
\text { group or intervention (e.g. in the case study presented in Jarvis, 1989, intervention was } \\
\text { delivered } 3 \times \text { per week, then } 2 \times \text { per week, then } 1 \times \text { per week) }\end{array}$} \\
\hline
\end{tabular}


TABLE 3. DOSE PROVIDED PER SESSION ACROSS $206^{\mathrm{a}}$ PHONOLOGICAL INTERVENTION STUDIES

\begin{tabular}{|c|c|c|c|}
\hline & $\begin{array}{c}\text { Production } \\
\text { dose }\end{array}$ & $\begin{array}{l}\text { Perception } \\
\text { dose }\end{array}$ & $\begin{array}{c}\text { Conceptual } \\
\text { dose }\end{array}$ \\
\hline Total studies reporting information $n(\%)$ & $47(21.6 \%)$ & $10(4.6 \%)$ & 0 \\
\hline Total studies with unclear reporting $n(\%)$ & $3(1.4 \%)$ & $3(1.4 \%)$ & 0 \\
\hline Total studies reporting no information $n(\%)$ & $168(77.1 \%)$ & $205(94.0 \%)$ & $\begin{array}{c}218 \\
(100.0 \%)\end{array}$ \\
\hline Minimum dose per session & 23 & 10 & - \\
\hline Maximum dose per session & 200 & 120 & - \\
\hline Average dose per session & 77.0 & 51.5 & - \\
\hline \multicolumn{4}{|c|}{$\begin{array}{l}\text { "Numbers total } 212 \text { as six studies included groups that each received different intensities of } \\
\text { intervention; these groups were coded separately. } \\
\text { Note: The number of studies "reporting no information" about dose includes studies that } \\
\text { may or may not have reported to include a specific type of dose within the intervention } \\
\text { approach (for example, although not all studies included conceptual intervention, they were } \\
\text { coded as not reported). }\end{array}$} \\
\hline
\end{tabular}


TABLE 4. DEMOGRAPHIC INFORMATION OF AUSTRALIAN SLTS

\begin{tabular}{|c|c|}
\hline Demographic & $n(\%)$ \\
\hline \multicolumn{2}{|l|}{ State/Territory of work ${ }^{a}$} \\
\hline Australian Capital Territory & $5(1.8 \%)$ \\
\hline New South Wales & $85(29.9 \%)$ \\
\hline Northern Territory & $4(1.4 \%)$ \\
\hline Queensland & $120(42.3 \%)$ \\
\hline South Australia & $8(2.8 \%)$ \\
\hline Tasmania & $4(1.4 \%)$ \\
\hline Victoria & $41(14.4 \%)$ \\
\hline Western Australia & $17(6.0 \%)$ \\
\hline \multicolumn{2}{|l|}{ Years practising as an $S L T^{b}$} \\
\hline Less than 1 year & $32(11.3 \%)$ \\
\hline Between 1 and 3 years & $61(21.6 \%)$ \\
\hline Between 4 and 6 years & $63(22.3 \%)$ \\
\hline Between 7 and 10 years & $41(14.5 \%)$ \\
\hline More than 10 years & $85(30.1 \%)$ \\
\hline \multicolumn{2}{|l|}{ Urban or regional location $^{a}$} \\
\hline Capital city (e.g., Sydney, Melbourne) & $163(57.4 \%)$ \\
\hline Regional city & $70(24.6 \%)$ \\
\hline Large country town & $26(9.2 \%)$ \\
\hline Small country town & $18(6.3 \%)$ \\
\hline Other & $7(2.5 \%)$ \\
\hline \multicolumn{2}{|l|}{ Employment status $^{c}$} \\
\hline Full-time & $187(67.3 \%)$ \\
\hline Part-time & $91(32.7 \%)$ \\
\hline \multicolumn{2}{|l|}{ Gender $^{a}$} \\
\hline Female & $282(99.3 \%)$ \\
\hline Male & $2(0.7 \%)$ \\
\hline $\begin{array}{l}\text { a valid responses } n=284 \\
\text { b valid responses } n=282 \\
\text { c valid responses } n=278\end{array}$ & \\
\hline
\end{tabular}


TABLE 5. SERVICE DELIVERY REPORTED TO BE USED BY SLTS IN AUSTRALIA

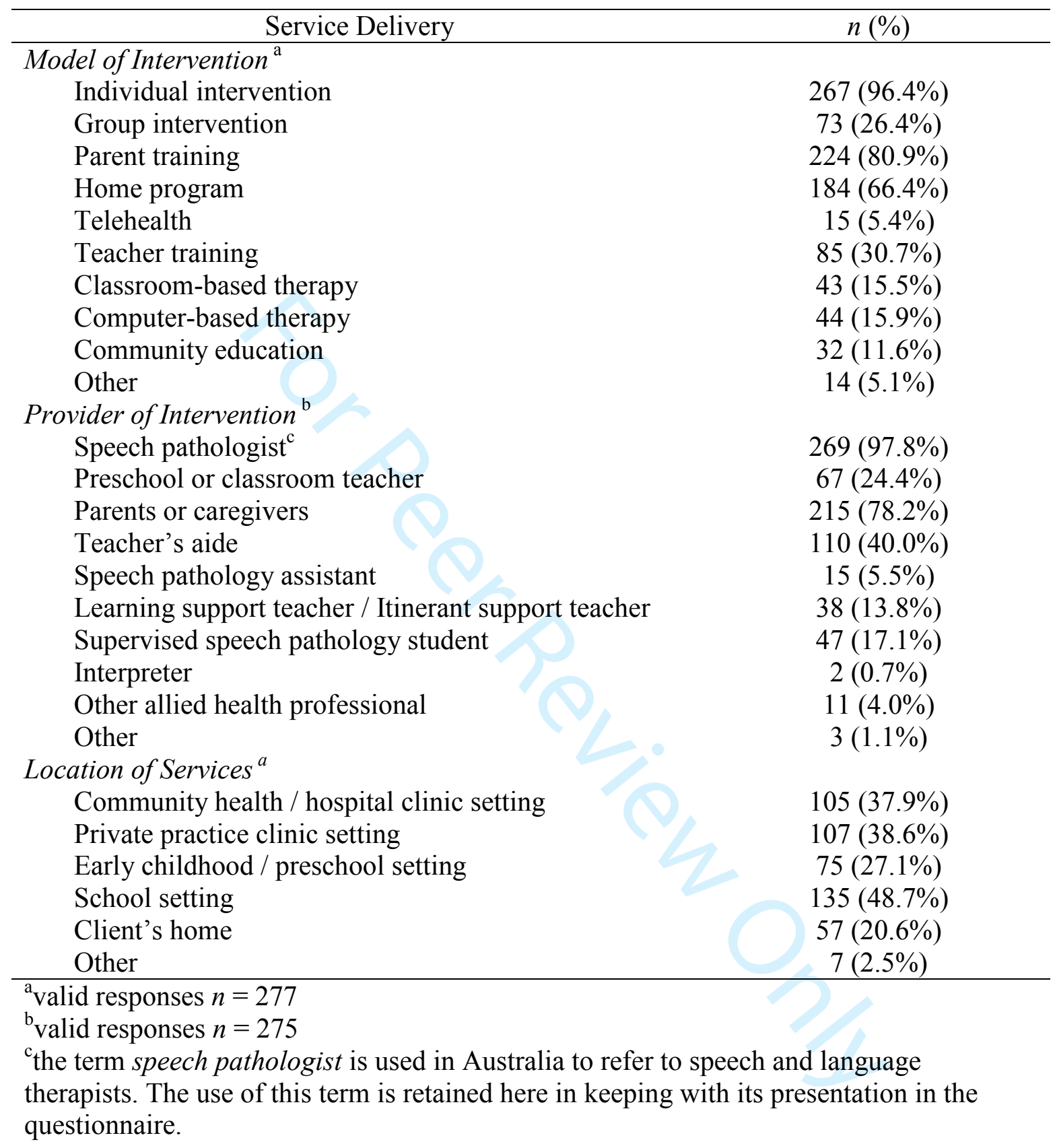


TABLE 6. INTERVENTION APPROACHES REPORTED TO BE USUALLY USED BY 270 SLTS IN AUSTRALIA

\begin{tabular}{lc}
\hline \multicolumn{1}{c}{ Intervention approach } & $n(\%)$ \\
\hline Auditory bombardment/stimulation (e.g., Hodson and Paden, 1991) & $125(46.3 \%)$ \\
Auditory discrimination (e.g., Berry and Eisenson, 1956) & $204(75.6 \%)$ \\
Core Vocabulary (e.g., Dodd and Bradford, 2000) & $127(47.0 \%)$ \\
Cued Articulation (e.g., Passey, 1990) & $152(56.3 \%)$ \\
Cycles (e.g., Hodson and Paden, 1983) & $58(21.5 \%)$ \\
Imagery approach (e.g., Klein, 1996) & $13(4.8 \%)$ \\
Maximal oppositions approach (e.g., Gierut, 1990) & $98(36.3 \%)$ \\
Metaphon (e.g., Howell and Dean, 1994) & $57(21.1 \%)$ \\
Minimal oppositions contrast (minimal pairs) (e.g., Weiner, 1981) & $224(83.0 \%)$ \\
Multiple oppositions (e.g., Williams, 2000) & $133(49.3 \%)$ \\
Non-speech oromotor intervention(e.g., Lancaster and Pope, 1989) & $5(1.9 \%)$ \\
Nuffield Centre Dyspraxia Programme (e.g., Nuffield Hearing and Speech Centre, 2004) & $94(34.8 \%)$ \\
Parents and Children Together (PACT) (e.g., Bowen and Cupples, 1999) & $22(8.1 \%)$ \\
Phonological awareness (e.g., Gillon, 2000) & $141(52.2 \%)$ \\
Prompts for Restructuring Oral Musculature Phonetic Targets (PROMPT) (e.g., Hayden, 2006) & $46(17.0 \%)$ \\
Traditional articulation therapy (e.g., Van Riper, 1939) & $174(64.4 \%)$ \\
Whole language therapy (e.g., Hoffman et al., 1990) & $30(11.1 \%)$ \\
Other & $11(4.1 \%))$ \\
\end{tabular}


TABLE 7. DOSE FREQUENCY AND SESSION DURATION REPORTED TO BE PROVIDED BY SLTS IN AUSTRALIA

\begin{tabular}{lc}
\hline Component of Intervention Intensity $^{a}$ & $n(\%)$ \\
\hline Dose frequency $^{a}$ & $3(1.1 \%)$ \\
Less than 1 session per month & $4(1.5 \%)$ \\
One session per month & $72(27.2 \%)$ \\
1 to 2 sessions per month & $165(62.3 \%)$ \\
$1 \times$ weekly & $9(3.5 \%)$ \\
$2 \times$ weekly & $9(3.4 \%)$ \\
$3 \times$ weekly & $3(1.1 \%)$ \\
More than 3 per week & \\
Session duration $b$ & $50(18.8 \%)$ \\
Less than 30 minutes & $166(62.4 \%)$ \\
30 to 44 minutes & $46(17.3 \%)$ \\
45 to 59 minutes & $0(0.0 \%)$ \\
60 to 89 minutes & $4(1.5 \%)$ \\
90 minutes or longer &
\end{tabular}

${ }^{a}$ valid responses $n=265$

${ }^{\mathrm{b}}$ valid responses $n=266$ 


\section{TABLE 8. DOSE OF PRODUCTION, PERCEPTUAL AND CONCEPTUAL TRIALS} PROVIDED BY AUSTRALIAN SLTS

\begin{tabular}{lc}
\hline Type and number of trials & $n(\%)$ \\
\hline Production $^{a}$ & \\
None & $1(0.4 \%)$ \\
Less than 20 & $18(6.8 \%)$ \\
$21-49$ & $100(37.6 \%)$ \\
$50-99$ & $106(39.8 \%)$ \\
$100-149$ & $22(8.3 \%)$ \\
$150-199$ & $8(3.0 \%)$ \\
$200+$ & $2(0.8 \%)$ \\
Unsure & $9(3.4 \%)$ \\
Perceptual $^{b}$ & \\
None & $6(2.3 \%)$ \\
Less than 20 & $129(56.2 \%)$ \\
$21-49$ & $21-49(30.6 \%)$ \\
$50-99$ & $17(6.4 \%)$ \\
$100-149$ & $2(0.8 \%)$ \\
$150-199$ & $2(0.8 \%)$ \\
$200+$ & $0(0.0 \%)$ \\
Unsure & $8(3.0 \%)$ \\
Conceptual & \\
None & \\
Less than 20 & $37(13.9 \%)$ \\
$21-49$ & $149(56.0 \%)$ \\
$50-99$ & $59(22.2 \%)$ \\
$100-149$ & $7(2.6 \%)$ \\
$150-199$ & $4(1.5 \%)$ \\
$200+$ & $0(0.0 \%)$ \\
Unsure & $0(0.0 \%)$ \\
vespos & $10(3.8 \%)$ \\
\hline
\end{tabular}

${ }^{a}$ valid responses $n=266$

${ }^{\mathrm{b}}$ valid responses $n=265$ 
TABLE 9. FACTORS INFLUENCING INTERVENTION INTENSITY REPORTED BY AUSTRALIAN SLTS

\begin{tabular}{lc}
\hline \multicolumn{1}{c}{ Factor } & $n(\%)$ \\
\hline Workplace factors $^{a}$ & $124(50.2 \%)$ \\
Waiting lists & $138(55.9 \%)$ \\
Scheduling conflicts & $95(38.5 \%)$ \\
Workplace policy & $172(69.6 \%)$ \\
Size of active caseload & $77(31.2 \%)$ \\
Funding reasons & $147(59.5 \%)$ \\
Service delivery model & $15(6.1 \%)$ \\
Other & \\
Clinician factors ${ }^{b}$ & $91(38.6 \%)$ \\
Personal factors & $94(39.8 \%)$ \\
Application of research evidence & $41(17.4 \%)$ \\
Implementing specific program & $139(58.9 \%)$ \\
Previous experience & $47(19.9 \%)$ \\
Always provided this intensity & $7(3.0 \%)$ \\
Other & \\
Client factors & \\
Funding reasons & $117(48.3 \%)$ \\
Rate of progress & $167(69.0 \%)$ \\
Family preference & $178(73.6 \%)$ \\
Severity of disorder & $176(72.7 \%)$ \\
Travel time & $66(27.3 \%)$ \\
Age of client & $122(50.4 \%)$ \\
Cultural and/or linguistic background & $51(21.1 \%)$ \\
Other & $33(13.6 \%)$ \\
\hline
\end{tabular}

\footnotetext{
${ }^{\mathrm{a}}$ valid responses $n=247$

balid responses $n=236$

c valid responses $n=242$
} 
TABLE 10. IDEAL DOSE FREQUENCY AND SESSION DURATION AS RATED BY SLTS IN AUSTRALIA

\begin{tabular}{lc}
\hline & $n(\%)$ \\
\hline Dose frequency $^{\mathrm{a}}$ & \\
Less than $1 \times$ month $_{1 \times \text { month }}$ & 0 \\
1 to 2 sessions per month & 0 \\
$1 \times$ weekly & $19(8.2 \%)$ \\
$2 \times$ weekly & $83(35.8 \%)$ \\
$3 \times$ weekly & $66(28.4 \%)$ \\
More than $3 \times$ week & $52(22.4 \%)$ \\
Other or combination & $8(3.4 \%)$ \\
Session duration & $4(1.7 \%)$ \\
Less than 30 minutes & \\
30 minutes & $22(9.5 \%)$ \\
30 to 45 minutes & $79(34.2 \%)$ \\
45 minutes & $54(23.4 \%)$ \\
45 to 60 minutes & $48(20.8 \%)$ \\
60 minutes & $12(5.2 \%)$ \\
Other & $12(5.2 \%)$ \\
\hline Vatid & $4(1.7 \%)$ \\
\hline
\end{tabular}

${ }^{\mathrm{a}}$ Valid responses $=232$

${ }^{\mathrm{b}}$ Valid responses $=231$ 


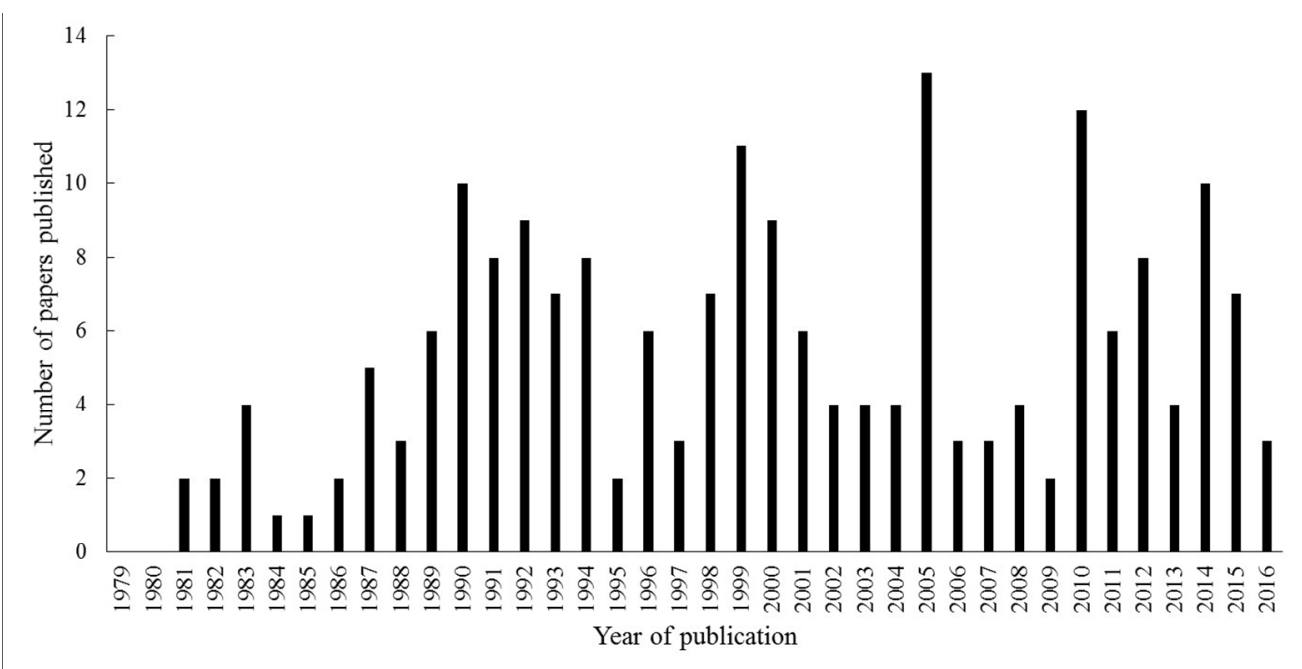

FIGURE 1. YEAR OF PUBLICATION OF 199 PHONOLOGICAL INTERVENTION PAPERS PUBLISHED BETWEEN 1979 AND 2016

$236 \times 120 \mathrm{~mm}(150 \times 150 \mathrm{DPI})$ 


\section{Appendix A: Details of service delivery and intervention intensity in phonological intervention studies published between 1979 and 2016}

This appendix provides a summary of the 199 peer-reviewed journal publications from 1979-2016 which were included in this review. Readers are encouraged to source the original publication in order to gain a greater understanding of the intervention approach, processes, and outcomes in addition to more detailed descriptions of the service delivery and intervention intensity provided in each study.

Key for research design:

$\mathrm{RCT}=$ randomised controlled trial

Non-RCT $=$ non-randomised controlled trial

SCED = single-case experimental designs that include MBD (multiple baseline design across participants or behaviours), ATD (alternating treatment design), MPD (multiple probe design), and $\mathrm{AB}$ or $\mathrm{ABA}$ (baseline $[\mathrm{A}]$ followed by treatment phase $[\mathrm{B}]$, with a possible return to baseline phase $[\mathrm{A}]$ )

Case study designs $=$ non-experimental studies involving one or more cases

\begin{tabular}{|c|c|c|c|c|c|c|}
\hline Reference & Research design & $\begin{array}{l}\text { Intervention } \\
\text { approach }\end{array}$ & $\begin{array}{c}\text { Participant } \\
\text { number and age } \\
\text { (years;months) }\end{array}$ & $\begin{array}{c}\text { Summary of service } \\
\text { delivery }^{\mathrm{a}}\end{array}$ & $\begin{array}{c}\text { Summary of intervention } \\
\text { intensity }\end{array}$ & $\begin{array}{c}\text { Reporting of } \\
\text { intervention } \\
\text { intensity }^{b}\end{array}$ \\
\hline Abraham (1993) & ATD & $\begin{array}{l}\text { Phonetic approach } \\
\text { (syllable } \\
\text { imitation) } \\
\text { compared with } \\
\text { minimal pairs }\end{array}$ & $n=4(5 ; 0-10 ; 5)$ & Individual intervention. & $\begin{array}{l}1 \times 45 \text {-min session per day } \\
\text { on consecutive school } \\
\text { days. For both } \\
\text { intervention approaches } \\
\text { approach, a production } \\
\text { dose of } 25 \text { was provided } \\
\text { (total } 50 \text { per session). }\end{array}$ & $4 / 7$ \\
\hline $\begin{array}{l}\text { Adams, Nightingale, } \\
\text { Hesketh and Hall } \\
\text { (2000) }\end{array}$ & Non-RCT & $\begin{array}{l}\text { Metaphonological } \\
\text { intervention }\end{array}$ & $\begin{array}{l}n=65 \\
\text { Experimental group, } \\
\quad n=31(3 ; 6-5 ; 0) \\
\text { Control group, } n= \\
\quad 34\left(M_{\text {age }}=4 ; 2\right)\end{array}$ & $\begin{array}{l}\text { Individual intervention } \\
\text { delivered by an SLT. }\end{array}$ & $\begin{array}{l}10 \text { sessions delivered over } \\
10 \text { weeks. }\end{array}$ & $4 / 7$ \\
\hline
\end{tabular}




\begin{tabular}{|c|c|c|c|c|c|c|}
\hline Reference & Research design & $\begin{array}{l}\text { Intervention } \\
\text { approach }\end{array}$ & $\begin{array}{c}\text { Participant } \\
\text { number and age } \\
\text { (years;months) }\end{array}$ & $\begin{array}{c}\text { Summary of service } \\
\text { delivery }^{\mathrm{a}}\end{array}$ & $\begin{array}{c}\text { Summary of intervention } \\
\text { intensity }^{\mathrm{a}}\end{array}$ & $\begin{array}{l}\text { Reporting of } \\
\text { intervention } \\
\text { intensity }^{b}\end{array}$ \\
\hline Allen (2013) & RCT & Multiple oppositions & $\begin{array}{l}n=54 \\
\text { Experimental group } \\
\quad(\mathrm{P} 1), n=19\left(M_{\text {age }}\right. \\
\quad=52.8 \text { months }) \\
\text { Experimental group } \\
\quad(\mathrm{P} 3), n=19\left(M_{\text {age }}\right. \\
\quad=50.6 \text { months }) \\
\text { Active control group } \\
\quad(\mathrm{C}), n=16\left(M_{\text {age }}\right. \\
\quad=51.9 \text { months })\end{array}$ & $\begin{array}{l}\text { For all groups, intervention } \\
\text { was delivered either } \\
\text { singly or in a pair by an } \\
\text { SLT or SLT-assistant. } \\
\text { Intervention was } \\
\text { delivered in } \\
\text { developmental } \\
\text { preschools, preschools, } \\
\text { childcare, or home. }\end{array}$ & $\begin{array}{l}\text { P1 condition: } 1 \times 30-\text { min } \\
\text { session weekly for } 24 \\
\text { weeks (total } 24 \\
\text { sessions), with a } \\
\text { minimum dose of } 81 \\
\text { production trials per } \\
\text { session (cumulative } \\
\text { intervention intensity } \\
\text { 1,944). } \\
\text { P3 condition: } 3 \times 30 \text {-min } \\
\text { session weekly for } 8 \\
\text { weeks (total } 24 \\
\text { sessions), with a } \\
\text { minimum dose of } 81 \\
\text { production trials per } \\
\text { session (cumulative } \\
\text { intervention intensity } \\
\text { 1,944). }\end{array}$ & $7 / 7$ \\
\hline $\begin{array}{l}\text { Almost and Rosenbaum } \\
\text { (1998) }\end{array}$ & $\mathrm{RCT}$ & $\begin{array}{l}\text { Modified cycles } \\
\text { including minimal } \\
\text { pairs }\end{array}$ & $n=26(2 ; 9-5 ; 1)$ & $\begin{array}{l}\text { Individual intervention, } \\
\text { delivered by an SLT in } \\
\text { the SLT department of a } \\
\text { hospital. }\end{array}$ & $\begin{array}{l}2 \times 30 \text {-min sessions per } \\
\text { week over a } 4 \text { month } \\
\text { block. Participants } \\
\text { received between } 14 \\
\text { and } 29 \text { sessions in total. }\end{array}$ & $5 / 7$ \\
\hline $\begin{array}{l}\text { Bagetti, Ceron, Mota and } \\
\text { Keske-Soares (2012) }\end{array}$ & $\begin{array}{l}\text { Between groups } \\
\text { design }\end{array}$ & $\begin{array}{l}\text { Modified maximal } \\
\text { oppositions }\end{array}$ & $n=7(3 ; 10-6 ; 9)$ & $\begin{array}{l}\text { Delivered in a university } \\
\text { clinic by an SLT. }\end{array}$ & $\begin{array}{l}20 \text { sessions for six of the } \\
\text { participants, with one } \\
\text { participant discharged } \\
\text { after } 10 \text { sessions. }\end{array}$ & $2 / 7$ \\
\hline
\end{tabular}




\begin{tabular}{|c|c|c|c|c|c|c|}
\hline Reference & Research design & $\begin{array}{l}\text { Intervention } \\
\text { approach }\end{array}$ & $\begin{array}{c}\text { Participant } \\
\text { number and age } \\
\text { (years;months) }\end{array}$ & $\begin{array}{c}\text { Summary of service } \\
\text { delivery }^{\mathbf{a}}\end{array}$ & $\begin{array}{c}\text { Summary of intervention } \\
\text { intensity }\end{array}$ & $\begin{array}{c}\text { Reporting of } \\
\text { intervention } \\
\text { intensity }\end{array}$ \\
\hline $\begin{array}{l}\text { Baker and McLeod } \\
\text { (2004) }\end{array}$ & $\begin{array}{l}\mathrm{AB} \text { with control } \\
\text { behaviour }\end{array}$ & Minimal pairs & $\begin{array}{c}n=2, \text { 'James' }(4 ; 4), \\
\text { 'Cody' }(4 ; 9)\end{array}$ & $\begin{array}{l}\text { Individual intervention, } \\
\text { delivered by an SLT in } \\
\text { a university clinic. }\end{array}$ & $\begin{array}{l}2 \times 45 \text {-min sessions per } \\
\text { week. Each session } \\
\text { comprised } 100 \\
\text { production trials. Cody } \\
\text { acquired the speech } \\
\text { target within } 7 \text { weeks } \\
\text { (12 sessions) of } \\
\text { intervention, whereas } \\
\text { James required } 5 \\
\text { months (32 sessions) of } \\
\text { intervention. }\end{array}$ & $6 / 7$ \\
\hline $\begin{array}{l}\text { Baker and McLeod } \\
\text { (2011) }\end{array}$ & Narrative review & $\begin{array}{l}\text { A range of } \\
\text { phonological and } \\
\text { articulation-based } \\
\text { approaches }\end{array}$ & $\begin{array}{l}134 \text { articles } \\
\text { spanning } 40 \text { years }\end{array}$ & $\begin{array}{l}\text { Varied across included } \\
\text { studies. }\end{array}$ & $\begin{array}{l}\text { Varied across included } \\
\text { studies. }\end{array}$ & $-c$ \\
\hline $\begin{array}{l}\text { Barberena, Keske- } \\
\text { Soares, Cervi and } \\
\text { Brandão (2014) }\end{array}$ & $\begin{array}{l}\text { Between groups } \\
\text { design }\end{array}$ & $\begin{array}{l}\text { ABAB withdrawal } \\
\text { and multiple } \\
\text { probes }\end{array}$ & $n=8\left(\mathrm{M}_{\mathrm{age}}=5 ; 5\right)$ & $\begin{array}{l}\text { Delivered in a university } \\
\text { clinic by an SLT. }\end{array}$ & $\begin{array}{l}2 \times 45 \text {-min sessions per } \\
\text { week for } 5 \text { weeks. }\end{array}$ & $4 / 7$ \\
\hline Barlow (2005) & Case study & $\begin{array}{l}\text { Complexity } \\
\text { approach: } \\
\text { Complex clusters }\end{array}$ & $n=1(3 ; 9)$ & $\begin{array}{l}\text { Delivered by SLT student } \\
\text { at the participant's } \\
\text { home. }\end{array}$ & $\begin{array}{l}\text { 2-3 sessions per week, } \\
\text { each lasting } 45-60 \text { min. } \\
\text { Overall, } 19 \text { sessions } \\
\text { were delivered, each } \\
\text { comprising at least } 100 \\
\text { production trials. }\end{array}$ & $5 / 7$ \\
\hline $\begin{array}{l}\text { Bedore, Leonard and } \\
\text { Gandour (1994) }\end{array}$ & Case study & $\begin{array}{l}\text { Generic phonological } \\
\text { approach }^{\mathrm{d}}\end{array}$ & $n=1$ 'C' $(4 ; 4)$ & $\begin{array}{l}\text { Delivered in a university } \\
\text { clinic by an SLT. }\end{array}$ & $\begin{array}{l}2 \text { sessions per week, with a } \\
\text { total intervention } \\
\text { duration of } 4 \text { sessions } \\
\text { over } 2 \text { weeks. }\end{array}$ & $4 / 7$ \\
\hline $\begin{array}{l}\text { Bellon-Harn, Credeur- } \\
\text { Pampolina and } \\
\text { LeBoeuf (2013) }\end{array}$ & $\begin{array}{l}\text { Staggered MBD } \\
\text { across } \\
\text { participants }\end{array}$ & $\begin{array}{l}\text { Scaffolded language } \\
\text { intervention using } \\
\text { storybooks }\end{array}$ & $\begin{array}{c}n=2, \text { 'Casey' }(4 ; 8), \\
\text { 'Delbert' }(4 ; 2)\end{array}$ & $\begin{array}{l}\text { Delivered by SLT student } \\
\text { in a university clinic. }\end{array}$ & $\begin{array}{l}10 \times 20 \text {-min intervention } \\
\text { sessions. Dose reported } \\
\text { but unclear. }\end{array}$ & $4 / 7$ \\
\hline Bernhardt (1992) & $\begin{array}{l}\text { Combined MBD } \\
\text { across behaviours } \\
\text { with ATD }\end{array}$ & $\begin{array}{l}\text { Nonlinear } \\
\text { phonological } \\
\text { intervention }\end{array}$ & $n=1(5 ; 10)$ & $\begin{array}{l}\text { Individual intervention, } \\
\text { delivered by an SLT in } \\
\text { a university clinic. }\end{array}$ & $\begin{array}{l}3 \times 45 \text {-min sessions per } \\
\text { week for } 2 \times 6 \text { week } \\
\text { blocks. }\end{array}$ & $4 / 7$ \\
\hline
\end{tabular}




\begin{tabular}{|c|c|c|c|c|c|c|}
\hline Reference & Research design & $\begin{array}{l}\text { Intervention } \\
\text { approach }\end{array}$ & $\begin{array}{c}\text { Participant } \\
\text { number and age } \\
\text { (years;months) }\end{array}$ & $\begin{array}{c}\text { Summary of service } \\
\text { delivery }^{\mathrm{a}}\end{array}$ & $\begin{array}{l}\text { Summary of intervention } \\
\text { intensity }\end{array}$ & $\begin{array}{l}\text { Reporting of } \\
\text { intervention } \\
\text { intensity }^{b}\end{array}$ \\
\hline $\begin{array}{l}\text { Bernhardt and Major } \\
\text { (2005) }\end{array}$ & $\begin{array}{l}\text { Within subjects } \\
\text { design }\end{array}$ & $\begin{array}{l}\text { Nonlinear } \\
\text { phonological } \\
\text { intervention with } \\
\text { or without } \\
\text { metaphonological } \\
\text { intervention }\end{array}$ & $\begin{array}{l}n=12(3 ; 3-4 ; 11 \text { at } \\
\quad \text { time of } \\
\text { intervention, then } \\
6 ; 1-8 ; 5 \text { at time of } \\
\quad \text { follow-up) }\end{array}$ & $\begin{array}{l}\text { Individual intervention, } \\
\text { delivered by an SLT in } \\
\text { community clinics. }\end{array}$ & $\begin{array}{c}3 \times 45 \text {-min sessions per } \\
\text { week for } 16 \text { weeks. }\end{array}$ & $4 / 7$ \\
\hline $\begin{array}{l}\text { Blache, Parsons and } \\
\text { Humphreys (1981) }\end{array}$ & $\begin{array}{l}\text { Within subjects } \\
\text { design }\end{array}$ & Minimal pairs & $n=7(5 ; 4-6 ; 7)$ & $\begin{array}{l}\text { Individual intervention } \\
\text { delivered by SLT. }\end{array}$ & 3-5 sessions. & $2 / 7$ \\
\hline $\begin{array}{l}\text { Bowen and Cupples } \\
\quad \text { (1998) }\end{array}$ & Case study & $\begin{array}{l}\text { Parents and Children } \\
\text { Together (PACT) }\end{array}$ & $n=1$ 'Nina' $(4 ; 4)$ & $\begin{array}{l}\text { Individual intervention } \\
\text { delivered by an SLT in } \\
\text { a clinic. }\end{array}$ & $\begin{array}{l}27 \times 50 \text {-min consultations } \\
\text { (22 intervention and } 5 \\
\text { assessment) over } 17 \\
\text { months, delivered in } \\
\text { blocks of approx. } 8 \\
\text { sessions with approx. } \\
10 \text {-week breaks in } \\
\text { between the blocks. In } \\
\text { each block intervention } \\
\text { was delivered weekly. } \\
\text { Intervention was } \\
\text { provided from referral } \\
\text { to discharge. }\end{array}$ & $5 / 7$ \\
\hline $\begin{array}{l}\text { Bowen and Cupples } \\
\text { (1999a) }\end{array}$ & Non-RCT & $\begin{array}{c}\text { Parents and Children } \\
\text { Together (PACT) }\end{array}$ & $n=22(2 ; 11-4 ; 9)$ & $\begin{array}{l}\text { Individual intervention } \\
\text { delivered by an SLT in } \\
\text { a clinic. }\end{array}$ & $\begin{array}{l}1 \times 50 \text {-min session per } \\
\text { week, delivered in } \\
\text { blocks of } 10 \\
\text { consultations. Breaks of } \\
10 \text { weeks provided } \\
\text { between blocks of } \\
\text { intervention. Average of } \\
21 \text { consultations over } \\
10.6 \text { months. } \\
\text { Intervention was } \\
\text { provided from referral } \\
\text { to discharge. }\end{array}$ & $5 / 7$ \\
\hline
\end{tabular}




\begin{tabular}{|c|c|c|c|c|c|c|}
\hline Reference & Research design & $\begin{array}{l}\text { Intervention } \\
\text { approach }\end{array}$ & $\begin{array}{c}\text { Participant } \\
\text { number and age } \\
\text { (years;months) }\end{array}$ & $\begin{array}{c}\text { Summary of service } \\
\text { delivery }^{\mathbf{a}}\end{array}$ & $\begin{array}{l}\text { Summary of intervention } \\
\text { intensity }\end{array}$ & $\begin{array}{c}\text { Reporting of } \\
\text { intervention } \\
\text { intensity }^{\mathbf{b}}\end{array}$ \\
\hline $\begin{array}{l}\text { Bowen and Cupples } \\
\text { (1999b) }\end{array}$ & Case study & $\begin{array}{l}\text { Parents and Children } \\
\text { Together (PACT) }\end{array}$ & $n=1$ 'Cheri' $(4 ; 5)$ & $\begin{array}{l}\text { Individual intervention } \\
\text { delivered by an SLT in } \\
\text { a clinic. }\end{array}$ & $\begin{array}{l}1 \times 50 \text {-min session per } \\
\text { week. A total of } 23 \\
\text { sessions (18 } \\
\text { intervention and } 5 \\
\text { assessment) delivered } \\
\text { over } 5 \text { months. } \\
\text { Intervention was } \\
\text { provided from referral } \\
\text { to discharge. }\end{array}$ & $5 / 7$ \\
\hline $\begin{array}{l}\text { Broen and Westman } \\
\quad \text { (1990) }\end{array}$ & Non-RCT & $\begin{array}{l}\text { Generic phonological } \\
\text { approach }\end{array}$ & $\begin{array}{l}n=20 \\
\text { Experimental group } \\
\quad n=12(3 ; 7-5 ; 0) \\
\text { Control group } n=8 \\
\quad(3 ; 7-4 ; 8)\end{array}$ & $\begin{array}{l}\text { Group parent training } \\
\text { sessions delivered by } \\
\text { SLT, including some } \\
\text { individual intervention. } \\
\text { Most intervention } \\
\text { delivered at home by } \\
\text { parents. }\end{array}$ & $\begin{array}{l}1 \times 90 \text {-min group parent } \\
\text { training sessions per } \\
\text { week over approx. } 6 \\
\text { months }(17 \text { sessions in } \\
\text { total), each including } 5- \\
10 \text { mins of individual } \\
\text { intervention. }\end{array}$ & $5 / 7$ \\
\hline $\begin{array}{l}\text { Broomfield and Dodd } \\
\quad \text { (2011) }\end{array}$ & RCT & $\begin{array}{l}\text { Range of } \\
\text { intervention } \\
\text { approaches }\end{array}$ & $\begin{array}{l}n=730 \text { aged up to } \\
16 \text { years. Of } \\
\text { these, } n=320 \\
\text { received a } \\
\text { primary } \\
\text { diagnostic } \\
\text { category of } \\
\text { 'speech'. }\end{array}$ & $\begin{array}{l}\text { Intervention typically } \\
\text { delivered in groups by } \\
\text { two SLTs/SLT } \\
\text { assistants in a } \\
\text { community clinic. }\end{array}$ & $\begin{array}{l}\text { Average } 5.5 \text { hours of } \\
\text { intervention (range } 0- \\
24 \text { hours) over } 6 \\
\text { months. }\end{array}$ & $2 / 7$ \\
\hline $\begin{array}{l}\text { Bryan and Howard } \\
\text { (1992) }\end{array}$ & Case study & $\begin{array}{l}\text { Psycholinguistic } \\
\text { approach }\end{array}$ & $n=1$ 'DF' $(4 ; 10)$ & $\begin{array}{l}\text { Individual and group } \\
\text { intervention delivered } \\
\text { by an SLT in a } \\
\text { community clinic. }\end{array}$ & Delivered over 14 weeks. & $2 / 7$ \\
\hline Camarata (1993) & $\begin{array}{l}\text { MBD across } \\
\text { behaviours and } \\
\text { participants }\end{array}$ & $\begin{array}{l}\text { Naturalistic } \\
\text { intervention for } \\
\text { speech } \\
\text { intelligibility }\end{array}$ & $\begin{array}{c}n=2 \text { 'BH' }(3 ; 10), \\
\text { 'RM' }(4 ; 3)\end{array}$ & $\begin{array}{l}\text { Individual intervention } \\
\text { delivered by an SLT in } \\
\text { a university clinic. }\end{array}$ & $\begin{array}{c}2 \times 45 \text {-min sessions per } \\
\text { week. RM received } 14 \\
\text { sessions over } 7 \text { weeks } \\
\text { and BH received } 34 \\
\text { sessions over } 17 \text { weeks. }\end{array}$ & $5 / 7$ \\
\hline
\end{tabular}




\begin{tabular}{|c|c|c|c|c|c|c|}
\hline Reference & Research design & $\begin{array}{l}\text { Intervention } \\
\text { approach }\end{array}$ & $\begin{array}{c}\text { Participant } \\
\text { number and age } \\
\text { (years;months) }\end{array}$ & $\begin{array}{c}\text { Summary of service } \\
\text { delivery }^{\mathrm{a}}\end{array}$ & $\begin{array}{l}\text { Summary of intervention } \\
\text { intensity }^{\mathrm{a}}\end{array}$ & $\begin{array}{l}\text { Reporting of } \\
\text { intervention } \\
\text { intensity }^{b}\end{array}$ \\
\hline $\begin{array}{l}\text { Ceron, Keske-Soares, de } \\
\text { Freitas and Gubiani } \\
\text { (2010) }\end{array}$ & $\begin{array}{l}\text { Between groups } \\
\text { design }\end{array}$ & $\begin{array}{l}\text { ABAB withdrawal } \\
\text { and multiple } \\
\text { probes compared } \\
\text { with modified } \\
\text { maximal } \\
\text { oppositions } \\
\text { compared with } \\
\text { modified cycles }\end{array}$ & $n=21\left(\mathrm{M}_{\mathrm{age}}=5 ; 7\right)$ & $\begin{array}{l}\text { Delivered by an SLT in a } \\
\text { university clinic. }\end{array}$ & $\begin{array}{l}\text { 12-36 intervention } \\
\text { sessions. }\end{array}$ & $2 / 7$ \\
\hline $\begin{array}{l}\text { Ceron and Keske-Soares } \\
\text { (2013) }\end{array}$ & $\mathrm{AB}$ design & Multiple oppositions & $n=5(4 ; 2-8 ; 11)$ & $\begin{array}{l}\text { Delivered by an SLT in a } \\
\text { university clinic. }\end{array}$ & $\begin{array}{l}2 \times 45 \text {-min sessions per } \\
\text { week, for } 25 \text { sessions. } \\
\text { One participant was } \\
\text { discharged after } 15 \\
\text { sessions. Auditory } \\
\text { bombardment of } 20 \\
\text { words was provided at } \\
\text { the start and end of each } \\
\text { session (total } 40 \\
\text { perception dose). }\end{array}$ & $5 / 7$ \\
\hline $\begin{array}{l}\text { Checalin, Ghisleni, } \\
\text { Ferreira-Gonçalves, } \\
\text { Keske-Soares and } \\
\text { Mota (2010) }\end{array}$ & Case study & $\begin{array}{l}\text { ABAB withdrawal } \\
\text { and multiple } \\
\text { probes }\end{array}$ & $n=3(6 ; 0-7 ; 0)$ & $\begin{array}{l}\text { Delivered by an SLT in a } \\
\text { university clinic. }\end{array}$ & $\begin{array}{l}\text { Total intervention duration } \\
\text { of } 9 \text { sessions. }\end{array}$ & $2 / 7$ \\
\hline $\begin{array}{l}\text { Conture, Louko and } \\
\text { Edwards (1993) }\end{array}$ & Non-RCT & $\begin{array}{l}\text { Modified cycles with } \\
\text { minimal pairs and } \\
\text { articulation } \\
\text { intervention }\end{array}$ & $n=8\left(\mathrm{M}_{\mathrm{age}}=5 ; 9\right)$ & $\begin{array}{l}\text { Group intervention } \\
\text { delivered by two SLT } \\
\text { students in a university } \\
\text { clinic. Group parent } \\
\text { training was also } \\
\text { delivered. }\end{array}$ & $\begin{array}{l}1 \times 45 \text {-min session per } \\
\text { week during semester } \\
\text { time over a university } \\
\text { year. }\end{array}$ & $4 / 7$ \\
\hline $\begin{array}{l}\text { Crosbie , Holm and } \\
\text { Dodd (2005) }\end{array}$ & $\begin{array}{l}\text { Combined MBD } \\
\text { across behaviours } \\
\text { with ATD }\end{array}$ & $\begin{array}{l}\text { Minimal pairs } \\
\text { compared with } \\
\text { core vocabulary }\end{array}$ & $n=18(4 ; 8-6 ; 05)$ & $\begin{array}{l}\text { Individual intervention, } \\
\text { delivered by SLT at } \\
\text { school and home. }\end{array}$ & $\begin{array}{l}2 \times 30 \text {-min sessions per } \\
\text { week for } 8-9 \text { weeks per } \\
\text { intervention. A four } \\
\text { week break was } \\
\text { included between two } \\
\text { blocks of intervention. }\end{array}$ & $4 / 7$ \\
\hline
\end{tabular}




\begin{tabular}{|c|c|c|c|c|c|c|}
\hline Reference & Research design & $\begin{array}{l}\text { Intervention } \\
\text { approach }\end{array}$ & $\begin{array}{c}\text { Participant } \\
\text { number and age } \\
\text { (years;months) }\end{array}$ & $\begin{array}{c}\text { Summary of service } \\
\text { delivery }^{\mathrm{a}}\end{array}$ & $\begin{array}{c}\text { Summary of intervention } \\
\text { intensity }\end{array}$ & $\begin{array}{c}\text { Reporting of } \\
\text { intervention } \\
\text { intensity }\end{array}$ \\
\hline $\begin{array}{l}\text { Crosbie, Pine, Holm and } \\
\text { Dodd (2006) }\end{array}$ & Case study & Core vocabulary & $n=1$ 'Jarrod' $(7 ; 0)$ & $\begin{array}{l}\text { Individual intervention, } \\
\text { delivered by an SLT at } \\
\text { school. }\end{array}$ & $\begin{array}{l}2 \times 30 \text {-min sessions per } \\
\text { week for } 8 \text { weeks; } 16 \\
\text { sessions delivered in } \\
\text { total. Each session } \\
\text { comprised an average of } \\
100 \text { production trials. }\end{array}$ & $6 / 7$ \\
\hline $\begin{array}{l}\text { Culatta, Setzer and Horn } \\
\text { (2005) }\end{array}$ & Case study & Modified cycles & $n=1$ 'Casey' $(4 ; 2)$ & $\begin{array}{l}\text { Intervention delivered by } \\
\text { an SLT in a university } \\
\text { clinic }\end{array}$ & $\begin{array}{l}2 \times 50 \text {-min sessions per } \\
\text { week over } 9 \text { months. }\end{array}$ & $4 / 7$ \\
\hline $\begin{array}{l}\text { Cummings and Barlow } \\
\text { (2011) }\end{array}$ & $\begin{array}{l}\text { Staggered MBD } \\
\text { across } \\
\text { participants }\end{array}$ & $\begin{array}{l}\text { Complexity } \\
\text { approach: Non- } \\
\text { words }\end{array}$ & $n=4(3 ; 0-6 ; 9)$ & $\begin{array}{l}\text { Individual intervention, } \\
\text { delivered by an SLT in } \\
\text { a university clinic. }\end{array}$ & $\begin{array}{l}2 \times 60-\text { min sessions per } \\
\text { week, each comprising } \\
\text { a production dose of } \\
96-166 . \text { A total of } 19 \\
\text { sessions were provided } \\
\text { to each participant. }\end{array}$ & $5 / 7$ \\
\hline $\begin{array}{l}\text { Dean, Howell, Waters } \\
\text { and Reid (1995) }\end{array}$ & Case study & Metaphon & $n=13(3 ; 7-4 ; 7)$ & $\begin{array}{l}\text { Individual intervention, } \\
\text { delivered by an SLT in } \\
\text { a community clinic. }\end{array}$ & $\begin{array}{l}1 \times 30 \text {-min session per } \\
\text { week. An average of } 17 \\
\text { sessions were provided } \\
\text { over } 17 \text { weeks. }\end{array}$ & $5 / 7$ \\
\hline $\begin{array}{l}\text { Denne, Langdown, Pring } \\
\text { and Roy (2005) }\end{array}$ & RCT & $\begin{array}{l}\text { Phonological } \\
\text { awareness } \\
\text { intervention }\end{array}$ & $n=20(5-7)$ & $\begin{array}{l}\text { Group intervention, } \\
\text { delivered by an SLT in } \\
\text { a community clinic. }\end{array}$ & $\begin{array}{c}1 \times 90 \text {-min session per } \\
\text { week for } 8 \text { weeks. A } \\
\text { total of } 8 \text { sessions were } \\
\text { offered, resulting in } 12 \\
\text { hours of intervention. }\end{array}$ & $5 / 7$ \\
\hline $\begin{array}{l}\text { Derakhshandeh, } \\
\text { Nikmaram, } \\
\text { Hosseinabad, } \\
\text { Memarzadeh, Taheri, } \\
\text { Omrani, Jalaie, } \\
\text { Bijankhan and Sell }\end{array}$ & $\mathrm{ABA}$ & $\begin{array}{l}\text { Generic phonological } \\
\text { approach with } \\
\text { articulation } \\
\text { intervention }\end{array}$ & $n=5(4.5-9)$ & $\begin{array}{l}\text { Individual intervention, } \\
\text { delivered by an SLT in } \\
\text { a clinic. }\end{array}$ & $\begin{array}{l}4 \times 45 \text {-min sessions per } \\
\text { week for } 10 \text { weeks. } \\
\text { Total intervention } \\
\text { duration of } 40 \text { sessions } \\
\text { over } 10 \text { weeks. }\end{array}$ & $5 / 7$ \\
\hline
\end{tabular}




\begin{tabular}{|c|c|c|c|c|c|c|}
\hline Reference & Research design & $\begin{array}{l}\text { Intervention } \\
\text { approach }\end{array}$ & $\begin{array}{c}\text { Participant } \\
\text { number and age } \\
\text { (years;months) }\end{array}$ & $\begin{array}{c}\text { Summary of service } \\
\text { delivery }^{\mathrm{a}}\end{array}$ & $\begin{array}{l}\text { Summary of intervention } \\
\text { intensity }\end{array}$ & $\begin{array}{l}\text { Reporting of } \\
\text { intervention } \\
\text { intensity }^{b}\end{array}$ \\
\hline $\begin{array}{l}\text { Dinnsen, Chin and Elbert } \\
\text { (1992) }\end{array}$ & $\begin{array}{l}\text { MBD across } \\
\text { participants }\end{array}$ & Minimal pairs & $n=34(3 ; 4-6 ; 8)$ & $\begin{array}{l}\text { Individual intervention } \\
\text { delivered by an SLT. }\end{array}$ & $\begin{array}{l}2 \times 30 \text {-min session per } \\
\text { week; 83-409 days } \\
\text { (average 204) between } \\
\text { pre-intervention } \\
\text { assessment and post- } \\
\text { intervention assessment. }\end{array}$ & $4 / 7$ \\
\hline \multirow[t]{2}{*}{ Dodd and Barker (1990) } & $\begin{array}{l}\text { Within subjects } \\
\text { design }\end{array}$ & $\begin{array}{l}\text { Minimal pairs, } \\
\text { including } \\
\text { traditional } \\
\text { articulation }\end{array}$ & $\begin{array}{l}\text { Study } 1: n=5 \\
\quad(2 ; 1-4 ; 9)\end{array}$ & $\begin{array}{l}\text { Study 1: Parent-delivered } \\
\text { individual intervention } \\
\text { at home after receiving } \\
\text { training. }\end{array}$ & $\begin{array}{l}\text { Study } 1 \text { : } 1 \times 2 \text {-hour group } \\
\text { training session per } \\
\text { week for } 11 \text { weeks. }\end{array}$ & $5 / 7$ \\
\hline & & intervention & $\begin{array}{l}\text { Study } 2: n=6 \\
\quad(4 ; 0-4 ; 11)\end{array}$ & $\begin{array}{l}\text { Study 2: Teacher-delivered } \\
\text { individual intervention } \\
\text { at preschool after } \\
\text { receiving training. }\end{array}$ & $\begin{array}{l}\text { Study 2: Group teacher } \\
\text { training, consisting of } \\
\text { "an initial two-day } \\
\text { workshop, followed by } \\
\text { three half-day } \\
\text { workshops (each } \\
\text { between 2-4 hours } \\
\text { long) at approximately } \\
\text { three weekly intervals, } \\
\text { and a final half-day } \\
\text { workshop after a six } \\
\text { week break" (p. } 38 \text { ). }\end{array}$ & $5 / 7$ \\
\hline $\begin{array}{l}\text { Dodd and Bradford } \\
\text { (2000) }\end{array}$ & $\begin{array}{l}\text { Combined MBD } \\
\text { across behaviours } \\
\text { with ATD }\end{array}$ & $\begin{array}{l}\text { Metaphon compared } \\
\text { with PROMPT } \\
\text { compared with } \\
\text { core vocabulary }\end{array}$ & $n=3(3 ; 5-4 ; 3)$ & $\begin{array}{l}\text { Individual intervention } \\
\text { delivered by an SLT. }\end{array}$ & $\begin{array}{l}3 \times 6 \text {-week blocks of } \\
\text { intervention. Each block } \\
\text { consisted of } 12 \times 30- \\
\text { min sessions. }\end{array}$ & $4 / 7$ \\
\hline $\begin{array}{l}\text { Dodd, Crosbie, } \\
\text { McIntosh, Holm, } \\
\text { Harvey, Liddy, } \\
\text { Fontyne, Pinchin and }\end{array}$ & $\mathrm{RCT}$ & $\begin{array}{l}\text { Minimal pairs } \\
\text { compared with } \\
\text { non-minimal pairs }\end{array}$ & $n=19(3 ; 11-6 ; 5)$ & $\begin{array}{l}\text { Individual intervention } \\
\text { delivered by an SLT in } \\
\text { a community clinic. }\end{array}$ & $\begin{array}{l}1 \times 30 \text {-min session per } \\
\text { week for a total of } 12 \\
\text { sessions over } 12 \text { weeks. }\end{array}$ & $5 / 7$ \\
\hline
\end{tabular}




\begin{tabular}{|c|c|c|c|c|c|c|}
\hline Reference & Research design & $\begin{array}{l}\text { Intervention } \\
\text { approach }\end{array}$ & $\begin{array}{c}\text { Participant } \\
\text { number and age } \\
\text { (years;months) }\end{array}$ & $\begin{array}{c}\text { Summary of service } \\
\text { delivery }^{\text {a }}\end{array}$ & $\begin{array}{l}\text { Summary of intervention } \\
\text { intensity }\end{array}$ & $\begin{array}{l}\text { Reporting of } \\
\text { intervention } \\
\text { intensity }^{b}\end{array}$ \\
\hline Dodd and Iacano (1989) & Case study & Minimal pairs & $n=7(3 ; 0-4 ; 9)$ & $\begin{array}{l}\text { Individual intervention } \\
\text { delivered by an SLT in } \\
\text { a university clinic. For } \\
\text { one participant } \\
\text { ('Subject E') } \\
\text { intervention was } \\
\text { delivered at home by a } \\
\text { trained parent. }\end{array}$ & $\begin{array}{l}1 \times \text { session per week, for a } \\
\text { total of between } 11-40 \\
\text { sessions over } 3-16 \\
\text { months. }\end{array}$ & $4 / 7$ \\
\hline $\begin{array}{l}\text { Donicht, Pagliarin, Mota } \\
\text { and Keske-Soares } \\
\text { (2011) }\end{array}$ & Case study & $\begin{array}{l}\text { ABAB withdrawal } \\
\text { and multiple } \\
\text { probes compared } \\
\text { with complexity } \\
\text { approach: } \\
\text { Maximal } \\
\text { oppositions }\end{array}$ & $n=4(4 ; 0-6 ; 5)$ & $\begin{array}{l}\text { Individual intervention } \\
\text { delivered by an SLT in } \\
\text { a university clinic. }\end{array}$ & $\begin{array}{l}2 \times 45 \text {-min sessions per } \\
\text { week for } 5 \text { weeks, } \\
\text { totalling } 9 \text { intervention } \\
\text { sessions per cycle of } \\
\text { treatment. Participants } \\
\text { treated with the } \\
\text { maximal oppositions } \\
\text { approach received } 25 \\
\text { sessions of intervention. }\end{array}$ & $5 / 7$ \\
\hline Dunn and Barron (1982) & Case study & $\begin{array}{l}\text { Modified } \\
\text { McDonald's } \\
\text { (1964) approach }\end{array}$ & $n=1$ 'K' $(4 ; 11)$ & $\begin{array}{l}\text { Individual intervention } \\
\text { delivered by an SLT in } \\
\text { a university clinic. }\end{array}$ & $\begin{array}{l}2 \times 15 \text {-min sessions per } \\
\text { week for a total of } 16 \\
\text { sessions over } 8 \text { weeks. }\end{array}$ & $5 / 7$ \\
\hline $\begin{array}{l}\text { Eikeseth and Nesset } \\
\text { (2003) }\end{array}$ & Case study & $\begin{array}{l}\text { Vocal imitation } \\
\text { training }\end{array}$ & $n=2(5 ; 4-6 ; 0)$ & $\begin{array}{l}\text { Individual intervention } \\
\text { delivered by special } \\
\text { education students in } \\
\text { school or preschool. }\end{array}$ & $\begin{array}{l}\text { For participant } 1: 2 \times 2- \\
\text { hour sessions per week, } \\
\text { for a total of } 15 \text { sessions } \\
\text { over } 7.5 \text { weeks. } \\
\text { For participant } 2: 5 \times 2- \\
\text { hour sessions per week } \\
\text { for a total of } 21 \text { sessions } \\
\text { over } 29 \text { days. } \\
\text { For both participants, each } \\
\text { session contained } \\
\text { between } 80 \text { and } 200 \\
\text { production trials. }\end{array}$ & $6 / 7$ \\
\hline
\end{tabular}




\begin{tabular}{|c|c|c|c|c|c|c|}
\hline Reference & Research design & $\begin{array}{l}\text { Intervention } \\
\text { approach }\end{array}$ & $\begin{array}{c}\text { Participant } \\
\text { number and age } \\
\text { (years;months) }\end{array}$ & $\begin{array}{c}\text { Summary of service } \\
\text { delivery }^{\mathrm{a}}\end{array}$ & $\begin{array}{c}\text { Summary of intervention } \\
\text { intensity }\end{array}$ & $\begin{array}{l}\text { Reporting of } \\
\text { intervention } \\
\text { intensity }^{b}\end{array}$ \\
\hline $\begin{array}{l}\text { Eiserman, McCoun and } \\
\text { Escobar (1990) }\end{array}$ & RCT & $\begin{array}{l}\text { Generic phonological } \\
\text { approach }\end{array}$ & $n=40(3 ; 1-4 ; 10)$ & $\begin{array}{l}\text { For the clinic-based group, } \\
\text { intervention was } \\
\text { delivered by an SLT to } \\
\text { groups of } 2 \text { children in a } \\
\text { community clinic. } \\
\text { For the home parent } \\
\text { training group, } \\
\text { intervention was } \\
\text { primarily delivered by } \\
\text { parents. The SLT } \\
\text { provided training to } \\
\text { parents at home. }\end{array}$ & $\begin{array}{l}\text { For the clinic-based group, } \\
1 \times 1 \text {-hour session per } \\
\text { week for } 7 \text { months. } \\
\text { For the home parent } \\
\text { training group, } 2 \times 40- \\
\text { min training sessions } \\
\text { per month for } 7 \text { months. }\end{array}$ & $4 / 7$ \\
\hline $\begin{array}{l}\text { Eiserman, Weber and } \\
\text { McCoun (1992) }\end{array}$ & $\begin{array}{l}\text { RCT (follow-up to } \\
\text { Eiserman et al., } \\
\text { 1990) }\end{array}$ & $\begin{array}{l}\text { Generic phonological } \\
\text { approach }\end{array}$ & $\begin{array}{l}n=40(3 ; 1-4 ; 10 \text { at } \\
\text { time of } \\
\text { intervention, then } \\
\text { followed up one } \\
\text { year later) }\end{array}$ & $\begin{array}{l}\text { As per Eiserman et al. } \\
\quad(1990) \text {. }\end{array}$ & $\begin{array}{l}\text { As per Eiserman et al. } \\
\text { (1990). }\end{array}$ & $4 / 7$ \\
\hline Elbert (1983) & Case study & $\begin{array}{l}\text { Generic phonological } \\
\text { approach }\end{array}$ & $\begin{array}{c}n=1 \text { 'Matthew' } \\
(3 ; 10)\end{array}$ & $\begin{array}{l}\text { Individual intervention } \\
\text { delivered by an SLT in } \\
\text { a university clinic. }\end{array}$ & $\begin{array}{l}\text { 2-3 sessions per week over } \\
11 \text { months, with } \\
\text { periodic breaks from } \\
\text { intervention. }\end{array}$ & 237 \\
\hline $\begin{array}{l}\text { Elbert, Dinnsen, } \\
\text { Swartzlander and } \\
\text { Chin (1990) }\end{array}$ & $\begin{array}{l}\text { Within subjects } \\
\text { design }\end{array}$ & Minimal pairs & $n=10(3 ; 7-5 ; 9)$ & $\begin{array}{l}\text { Individual intervention } \\
\text { delivered by an SLT in } \\
\text { a university clinic. }\end{array}$ & $\begin{array}{l}2 \times 30 \text {-min sessions per } \\
\text { week. }\end{array}$ & $3 / 7$ \\
\hline $\begin{array}{l}\text { Elbert, Powell and } \\
\text { Swartzlander (1991) }\end{array}$ & $\begin{array}{l}\text { Within subjects } \\
\text { design }\end{array}$ & Minimal pairs & $n=19(4 ; 0-6 ; 7)$ & $\begin{array}{l}\text { Individual intervention, } \\
\text { delivered by an SLT in } \\
\text { a university clinic. }\end{array}$ & $\begin{array}{l}2 \times 30 \text {-min sessions per } \\
\text { week. Approx. } 80-100 \\
\text { production trials were } \\
\text { elicited in each session. } \\
\text { Cumulative intervention } \\
\text { intensity of } 180-2840 \\
\text { production trials. }\end{array}$ & $5 / 7$ \\
\hline
\end{tabular}




\begin{tabular}{|c|c|c|c|c|c|c|}
\hline Reference & Research design & $\begin{array}{l}\text { Intervention } \\
\text { approach }\end{array}$ & $\begin{array}{c}\text { Participant } \\
\text { number and age } \\
\text { (years;months) }\end{array}$ & $\begin{array}{c}\text { Summary of service } \\
\text { delivery }^{\mathrm{a}}\end{array}$ & $\begin{array}{l}\text { Summary of intervention } \\
\text { intensity }\end{array}$ & $\begin{array}{l}\text { Reporting of } \\
\text { intervention } \\
\text { intensity }^{b}\end{array}$ \\
\hline $\begin{array}{l}\text { Feehan, Francis, } \\
\text { Bernhardt and } \\
\text { Colozzo }(2015)\end{array}$ & Case study & $\begin{array}{l}\text { Modified cycles and } \\
\text { morphosyntax } \\
\text { intervention }\end{array}$ & $n=2($ twins, $6 ; 7)$ & $\begin{array}{l}\text { Individual intervention, } \\
\text { delivered by an SLT } \\
\text { student, in a university } \\
\text { clinic. }\end{array}$ & $\begin{array}{l}\text { Each child received } 2 \\
\text { blocks of intervention. } \\
\text { Each block comprised } 1 \\
\times 60 \text {-min session per } \\
\text { week for } 8 \text { weeks. } \\
\text { Children received a total } \\
\text { of } 16 \text { sessions over } 23 \\
\text { weeks. }\end{array}$ & $5 / 7$ \\
\hline $\begin{array}{l}\text { Fey, Cleave, Ravida, } \\
\text { Long, Dejmal and } \\
\text { Easton (1994) }\end{array}$ & RCT & $\begin{array}{l}\text { Grammar } \\
\text { intervention using } \\
\text { focused } \\
\text { stimulation } \\
\text { techniques }\end{array}$ & $n=26(3 ; 8-5 ; 10)$ & $\begin{array}{l}\text { Treatment group 1: } \\
\text { Individual and group } \\
\text { sessions, delivered by } \\
\text { an SLT. } \\
\text { Treatment group 2: Parent- } \\
\text { only group training } \\
\text { sessions delivered by an } \\
\text { SLT, and individual } \\
\text { intervention delivered } \\
\text { by an SLT. } \\
\text { Treatment group 3: } \\
\text { delayed treatment } \\
\text { group. Randomly } \\
\text { allocated to the same } \\
\text { treatment as group } 1 \text { or } \\
\text { 2, above. }\end{array}$ & $\begin{array}{l}\text { Treatment group } 1 \text { : } 3 \times 1- \\
\text { hour sessions per week } \\
\text { over a total intervention } \\
\text { duration of } 10 \text { months. } \\
\text { Treatment group } 2: 1 \times 2- \\
\text { hour parent training } \\
\text { sessions per week for } 12 \\
\text { weeks. Following this } \\
12 \text {-week period, parents } \\
\text { attended } 1 \times \text { group } \\
\text { parent training session } \\
\text { per month and children } \\
\text { received } 1 \times \text { individual } \\
\text { session per month. Total } \\
\text { intervention duration of } \\
10 \text { months. } \\
\text { Treatment group } 3 \text { : } \\
\text { delayed treatment } \\
\text { group. Total } \\
\text { intervention duration of } \\
5 \text { months. }\end{array}$ & $4 / 7$ \\
\hline Fey and Stalker (1986) & Case study & Minimal pairs & $n=1$ 'Nora' $(6 ; 9)$ & $\begin{array}{l}\text { Individual intervention } \\
\text { delivered by an SLT in } \\
\text { a university clinic. }\end{array}$ & $\begin{array}{l}2 \times 90 \text {-min sessions per } \\
\text { week. Breaks in } \\
\text { intervention due to } \\
\text { holiday periods. Total } \\
\text { intervention duration of } \\
46 \text { hours over } 8.5 \\
\text { months. }\end{array}$ & $4 / 7$ \\
\hline
\end{tabular}




\begin{tabular}{|c|c|c|c|c|c|c|}
\hline Reference & Research design & $\begin{array}{l}\text { Intervention } \\
\text { approach }\end{array}$ & $\begin{array}{c}\text { Participant } \\
\text { number and age } \\
\text { (years;months) }\end{array}$ & $\begin{array}{c}\text { Summary of service } \\
\text { delivery }^{\mathrm{a}}\end{array}$ & $\begin{array}{l}\text { Summary of intervention } \\
\text { intensity }\end{array}$ & $\begin{array}{l}\text { Reporting of } \\
\text { intervention } \\
\text { intensity }^{b}\end{array}$ \\
\hline $\begin{array}{l}\text { Flint and Costello } \\
\text { Ingham (2005) }\end{array}$ & $\begin{array}{l}\text { Within subjects } \\
\text { design }\end{array}$ & $\begin{array}{l}\text { Generic phonological } \\
\text { approach }\end{array}$ & $n=7(4 ; 0-5 ; 7)$ & $\begin{array}{l}\text { Individual intervention } \\
\text { delivered by an SLT in } \\
\text { a university clinic. }\end{array}$ & $\begin{array}{l}3 \times 30-50 \text {-min sessions } \\
\text { per week. }\end{array}$ & $3 / 7$ \\
\hline $\begin{array}{l}\text { Forrest, Dinnsen and } \\
\quad \text { Elbert (1997) }\end{array}$ & $\begin{array}{l}\text { Within subjects } \\
\text { design }\end{array}$ & Minimal pairs & $n=14(3 ; 6-5 ; 9)$ & $\begin{array}{l}\text { Individual intervention } \\
\text { delivered by an SLT in } \\
\text { a university clinic. }\end{array}$ & $\begin{array}{l}2 \times 30 \text {-min sessions per } \\
\text { week, up to a maximum } \\
\text { of } 20 \text { sessions. }\end{array}$ & $4 / 7$ \\
\hline Forrest and Elbert (2001) & $\begin{array}{l}\text { MBD across } \\
\text { behaviours }\end{array}$ & $\begin{array}{l}\text { Generic phonological } \\
\text { approach }\end{array}$ & $n=4(4 ; 11-5 ; 3)$ & $\begin{array}{l}\text { Individual intervention } \\
\text { delivered by an SLT in } \\
\text { a university clinic. }\end{array}$ & $\begin{array}{l}2 \times 45 \text {-min sessions per } \\
\text { week. }\end{array}$ & $3 / 7$ \\
\hline $\begin{array}{l}\text { Forrest, Elbert and } \\
\text { Dinnsen (2000) }\end{array}$ & $\begin{array}{l}\text { Staggered MBD } \\
\text { across } \\
\text { participants }\end{array}$ & $\begin{array}{l}\text { Generic phonological } \\
\text { approach }\end{array}$ & $n=10(3 ; 4-4 ; 6)$ & $\begin{array}{l}\text { Individual intervention } \\
\text { delivered by a student } \\
\text { SLT in a university } \\
\text { clinic. }\end{array}$ & $\begin{array}{l}2 \times \text { sessions per week, for } \\
\text { between } 9 \text { and } 20 \\
\text { sessions. }\end{array}$ & $3 / 7$ \\
\hline $\begin{array}{l}\text { Gibbon, Shockey and } \\
\text { Reid (1992) }\end{array}$ & Case study & Vowel intervention & $n=1$ 'Danny' $(4 ; 0)$ & $\begin{array}{l}\text { Individual intervention } \\
\text { delivered by an SLT in } \\
\text { a university clinic. }\end{array}$ & $\begin{array}{c}1 \times 45 \text {-min session per } \\
\text { week for a total of } 6 \\
\text { sessions over } 6 \text { weeks. }\end{array}$ & $5 / 7$ \\
\hline Gierut (1989) & $\begin{array}{l}\text { MBD across } \\
\text { behaviours }\end{array}$ & $\begin{array}{l}\text { Complexity } \\
\text { approach: } \\
\text { Maximal } \\
\text { oppositions }\end{array}$ & $n=1$ 'J' $(4 ; 7)$ & $\begin{array}{l}\text { Individual intervention } \\
\text { delivered by an SLT in } \\
\text { a university clinic. }\end{array}$ & $\begin{array}{l}2 \times 30 \text {-min sessions per } \\
\text { week, for } 23 \text { sessions } \\
\text { over a } 3 \text {-month period. }\end{array}$ & $5 / 7$ \\
\hline Gierut (1990) & $\begin{array}{l}\text { Combined ATD } \\
\text { with staggered } \\
\text { MBD across } \\
\text { participants }\end{array}$ & $\begin{array}{l}\text { Complexity } \\
\text { approach: } \\
\text { Maximal } \\
\text { oppositions } \\
\text { compared with } \\
\text { minimal pairs }\end{array}$ & $n=3(4 ; 1-4 ; 10)$ & $\begin{array}{l}\text { Individual intervention } \\
\text { delivered by an SLT in } \\
\text { a university clinic. }\end{array}$ & $\begin{array}{l}3 \times 60 \text {-min sessions per } \\
\text { week. }\end{array}$ & $3 / 7$ \\
\hline Gierut (1991) & $\begin{array}{l}\text { Combined ATD } \\
\text { with staggered } \\
\text { MBD across } \\
\text { participants }\end{array}$ & $\begin{array}{l}\text { Complexity } \\
\text { approach: Empty } \\
\text { set compared with } \\
\text { minimal pairs }\end{array}$ & $n=3(4 ; 2-5 ; 4)$ & $\begin{array}{l}\text { Individual intervention } \\
\text { delivered by an SLT in } \\
\text { a university clinic. }\end{array}$ & $\begin{array}{l}3 \times 60 \text {-min sessions per } \\
\text { week for a total of } 17- \\
19 \text { sessions. }\end{array}$ & $4 / 7$ \\
\hline Gierut (1992) & $\begin{array}{l}\text { Combined ATD } \\
\text { with staggered } \\
\text { MBD across } \\
\text { participants }\end{array}$ & $\begin{array}{l}\text { Complexity } \\
\text { approach: Empty } \\
\text { set compared with } \\
\text { maximal } \\
\text { oppositions }\end{array}$ & $n=4(3 ; 6-4 ; 2)$ & $\begin{array}{l}\text { Individual intervention } \\
\text { delivered by an SLT in } \\
\text { a university clinic. }\end{array}$ & $\begin{array}{l}3 \times 60 \text {-min sessions per } \\
\text { week for a total of } 19 \\
\text { sessions over a } \\
\text { maximum of } 7 \text { weeks. }\end{array}$ & $5 / 7$ \\
\hline
\end{tabular}




\begin{tabular}{|c|c|c|c|c|c|c|}
\hline Reference & Research design & $\begin{array}{l}\text { Intervention } \\
\text { approach }\end{array}$ & $\begin{array}{c}\text { Participant } \\
\text { number and age } \\
\text { (years;months) }\end{array}$ & $\begin{array}{c}\text { Summary of service } \\
\text { delivery }^{\mathrm{a}}\end{array}$ & $\begin{array}{l}\text { Summary of intervention } \\
\text { intensity }\end{array}$ & $\begin{array}{c}\text { Reporting of } \\
\text { intervention } \\
\text { intensity }^{\mathbf{b}}\end{array}$ \\
\hline Gierut (1996) & $\begin{array}{l}\text { Staggered MBD } \\
\text { across } \\
\text { participants }\end{array}$ & $\begin{array}{l}\text { Complexity } \\
\text { approach: } \\
\text { Laryngeal/ } \\
\text { supralaryngeal } \\
\text { distinctions }\end{array}$ & $n=7(3 ; 4-5 ; 8)$ & $\begin{array}{l}\text { Individual intervention } \\
\text { delivered by an SLT in } \\
\text { a university clinic. }\end{array}$ & $\begin{array}{l}3 \times 60 \text {-min sessions per } \\
\text { week over } 4.5 \text { months. }\end{array}$ & $4 / 7$ \\
\hline Gierut (1998) & $\begin{array}{l}\text { Combined staggered } \\
\text { MBD with MPD }\end{array}$ & $\begin{array}{l}\text { Complexity } \\
\text { approach: } \\
\text { singletons and/or } \\
\text { clusters involving } \\
\text { laryngeal / } \\
\text { supralaryngeal } \\
\text { distinctions }\end{array}$ & $n=6(3 ; 2-7 ; 3)$ & $\begin{array}{l}\text { Individual intervention } \\
\text { delivered by an SLT in } \\
\text { a university clinic. }\end{array}$ & $\begin{array}{l}3 \times 60 \text {-min sessions per } \\
\text { week for an average of } \\
16 \text { sessions delivered } \\
\text { over a maximum of } 6 \\
\text { weeks. Each session } \\
\text { comprised } 100 \\
\text { production trials. }\end{array}$ & $6 / 7$ \\
\hline \multirow[t]{2}{*}{ Gierut (1999) } & $\begin{array}{l}\text { Staggered MBD } \\
\text { across } \\
\text { participants }\end{array}$ & $\begin{array}{l}\text { Complexity } \\
\text { approach: } \\
\text { Sonority } \\
\text { differences within } \\
\text { clusters }\end{array}$ & $\begin{array}{l}\text { Experiment } 1: n=6 \\
\quad(3 ; 2-7 ; 8)\end{array}$ & $\begin{array}{l}\text { Experiment 1: Individual } \\
\text { intervention delivered } \\
\text { by an SLT in a } \\
\text { university clinic. }\end{array}$ & $\begin{array}{l}\text { Experiment } 1: 3 \times 60 \text {-min } \\
\text { sessions per week for up } \\
\text { to } 19 \text { sessions over a } \\
\text { maximum of } 7 \text { weeks. }\end{array}$ & $5 / 7$ \\
\hline & & & $\begin{array}{l}\text { Experiment } 2: n=5 \\
\quad(3 ; 5-4 ; 8)\end{array}$ & $\begin{array}{l}\text { Experiment 2: Individual } \\
\text { intervention delivered } \\
\text { by an SLT in a } \\
\text { university clinic. }\end{array}$ & $\begin{array}{l}\text { Experiment } 2: 3 \times 60 \text {-min } \\
\text { sessions per week for up } \\
\text { to } 19 \text { sessions over a } \\
\text { maximum of } 7 \text { weeks. }\end{array}$ & $5 / 7$ \\
\hline $\begin{array}{l}\text { Gierut and Champion } \\
\text { (1999) }\end{array}$ & $\begin{array}{l}\text { Staggered MBD } \\
\text { across } \\
\text { participants }\end{array}$ & $\begin{array}{l}\text { Complexity } \\
\text { approach: Chain } \\
\text { shifts }\end{array}$ & $n=2(4 ; 0-4 ; 8)$ & $\begin{array}{l}\text { Individual intervention } \\
\text { delivered by an SLT in } \\
\text { a university clinic. }\end{array}$ & $\begin{array}{l}3 \times 60 \text {-min sessions per } \\
\text { week for a total of } 19 \\
\text { sessions delivered over } \\
\text { approx. } 7 \text { weeks. }\end{array}$ & $5 / 7$ \\
\hline $\begin{array}{l}\text { Gierut and Champion } \\
\text { (2000) }\end{array}$ & $\begin{array}{l}\mathrm{AB} \text { design with } \\
\text { control behaviour }\end{array}$ & $\begin{array}{l}\text { Generic phonological } \\
\text { approach }\end{array}$ & $n=1$ 'IJ' $(4 ; 5)$ & $\begin{array}{l}\text { Individual intervention } \\
\text { delivered by an SLT in } \\
\text { a university clinic. }\end{array}$ & $\begin{array}{l}3 \times 60 \text {-min sessions per } \\
\text { week for a total of } 19 \\
\text { sessions delivered over } \\
\text { approx. } 7 \text { weeks. }\end{array}$ & $5 / 7$ \\
\hline $\begin{array}{l}\text { Gierut and Champion } \\
\quad(2001)\end{array}$ & $\begin{array}{l}\text { Staggered MBD } \\
\text { across } \\
\text { participants }\end{array}$ & $\begin{array}{l}\text { Complexity } \\
\text { approach: } 3 \text { - } \\
\text { element clusters }\end{array}$ & $n=8(3 ; 4-6 ; 3)$ & $\begin{array}{l}\text { Individual intervention } \\
\text { delivered by an SLT in } \\
\text { a university clinic. }\end{array}$ & $\begin{array}{l}3 \times 60 \text {-min sessions per } \\
\text { week for a total of } 16- \\
19 \text { sessions over a } \\
\text { maximum of } 7 \text { weeks. }\end{array}$ & $5 / 7$ \\
\hline
\end{tabular}




\begin{tabular}{|c|c|c|c|c|c|c|}
\hline Reference & Research design & $\begin{array}{l}\text { Intervention } \\
\text { approach }\end{array}$ & $\begin{array}{c}\text { Participant } \\
\text { number and age } \\
\text { (years;months) }\end{array}$ & $\begin{array}{c}\text { Summary of service } \\
\text { delivery }^{\mathrm{a}}\end{array}$ & $\begin{array}{l}\text { Summary of intervention } \\
\text { intensity }^{\mathrm{a}}\end{array}$ & $\begin{array}{l}\text { Reporting of } \\
\text { intervention } \\
\text { intensity }^{b}\end{array}$ \\
\hline $\begin{array}{l}\text { Gierut, Elbert and } \\
\text { Dinnsen (1987) }\end{array}$ & $\begin{array}{l}\text { Combined MBD } \\
\text { across } \\
\text { participants with } \\
\text { MPD }\end{array}$ & Minimal pairs & $n=6(3 ; 7-4 ; 6)$ & $\begin{array}{l}\text { Individual intervention } \\
\text { delivered by an SLT in } \\
\text { a university clinic. }\end{array}$ & $\begin{array}{l}\text { 30-min sessions, each } \\
\text { comprising approx. } 150 \\
\text { production trials. }\end{array}$ & $3 / 7$ \\
\hline $\begin{array}{l}\text { Gierut and Morrisette } \\
\text { (1996) }\end{array}$ & $\begin{array}{l}\text { Combined staggered } \\
\text { MBD across } \\
\text { participants with } \\
\text { MPD }\end{array}$ & $\begin{array}{l}\text { Complexity } \\
\text { approach: } \\
\text { Laryngeal/ } \\
\text { supralaryngeal } \\
\text { distinctions }\end{array}$ & $n=2(4 ; 5-5 ; 8)$ & $\begin{array}{l}\text { Individual intervention } \\
\text { delivered by an SLT in } \\
\text { a university clinic. }\end{array}$ & $\begin{array}{c}3 \times 60-\text { min sessions per } \\
\text { week, each comprising } \\
100 \text { production trials. }\end{array}$ & $4 / 7$ \\
\hline $\begin{array}{l}\text { Gierut and Morrisette } \\
\quad(2010)\end{array}$ & $\begin{array}{l}\text { Combined ATD } \\
\text { with staggered } \\
\text { MBD across } \\
\text { participants }\end{array}$ & $\begin{array}{l}\text { Complexity } \\
\text { approach: non- } \\
\text { words }\end{array}$ & $n=4(3 ; 6-5 ; 7)$ & $\begin{array}{l}\text { Individual intervention } \\
\text { delivered by an SLT in } \\
\text { a university clinic. }\end{array}$ & $\begin{array}{l}3 \times 60 \text {-min sessions per } \\
\text { week for a total of } 5-19 \\
\text { sessions delivered over } \\
\text { approx. } 7 \text { weeks. Each } \\
\text { session comprised an } \\
\text { average of } 62 \\
\text { production trials. }\end{array}$ & $6 / 7$ \\
\hline $\begin{array}{l}\text { Gierut and Morrisette } \\
\quad(2012 a)\end{array}$ & $\begin{array}{l}\text { Staggered MBD } \\
\text { across } \\
\text { participants }\end{array}$ & $\begin{array}{l}\text { Complexity } \\
\text { approach: Age of } \\
\text { acquisition }\end{array}$ & $n=10(3 ; 10-5 ; 11)$ & $\begin{array}{l}\text { Individual intervention } \\
\text { delivered by an SLT in } \\
\text { a university clinic. }\end{array}$ & $\begin{array}{l}3 \text { sessions per week, for a } \\
\text { maximum of } 19 \\
\text { sessions. }\end{array}$ & $3 / 7$ \\
\hline $\begin{array}{l}\text { Gierut and Morrisette } \\
\qquad(2012 b)\end{array}$ & $\begin{array}{l}\text { Staggered MBD } \\
\text { across } \\
\text { participants }\end{array}$ & $\begin{array}{l}\text { Complexity } \\
\text { approach: Density } \\
\text { and frequency }\end{array}$ & $n=8(3 ; 0-5 ; 5)$ & $\begin{array}{l}\text { Individual intervention } \\
\text { delivered by an SLT in } \\
\text { a university clinic. }\end{array}$ & $\begin{array}{l}3 \times 60 \text {-min sessions per } \\
\text { week, for a total of } 7- \\
19 \text { sessions (average } 15 \text { ) } \\
\text { delivered over a } \\
\text { maximum of } 7 \text { weeks. }\end{array}$ & $5 / 7$ \\
\hline $\begin{array}{l}\text { Gierut and Morrisette } \\
\text { (2014) }\end{array}$ & $\begin{array}{l}\text { Staggered MBD } \\
\text { across } \\
\text { participants }\end{array}$ & $\begin{array}{l}\text { Complexity } \\
\text { approach: Dense } \\
\text { neighbours with } \\
\text { priming }\end{array}$ & $n=9(3 ; 5-5 ; 7)$ & $\begin{array}{l}\text { Individual intervention } \\
\text { delivered by an SLT in } \\
\text { a university clinic. }\end{array}$ & $\begin{array}{l}3 \times 60 \text {-min sessions per } \\
\text { week for a total of } 5- \\
19 \text { sessions delivered } \\
\text { over approx. } 2-7 \\
\text { weeks. }\end{array}$ & $5 / 7$ \\
\hline
\end{tabular}




\begin{tabular}{|c|c|c|c|c|c|c|}
\hline Reference & Research design & $\begin{array}{l}\text { Intervention } \\
\text { approach }\end{array}$ & $\begin{array}{c}\text { Participant } \\
\text { number and age } \\
\text { (years;months) }\end{array}$ & $\begin{array}{c}\text { Summary of service } \\
\text { delivery }^{\mathrm{a}}\end{array}$ & $\begin{array}{l}\text { Summary of intervention } \\
\text { intensity }^{\mathrm{a}}\end{array}$ & $\begin{array}{c}\text { Reporting of } \\
\text { intervention } \\
\text { intensity }^{\mathbf{b}}\end{array}$ \\
\hline $\begin{array}{l}\text { Gierut and Morrisette } \\
\text { (2015) }\end{array}$ & $\begin{array}{l}\text { Staggered MBD } \\
\text { across } \\
\text { participants }\end{array}$ & $\begin{array}{l}\text { Complexity } \\
\text { approach: Dense } \\
\text { neighbours with } \\
\text { priming }\end{array}$ & $\begin{array}{l}\text { Study } 1: n=6 \\
\quad(3 ; 5-4 ; 7)\end{array}$ & $\begin{array}{l}\text { Study 1: Individual } \\
\text { intervention delivered } \\
\text { by an SLT in a } \\
\text { university clinic. }\end{array}$ & $\begin{array}{l}\text { Study 1: } 3 \times 60 \text {-min } \\
\text { sessions per week for a } \\
\text { total of 6-19 sessions } \\
\text { (average 15) delivered } \\
\text { over approx. } 2-7 \text { weeks. } \\
\text { Each session comprised } \\
\text { an average of } 71 \\
\text { production trials. }\end{array}$ & $6 / 7$ \\
\hline $\begin{array}{l}\text { Gierut, Morrisette and } \\
\text { Champion (1999) }\end{array}$ & $\begin{array}{l}\text { Combined ATD } \\
\text { with MBD across } \\
\text { participants }\end{array}$ & $\begin{array}{l}\text { Complexity } \\
\text { approach: } \\
\text { Frequency and } \\
\text { neighbourhood } \\
\text { density }\end{array}$ & $n=12(3 ; 0-7 ; 4)$ & $\begin{array}{l}\text { Individual intervention } \\
\text { delivered by an SLT in } \\
\text { a university clinic. }\end{array}$ & $\begin{array}{l}3 \times 60 \text {-min sessions per } \\
\text { week for up to } 19 \\
\text { sessions. }\end{array}$ & $4 / 7$ \\
\hline & $\begin{array}{l}\text { participants } \\
\text { Study 2: Staggered } \\
\text { MBD across } \\
\text { participants }\end{array}$ & $\begin{array}{l}\text { acquired } \\
\text { phonemes }\end{array}$ & $\begin{array}{l}\text { Study } 2: n=6 \\
\quad(3 ; 5-5 ; 6)\end{array}$ & $\begin{array}{l}\text { university clinic. } \\
\text { Study 2: Individual } \\
\text { intervention delivered } \\
\text { by an SLT in a } \\
\text { university clinic. }\end{array}$ & $\begin{array}{l}\text { Study } 2: 3 \times 60 \text {-min } \\
\text { sessions per week for up } \\
\text { to } 19 \text { sessions. }\end{array}$ & $4 / 7$ \\
\hline $\begin{array}{l}\text { Gierut, Morrisette and } \\
\text { Ziemer (2010) }\end{array}$ & $\begin{array}{l}\text { Retrospective } \\
\text { analysis (between } \\
\text { groups) }\end{array}$ & $\begin{array}{l}\text { Complexity } \\
\text { approach: Real- } \\
\text { words versus non- } \\
\text { words }\end{array}$ & $n=60(3 ; 1-7 ; 5)$ & $\begin{array}{l}\text { Individual intervention } \\
\text { delivered by an SLT in } \\
\text { a university clinic. }\end{array}$ & $\begin{array}{l}3 \times 1 \text {-hour sessions per } \\
\text { week, for an average of } \\
13 \text { (treatment using real- } \\
\text { words group) or } 15 \\
\text { sessions (non-words } \\
\text { group) for up to a total } \\
\text { of } 19 \text { sessions. }\end{array}$ & $4 / 7$ \\
\hline
\end{tabular}




\begin{tabular}{|c|c|c|c|c|c|c|}
\hline Reference & Research design & $\begin{array}{l}\text { Intervention } \\
\text { approach }\end{array}$ & $\begin{array}{c}\text { Participant } \\
\text { number and age } \\
\text { (years;months) }\end{array}$ & $\begin{array}{c}\text { Summary of service } \\
\text { delivery }^{\mathrm{a}}\end{array}$ & $\begin{array}{l}\text { Summary of intervention } \\
\text { intensity }\end{array}$ & $\begin{array}{l}\text { Reporting of } \\
\text { intervention } \\
\text { intensity }^{b}\end{array}$ \\
\hline $\begin{array}{l}\text { Gierut and Neumann } \\
\text { (1992) }\end{array}$ & ATD & $\begin{array}{l}\text { Complexity } \\
\text { approach: Empty } \\
\text { set compared with } \\
\text { minimal pairs }\end{array}$ & $n=1(4 ; 8)$ & $\begin{array}{l}\text { Individual intervention } \\
\text { delivered by an SLT in } \\
\text { a university clinic. }\end{array}$ & $\begin{array}{l}3 \times 1 \text {-hour sessions per } \\
\text { week for a total of } 13 \\
\text { sessions. }\end{array}$ & $4 / 7$ \\
\hline $\begin{array}{l}\text { Gildersleeve-Neumann } \\
\text { and Goldstein (2015) }\end{array}$ & $\begin{array}{l}\text { Multiple probe } \\
\text { across behaviours }\end{array}$ & $\begin{array}{l}\text { Generic phonological } \\
\text { approach } \\
\text { combined with } \\
\text { elements of } \\
\text { articulation } \\
\text { therapy }\end{array}$ & $n=2(5 ; 6-5 ; 8)$ & $\begin{array}{l}\text { Individual intervention } \\
\text { delivered by an SLT } \\
\text { student in a university } \\
\text { clinic. }\end{array}$ & $\begin{array}{l}2-3 \times 50 \text {-min sessions per } \\
\text { week for } 8 \text { weeks. } \\
\text { Intervention delivered } \\
\text { for a total of } 19 \text { or } 25 \\
\text { sessions, each } \\
\text { comprising } 40-100 \\
\text { production trials. } \\
\text { Cumulative intervention } \\
\text { intensity inferred to be } \\
\text { approximately } 1330 \\
\text { production trials for one } \\
\text { participant and } 1750 \text { for } \\
\text { the second participant. }\end{array}$ & $7 / 7$ \\
\hline Gillon (2000) & Non-RCT & $\begin{array}{l}\text { Phonological } \\
\text { awareness } \\
\text { intervention }\end{array}$ & $n=91(5 ; 6-7 ; 6)$ & $\begin{array}{l}\text { Experimental group: } \\
\text { Individual intervention } \\
\text { delivered by an SLT in } \\
\text { a university clinic or a } \\
\text { local community clinic. }\end{array}$ & $\begin{array}{l}2 \times 60 \text {-min sessions per } \\
\text { week for a total of } 20 \\
\text { sessions over an average } \\
\text { of } 4.5 \text { months. }\end{array}$ & $5 / 7$ \\
\hline Gillon (2005) & Non-RCT & $\begin{array}{l}\text { Cycles combined } \\
\text { with phonological } \\
\text { awareness } \\
\text { intervention }\end{array}$ & $n=12(3 ; 0-3 ; 11)$ & $\begin{array}{l}\text { Individual and group } \\
\text { intervention delivered } \\
\text { by an SLT in a } \\
\text { university clinic. }\end{array}$ & $\begin{array}{l}\text { Each child received } 3 \text { or } 4 \\
\text { blocks of intervention. } \\
\text { Each block lasted } 4-6 \\
\text { weeks and comprised } 2 \\
\times 45 \text {-min sessions per } \\
\text { week. In total, children } \\
\text { received between } 16 \\
\text { and } 34 \text { sessions before } \\
\text { school entry (average } \\
25.5 \text { ), and 10-12 hours } \\
\text { of intervention } \\
\text { following school entry. }\end{array}$ & $5 / 7$ \\
\hline
\end{tabular}




\begin{tabular}{|c|c|c|c|c|c|c|}
\hline Reference & Research design & $\begin{array}{l}\text { Intervention } \\
\text { approach }\end{array}$ & $\begin{array}{c}\text { Participant } \\
\text { number and age } \\
\text { (years;months) }\end{array}$ & $\begin{array}{c}\text { Summary of service } \\
\text { delivery }^{\mathrm{a}}\end{array}$ & $\begin{array}{c}\text { Summary of intervention } \\
\text { intensity }\end{array}$ & $\begin{array}{l}\text { Reporting of } \\
\text { intervention } \\
\text { intensity }^{b}\end{array}$ \\
\hline $\begin{array}{l}\text { Girolametto, Pearce and } \\
\text { Weitzman (1997) }\end{array}$ & RCT & $\begin{array}{l}\text { Lexical intervention } \\
\text { based on Hanen } \\
\text { principles }\end{array}$ & $n=25(1 ; 11-2 ; 9)$ & $\begin{array}{l}\text { Group parent-training } \\
\text { sessions delivered by an } \\
\text { SLT at a clinic, with } \\
\text { most intervention } \\
\text { delivered at home by } \\
\text { parents. The SLT also } \\
\text { conducted individual } \\
\text { home-based sessions } \\
\text { with each participant. }\end{array}$ & $\begin{array}{l}8 \times 2.5 \text {-hour group parent } \\
\text { training sessions and } 3 \\
\times \text { home-visits over a } \\
\text { total of } 11 \text { weeks. } \\
\text { Sessions were } \\
\text { conducted } \\
\text { approximately weekly. }\end{array}$ & $5 / 7$ \\
\hline $\begin{array}{l}\text { Glaspey and Macleod } \\
\quad(2010)\end{array}$ & Case study & Modified cycles & $n=1$ 'G' $(3 ; 5)$ & $\begin{array}{l}\text { Individual intervention } \\
\text { delivered by an SLT at } \\
\text { school. }\end{array}$ & $\begin{array}{l}2 \times 50 \text {-min sessions per } \\
\text { week for a total of } 32 \\
\text { sessions delivered over } \\
\text { a 6-month period. }\end{array}$ & $5 / 7$ \\
\hline $\begin{array}{l}\text { Glaspey and Stoel- } \\
\text { Gammon (2005) }\end{array}$ & Case study & Cycles & $n=1$ 'Ann' $(3 ; 7)$ & $\begin{array}{l}\text { Individual intervention } \\
\text { delivered by an SLT. }\end{array}$ & $\begin{array}{l}2 \times 50 \text {-min sessions per } \\
\text { week for a total of } 8 \\
\text { weeks. }\end{array}$ & $4 / 7$ \\
\hline $\begin{array}{l}\text { Glaspey and Stoel- } \\
\text { Gammon (2007) }\end{array}$ & Case study & Cycles & $n=1$ 'Mark' (4) & $\begin{array}{l}\text { Individual intervention by } \\
\text { an SLT. }\end{array}$ & $\begin{array}{l}2 \text { sessions per week over a } \\
\text { 6-month period. }\end{array}$ & $3 / 7$ \\
\hline $\begin{array}{l}\text { Glogowska, Roulstone, } \\
\text { Enderby and Peters } \\
(2000)\end{array}$ & $\mathrm{RCT}$ & $\begin{array}{l}\text { Community-based } \\
\text { speech and } \\
\text { language therapy }\end{array}$ & $\begin{array}{l}n=159 \\
\text { Experimental group: } \\
\quad n=71(1 ; 6-3 ; 9) \\
\text { Control group: } n= \\
\quad 88(2 ; 0-3 ; 6)\end{array}$ & $\begin{array}{l}\text { Individual intervention } \\
\text { delivered by an SLT in } \\
\text { a community clinic. }\end{array}$ & $\begin{array}{l}\text { Average of } 8.1 \text { sessions, } \\
\text { totalling an average of } \\
6.2 \text { hours of } \\
\text { intervention delivered } \\
\text { over a mean of } 8.4\end{array}$ & $5 / 7$ \\
\hline Goldstein (1996) & Case study & $\begin{array}{l}\text { Generic phonological } \\
\text { approach }\end{array}$ & $n=1$ 'Mario' $(3 ; 5)$ & $\begin{array}{l}\text { Individual intervention } \\
\text { delivered by an SLT. }\end{array}$ & $\begin{array}{l}\text { Average of } 2 \text { sessions per } \\
\text { week for } 4 \text { months. }\end{array}$ & $3 / 7$ \\
\hline $\begin{array}{l}\text { Gordon-Brannan, } \\
\text { Hodson and Wynne } \\
\text { (1992) }\end{array}$ & Case study & Cycles & $n=1$ 'Luke' $(4 ; 6)$ & $\begin{array}{l}\text { Individual intervention } \\
\text { delivered by an SLT in } \\
\text { a university clinic. }\end{array}$ & $\begin{array}{l}1 \times 75 \text {-min session per } \\
\text { week for a total of } 66 \\
\text { sessions delivered over } \\
\text { approx. } 66 \text { weeks. }\end{array}$ & $5 / 7$ \\
\hline
\end{tabular}




\begin{tabular}{|c|c|c|c|c|c|c|}
\hline Reference & Research design & $\begin{array}{l}\text { Intervention } \\
\text { approach }\end{array}$ & $\begin{array}{c}\text { Participant } \\
\text { number and age } \\
\text { (years;months) }\end{array}$ & $\begin{array}{c}\text { Summary of service } \\
\text { delivery }^{\mathbf{a}}\end{array}$ & $\begin{array}{l}\text { Summary of intervention } \\
\text { intensity }\end{array}$ & $\begin{array}{c}\text { Reporting of } \\
\text { intervention } \\
\text { intensity }^{\mathbf{b}}\end{array}$ \\
\hline $\begin{array}{l}\text { Grawburg and Rvachew } \\
\text { (2007) }\end{array}$ & $\begin{array}{l}\text { Within subjects } \\
\text { design }\end{array}$ & $\begin{array}{l}\text { Phonological } \\
\text { awareness } \\
\text { intervention } \\
\text { combined with } \\
\text { speech perception } \\
\text { training (SAILS) }\end{array}$ & $\begin{array}{l}n=30\left(\mathrm{M}_{\text {age }}=56.7-\right. \\
57.6 \text { months }) \\
\text { Experimental group: } \\
\quad n=10\left(\mathrm{M}_{\mathrm{age}}=\right. \\
56.7 \text { months })\end{array}$ & $\begin{array}{l}\text { Intervention delivered by a } \\
\text { student SLT in a } \\
\text { university clinic. }\end{array}$ & $\begin{array}{l}1 \times 45-60 \text { min session per } \\
\text { week for a total of } 8 \\
\text { sessions delivered over } \\
8 \text { weeks. A total of } 6-8 \\
\text { hours of intervention } \\
\text { was delivered. }\end{array}$ & $5 / 7$ \\
\hline $\begin{array}{l}\text { Grunwell and Dive } \\
\text { (1988) }\end{array}$ & Case study & $\begin{array}{l}\text { Minimal pairs } \\
\text { including } \\
\text { elements of } \\
\text { traditional } \\
\text { articulation } \\
\text { intervention. }\end{array}$ & $\begin{array}{l}n=2 \\
\text { 'L' }(6 ; 0) \\
\text { 'H' }(8 ; 0)\end{array}$ & $\begin{array}{l}\text { Intensive residential } \\
\text { program, including } \\
\text { individual and group } \\
\text { intervention delivered } \\
\text { by an SLT in a hospital } \\
\text { clinic. }\end{array}$ & $\begin{array}{l}5 \times 4.5 \text {-hour sessions of } \\
\text { intervention per week } \\
\text { for a total of } 10 \text { sessions } \\
\text { over } 2 \text { weeks. }\end{array}$ & $5 / 7$ \\
\hline $\begin{array}{l}\text { Grunwell and Russell } \\
\quad(1990)\end{array}$ & Case study & Minimal pairs & $n=1$ 'Neil' $(4 ; 3)$ & $\begin{array}{l}\text { Individual intervention } \\
\text { delivered by an SLT in } \\
\text { a hospital clinic. }\end{array}$ & $\begin{array}{l}4 \times 30-40 \text {-min sessions } \\
\text { per week for } 12 \text { weeks, } \\
\text { for approx. } 48 \text { sessions } \\
\text { in total. }\end{array}$ & $5 / 7$ \\
\hline $\begin{array}{l}\text { Grunwell, Yavas, Russell } \\
\text { and Le Maistre (1988) }\end{array}$ & Case study & Minimal pairs & $n=1$ 'N' $(5 ; 0)$ & $\begin{array}{l}\text { Small group intervention } \\
\text { with an SLT. }\end{array}$ & $\begin{array}{l}\text { Weekly sessions delivered } \\
\text { over a period of } 4 \\
\text { months. }\end{array}$ & $3 / 7$ \\
\hline $\begin{array}{l}\text { Harbers, Paden and Halle } \\
\quad \text { (1999) }\end{array}$ & $\begin{array}{l}\text { MBD across } \\
\text { participants }\end{array}$ & $\begin{array}{l}\text { Metaphon combined } \\
\text { with cycles }\end{array}$ & $n=4(3 ; 5-4 ; 2)$ & $\begin{array}{l}\text { Individual intervention } \\
\text { delivered by an SLT in } \\
\text { a university clinic. }\end{array}$ & $\begin{array}{l}2 \times 45 \text {-min sessions per } \\
\text { week for } 6-9 \text { months. } \\
\text { Total duration of } \\
\text { intervention (in } \\
\text { sessions) reported but } \\
\text { unclear. }\end{array}$ & $5 / 7$ \\
\hline $\begin{array}{l}\text { Hart and Gonzalez } \\
\text { (2010) }\end{array}$ & $\begin{array}{l}\text { MPD across } \\
\text { participants }\end{array}$ & $\begin{array}{l}\text { Communication- } \\
\text { centred } \\
\text { intervention using } \\
\text { storybooks }\end{array}$ & $n=3(3 ; 7-4 ; 11)$ & $\begin{array}{l}\text { Individual intervention } \\
\text { delivered by an SLT in } \\
\text { a preschool. }\end{array}$ & $\begin{array}{l}2 \times 30 \text {-min sessions per } \\
\text { week for a total of } 12 \\
\text { sessions over } 6 \text { weeks. } \\
\text { A total of } 10 \text { perception } \\
\text { trials and } 20-30 \\
\text { production trials were } \\
\text { provided in each } \\
\text { session. }\end{array}$ & $6 / 7$ \\
\hline
\end{tabular}




\begin{tabular}{|c|c|c|c|c|c|c|}
\hline Reference & Research design & $\begin{array}{l}\text { Intervention } \\
\text { approach }\end{array}$ & $\begin{array}{c}\text { Participant } \\
\text { number and age } \\
\text { (years;months) }\end{array}$ & $\begin{array}{c}\text { Summary of service } \\
\text { delivery }\end{array}$ & $\begin{array}{l}\text { Summary of intervention } \\
\text { intensity }\end{array}$ & $\begin{array}{l}\text { Reporting of } \\
\text { intervention } \\
\text { intensity }^{b}\end{array}$ \\
\hline $\begin{array}{l}\text { Herman, Ford, Thomas, } \\
\text { Oyebade, Bennett and } \\
\text { Dodd (2015) }\end{array}$ & Case study & Core vocabulary & $n=4(9 ; 0-11 ; 3)$ & $\begin{array}{l}\text { Individual intervention } \\
\text { delivered by an SLT in } \\
\text { a university clinic. }\end{array}$ & $\begin{array}{l}2 \times 45 \text {-min sessions per } \\
\text { week for } 16 \text { sessions } \\
\text { over } 8 \text { weeks. A } \\
\text { production dose of } 200 \\
\text { was provided in the } \\
\text { second session each } \\
\text { week. }\end{array}$ & $6 / 7$ \\
\hline $\begin{array}{l}\text { Hesketh, Adams, } \\
\text { Nightingale and Hall } \\
(2000)\end{array}$ & Non-RCT & $\begin{array}{l}\text { Metaphonological } \\
\text { intervention } \\
\text { compared with } \\
\text { traditional } \\
\text { articulation }\end{array}$ & $\begin{array}{l}n=61(3 ; 6-5 ; 0) \text { and } \\
59 \text { controls }\end{array}$ & $\begin{array}{l}\text { Individual intervention } \\
\text { delivered by an SLT in } \\
\text { a university clinic. }\end{array}$ & $\begin{array}{l}1 \times \text { session per week for a } \\
\text { total of } 10 \text { sessions over } \\
10 \text { weeks. }\end{array}$ & $4 / 7$ \\
\hline $\begin{array}{l}\text { Hesketh, Dima and } \\
\text { Nelson (2007) }\end{array}$ & RCT & $\begin{array}{l}\text { Phonological } \\
\text { awareness } \\
\text { intervention }\end{array}$ & $n=42(4 ; 0-4 ; 6)$ & $\begin{array}{l}\text { Individual intervention } \\
\text { delivered by an SLT in } \\
\text { homes or schools. }\end{array}$ & $\begin{array}{l}2-3 \times 30 \text {-min sessions per } \\
\text { week for a total of } 20 \\
\text { sessions delivered over } \\
\text { a maximum of } 10 \\
\text { weeks. }\end{array}$ & $5 / 7$ \\
\hline Hodson (1983) & Case study & Cycles & $n=1$ 'Candi' $(3 ; 11)$ & $\begin{array}{l}\text { Individual intervention } \\
\text { delivered by an SLT } \\
\text { student in a university } \\
\text { clinic. }\end{array}$ & $\begin{array}{l}1 \times \text { session per week for a } \\
\text { total of } 45 \text { sessions over } \\
18 \text { months. Sessions } \\
\text { included a perceptual } \\
\text { dose of approx. } 30 . \\
\text { Production dose } \\
\text { included but not } \\
\text { reported. Intervention } \\
\text { was provided from } \\
\text { referral to discharge. }\end{array}$ & $4 / 7$ \\
\hline
\end{tabular}




\begin{tabular}{|c|c|c|c|c|c|c|}
\hline Reference & Research design & $\begin{array}{l}\text { Intervention } \\
\text { approach }\end{array}$ & $\begin{array}{c}\text { Participant } \\
\text { number and age } \\
\text { (years;months) }\end{array}$ & $\begin{array}{c}\text { Summary of service } \\
\text { delivery }^{\mathrm{a}}\end{array}$ & $\begin{array}{l}\text { Summary of intervention } \\
\text { intensity }\end{array}$ & $\begin{array}{l}\text { Reporting of } \\
\text { intervention } \\
\text { intensity }^{b}\end{array}$ \\
\hline $\begin{array}{l}\text { Hodson, Chin, Redmond } \\
\text { and Simpson (1983) }\end{array}$ & Case study & Cycles & $n=1$ 'Tim’ (5) & $\begin{array}{l}\text { Individual intervention } \\
\text { delivered by an SLT in } \\
\text { a university clinic and } \\
\text { by a school-based SLT } \\
\text { at school. }\end{array}$ & $\begin{array}{l}1 \times 60-90 \text {-min session per } \\
\text { week at the university } \\
\text { clinic }(36 \text { sessions in } \\
\text { total) plus } 2 \times 20 \text {-min } \\
\text { sessions per week at } \\
\text { school. Sessions } \\
\text { included a perceptual } \\
\text { dose of approx. } 30 \\
\text { trials. Production dose } \\
\text { included but not } \\
\text { reported. In total, } 65 \\
\text { hours of intervention } \\
\text { over } 13 \text { months. }\end{array}$ & $5 / 7$ \\
\hline $\begin{array}{l}\text { Hodson, Nonomura and } \\
\text { Zappia (1989) }\end{array}$ & Case study & Cycles & $n=1$ 'Lisa' $(5 ; 0)$ & $\begin{array}{l}\text { Individual intervention } \\
\text { delivered by an SLT in } \\
\text { a university clinic. }\end{array}$ & $\begin{array}{c}1 \times 90 \text {-min session per } \\
\text { week delivered over } 3 \\
\text { university semesters. }\end{array}$ & $4 / 7$ \\
\hline $\begin{array}{l}\text { Hoffman, Norris and } \\
\text { Monjure (1990) }\end{array}$ & Case study & $\begin{array}{l}\text { Minimal pairs and } \\
\text { whole language }\end{array}$ & $\begin{array}{c}n=2(4 ; 1, \text { two of a } \\
\text { set of triplets })\end{array}$ & $\begin{array}{l}\text { Individual intervention } \\
\text { delivered by an SLT } \\
\text { student in a university } \\
\text { clinic. }\end{array}$ & $\begin{array}{l}3 \times 50 \text {-min sessions per } \\
\text { week for } 6 \text { weeks. }\end{array}$ & $4 / 7$ \\
\hline Holm and Dodd (1999) & Case study & Core vocabulary & $n=1$ 'HK' $(4 ; 6)$ & $\begin{array}{l}\text { Individual intervention } \\
\text { delivered by an SLT at } \\
\text { home and at school. }\end{array}$ & $\begin{array}{l}2 \times 30 \text {-min sessions per } \\
\text { week for } 8 \text { weeks }(16 \\
\text { sessions in total) }\end{array}$ & $5 / 7$ \\
\hline \multirow[t]{2}{*}{ Holm and Dodd (2001) } & \multirow[t]{2}{*}{ Case study } & \multirow{2}{*}{$\begin{array}{l}\text { Core vocabulary, } \\
\text { traditional } \\
\text { articulation } \\
\text { therapy, and } \\
\text { minimal pairs }\end{array}$} & \multirow[t]{2}{*}{$n=2(4 ; 8-5 ; 2)$} & $\begin{array}{l}\text { 'Hafis' (age 4;8): } \\
\text { Individual intervention } \\
\text { delivered by an SLT. }\end{array}$ & $\begin{array}{l}\text { 'Hafis': } 2 \times 30 \text {-min } \\
\text { sessions per week for } 8 \\
\text { weeks for a total of } 16 \\
\text { sessions. }\end{array}$ & $5 / 7$ \\
\hline & & & & $\begin{array}{l}\text { 'Jason' (age } 5 ; 2 \text { ): } \\
\text { Individual intervention } \\
\text { delivered by at SLT at } \\
\text { childcare. }\end{array}$ & $\begin{array}{l}\text { ‘Jason': } 2 \times 20 \text {-min } \\
\text { sessions per week for } 7 \\
\text { weeks, followed by } 1 \times \\
45 \text {-min session per } \\
\text { week for } 8 \text { weeks. Total } \\
\text { duration of intervention } \\
\text { was } 22 \text { sessions over } 15 \\
\text { weeks. }\end{array}$ & $5 / 7$ \\
\hline
\end{tabular}




\begin{tabular}{|c|c|c|c|c|c|c|}
\hline Reference & Research design & $\begin{array}{l}\text { Intervention } \\
\text { approach }\end{array}$ & $\begin{array}{c}\text { Participant } \\
\text { number and age } \\
\text { (years;months) }\end{array}$ & $\begin{array}{c}\text { Summary of service } \\
\text { delivery }^{\mathrm{a}}\end{array}$ & $\begin{array}{l}\text { Summary of intervention } \\
\text { intensity }\end{array}$ & $\begin{array}{l}\text { Reporting of } \\
\text { intervention } \\
\text { intensity }^{b}\end{array}$ \\
\hline $\begin{array}{l}\text { Holm, Dodd and Ozanne } \\
\text { (1997) }\end{array}$ & Case study & $\begin{array}{l}\text { Traditional } \\
\text { articulation } \\
\text { therapy and } \\
\text { minimal pairs }\end{array}$ & $n=1$ 'JL' $(5 ; 2)$ & $\begin{array}{l}\text { Articulation therapy: } \\
\text { Individual intervention } \\
\text { delivered by an SLT at } \\
\text { childcare. } \\
\text { Minimal pairs: Individual } \\
\text { intervention delivered } \\
\text { by an SLT at au } \\
\text { university clinic or at } \\
\text { home. }\end{array}$ & $\begin{array}{l}\text { Articulation therapy: } 2 \times \\
20 \text {-min sessions per } \\
\text { week for } 7 \text { weeks (total } \\
14 \text { sessions). } \\
\text { Minimal pairs: } 1 \times 45 \text {-min } \\
\text { session per week for } 8 \\
\text { weeks (total } 8 \text { sessions). }\end{array}$ & $5 / 7$ \\
\hline Jarvis (1989) & Case study & Metaphon & $n=1$ 'Luke' $(4 ; 9)$ & $\begin{array}{l}\text { Individual intervention } \\
\text { delivered by a specialist } \\
\text { teacher (teacher of the } \\
\text { deaf) at school. }\end{array}$ & $\begin{array}{l}\text { 20-min sessions, initially } \\
\text { delivered } 3 \times \text { per week } \\
\text { then reducing to } 2 \times \text { per } \\
\text { week and then to } 1 \times \\
\text { per week. Intervention } \\
\text { delivered over an } \\
\text { academic year }(10 \\
\text { months). Intervention } \\
\text { was provided from } \\
\text { referral to discharge. }\end{array}$ & $4 / 7$ \\
\hline $\begin{array}{l}\text { Keske-Soares, } \\
\text { Brancalioni, Marini, } \\
\text { Pagliarin and Ceron } \\
\text { (2008) }\end{array}$ & $\begin{array}{l}\text { Between groups } \\
\text { design }\end{array}$ & $\begin{array}{l}\text { ABAB withdrawal } \\
\text { and multiple } \\
\text { probes compared } \\
\text { with modified } \\
\text { maximal } \\
\text { oppositions } \\
\text { compared with } \\
\text { modified cycles }\end{array}$ & $n=66(4 ; 4-8 ; 2)$ & $\begin{array}{l}\text { Delivered by a student } \\
\text { SLT at a university } \\
\text { clinic. }\end{array}$ & 15-25 sessions delivered. & $2 / 7$ \\
\hline
\end{tabular}




\begin{tabular}{|c|c|c|c|c|c|c|}
\hline Reference & Research design & $\begin{array}{l}\text { Intervention } \\
\text { approach }\end{array}$ & $\begin{array}{c}\text { Participant } \\
\text { number and age } \\
\text { (years;months) }\end{array}$ & $\begin{array}{c}\text { Summary of service } \\
\text { delivery }^{\mathrm{a}}\end{array}$ & $\begin{array}{l}\text { Summary of intervention } \\
\text { intensity }^{\mathrm{a}}\end{array}$ & $\begin{array}{l}\text { Reporting of } \\
\text { intervention } \\
\text { intensity }^{b}\end{array}$ \\
\hline Klein (1996) & $\begin{array}{l}\text { Retrospective } \\
\text { between groups } \\
\text { comparison }\end{array}$ & $\begin{array}{l}\text { Imagery approach } \\
\text { compared with } \\
\text { traditional } \\
\text { articulation } \\
\text { therapy }\end{array}$ & $n=36(3 ; 0-5 ; 10)$ & $\begin{array}{l}\text { Individual intervention } \\
\text { delivered by SLT } \\
\text { students at a university } \\
\text { clinic. }\end{array}$ & $\begin{array}{l}2-3 \times 50 \text {-min sessions per } \\
\text { week. For the } \\
\text { phonological group, the } \\
\text { total duration of } \\
\text { intervention was an } \\
\text { average of } 81.88 \\
\text { sessions over } 13.47 \\
\text { months. For the } \\
\text { articulation group, an } \\
\text { average of } 100.95 \\
\text { sessions were delivered } \\
\text { over } 22.32 \text { months. } \\
\text { Intervention was } \\
\text { provided from referral } \\
\text { to discharge. }\end{array}$ & $5 / 7$ \\
\hline \multirow[t]{2}{*}{$\begin{array}{l}\text { Lancaster, Keusch, } \\
\text { Levin, Pring and } \\
\text { Martin (2010) }\end{array}$} & Study 1: RCT & $\begin{array}{l}\text { Study 1: Community } \\
\text { based intervention }\end{array}$ & $\begin{array}{l}\text { Study } 1: n=12 \\
\quad(3 ; 4-5 ; 10)\end{array}$ & $\begin{array}{l}\text { Study 1: Individual } \\
\text { intervention delivered } \\
\text { by an SLT in a } \\
\text { community clinic. }\end{array}$ & $\begin{array}{l}\text { Study } 1: 1 \times 30 \text {-min } \\
\text { session per week for a } \\
\text { total of } 8 \text { sessions over } \\
3 \text { months. }\end{array}$ & $5 / 7$ \\
\hline & Study 2: RCT & $\begin{array}{l}\text { Study 2: Community } \\
\text { based intervention }\end{array}$ & $\begin{array}{l}\text { Study } 2: n=15 \\
\quad(3 ; 4-4 ; 5)\end{array}$ & $\begin{array}{l}\text { Study 2: Treated group: } \\
\text { Individual intervention } \\
\text { delivered by an SLT in } \\
\text { a community clinic. } \\
\text { Parent group: Parent- } \\
\text { delivered intervention at } \\
\text { home. }\end{array}$ & $\begin{array}{l}\text { Study 2: Treated group } 1 \times \\
\text { 30-min session per } \\
\text { week for a total of } 15 \\
\text { sessions over } 6 \text { months. } \\
\text { Parent group received } 1 \\
\times 2 \text {-hour group parent } \\
\text { training session with } \\
\text { follow-up meetings } \\
\text { every } 6 \text { weeks over a } \\
\text { period of } 6 \text { months. }\end{array}$ & $5 / 7$ \\
\hline $\begin{array}{l}\text { Law, Garrett and Nye } \\
\quad \text { (2004) }\end{array}$ & $\begin{array}{l}\text { Systematic review } \\
\text { and meta- } \\
\text { analysis }\end{array}$ & $\begin{array}{l}\text { A range of } \\
\text { phonological and } \\
\text { articulation-based } \\
\text { approaches }\end{array}$ & $\begin{array}{l}13 \text { articles spanning } \\
25 \text { years } \\
\text { (including } 6 \\
\text { phonological } \\
\text { intervention } \\
\text { studies) }\end{array}$ & $\begin{array}{l}\text { Varied across included } \\
\text { studies }\end{array}$ & $\begin{array}{l}\text { Varied across included } \\
\text { studies }\end{array}$ & - \\
\hline
\end{tabular}




\begin{tabular}{|c|c|c|c|c|c|c|}
\hline Reference & Research design & $\begin{array}{l}\text { Intervention } \\
\text { approach }\end{array}$ & $\begin{array}{c}\text { Participant } \\
\text { number and age } \\
\text { (years;months) }\end{array}$ & $\begin{array}{c}\text { Summary of service } \\
\text { delivery }^{\mathrm{a}}\end{array}$ & $\begin{array}{l}\text { Summary of intervention } \\
\text { intensity }^{\mathrm{a}}\end{array}$ & $\begin{array}{c}\text { Reporting of } \\
\text { intervention } \\
\text { intensity }\end{array}$ \\
\hline Leahy and Dodd (1987) & Case study & Minimal pairs & $n=1$ 'AJ' $(3 ; 5)$ & $\begin{array}{l}\text { Individual intervention } \\
\text { delivered by an SLT at a } \\
\text { university clinic, } \\
\text { supported by parent at } \\
\text { home. }\end{array}$ & $\begin{array}{l}\text { Fortnightly sessions, for a } \\
\text { total of } 13 \text { sessions } \\
\text { delivered over } 6 \\
\text { months. Of the } 13 \\
\text { sessions, } 5 \text { were } \\
\text { assessment and } 8 \text { were } \\
\text { treatment. }\end{array}$ & $4 / 7$ \\
\hline $\begin{array}{l}\text { Leite, Wertzner, } \\
\text { Goncalves, Magliaro } \\
\text { and Matas (2014) }\end{array}$ & Non-RCT & Modified cycles & $n=23\left(\mathrm{M}_{\mathrm{age}}=8 ; 10\right)$ & $\begin{array}{l}\text { Individual intervention } \\
\text { delivered by an SLT in } \\
\text { a university clinic. }\end{array}$ & $\begin{array}{l}1 \times 45 \text {-min session per } \\
\text { week for a total of } 12 \\
\text { sessions delivered over } \\
12 \text { weeks. }\end{array}$ & $5 / 7$ \\
\hline $\begin{array}{l}\text { Lousada, Jesus, Capelas, } \\
\text { Margaca, Simoes, } \\
\text { Valente, Hall and } \\
\text { Joffe (2013) }\end{array}$ & $\mathrm{RCT}$ & $\begin{array}{l}\text { Generic phonological } \\
\text { approach } \\
\text { combined with } \\
\text { phonological } \\
\text { awareness } \\
\text { compared with } \\
\text { traditional } \\
\text { articulation } \\
\text { intervention }\end{array}$ & $n=14(4 ; 0-6 ; 7)$ & $\begin{array}{l}\text { Individual intervention } \\
\text { delivered by an SLT in } \\
\text { a university clinic. }\end{array}$ & $\begin{array}{l}1 \times 45 \text {-min session per } \\
\text { week for a total of } 25 \\
\text { sessions over } 25 \text { weeks. } \\
\text { Sessions were divided } \\
\text { into } 3 \text { blocks (of } 9,8 \\
\text { and } 8 \text { weeks in } \\
\text { duration) without } \\
\text { breaks. }\end{array}$ & $5 / 7$ \\
\hline $\begin{array}{l}\text { Lousada, Jesus, Hall and } \\
\text { Joffe (2014) }\end{array}$ & RCT & $\begin{array}{l}\text { Generic phonological } \\
\text { approach } \\
\text { combined with } \\
\text { phonological } \\
\text { awareness } \\
\text { compared with } \\
\text { traditional } \\
\text { articulation } \\
\text { intervention }\end{array}$ & $n=14(4 ; 0-6 ; 7)$ & $\begin{array}{l}\text { Individual intervention } \\
\text { delivered by an SLT in } \\
\text { a university clinic. }\end{array}$ & $\begin{array}{l}1 \times 45 \text {-min session per } \\
\text { week for a total of } 25 \\
\text { sessions over } 25 \text { weeks. } \\
\text { Sessions were divided } \\
\text { into } 3 \text { blocks (of } 9,8 \\
\text { and } 8 \text { weeks in } \\
\text { duration) without } \\
\text { breaks. }\end{array}$ & $5 / 7$ \\
\hline $\begin{array}{l}\text { MacLeod and Glaspey } \\
\quad \text { (2014) }\end{array}$ & Case study & Cycles & $n=3(3 ; 0)$ & $\begin{array}{l}\text { Individual intervention } \\
\text { delivered by an SLT in } \\
\text { a university clinic. }\end{array}$ & $\begin{array}{l}2 \times \text { sessions per week for a } \\
\text { total of } 16 \text { sessions over } \\
\text { approx. } 8 \text { weeks. }\end{array}$ & $4 / 7$ \\
\hline
\end{tabular}




\begin{tabular}{|c|c|c|c|c|c|c|}
\hline Reference & Research design & $\begin{array}{l}\text { Intervention } \\
\text { approach }\end{array}$ & $\begin{array}{c}\text { Participant } \\
\text { number and age } \\
\text { (years;months) }\end{array}$ & $\begin{array}{c}\text { Summary of service } \\
\text { delivery }^{\mathrm{a}}\end{array}$ & $\begin{array}{c}\text { Summary of intervention } \\
\text { intensity }\end{array}$ & $\begin{array}{c}\text { Reporting of } \\
\text { intervention } \\
\text { intensity }\end{array}$ \\
\hline $\begin{array}{l}\text { Major and Bernhardt } \\
\text { (1998) }\end{array}$ & $\begin{array}{l}\text { Between groups } \\
\text { design }\end{array}$ & $\begin{array}{l}\text { Nonlinear } \\
\text { intervention with } \\
\text { or without } \\
\text { metaphonological } \\
\text { intervention }\end{array}$ & $n=19(3 ; 0-4 ; 11)$ & $\begin{array}{l}\text { Individual intervention } \\
\text { delivered by an SLT in } \\
\text { a community clinic. }\end{array}$ & $\begin{array}{l}3 \times \text { sessions per week for a } \\
\text { total of } 48 \text { sessions over } \\
\text { three treatment blocks } \\
\text { of } 12 \text { or } 18 \text { sessions in } \\
\text { duration. One child } \\
\text { ('Kendra') received } \\
\text { intervention } 1 \times \text { weekly } \\
\text { for two } 8 \text {-week blocks. } \\
\text { Intervention was } \\
\text { delivered over } 5-10 \\
\text { months. }\end{array}$ & $4 / 7$ \\
\hline $\begin{array}{l}\text { Masterson and Daniels } \\
\quad(1991)\end{array}$ & Case study & $\begin{array}{l}\text { Minimal pairs and } \\
\text { motoric } \\
\text { automatization }\end{array}$ & $n=1$ 'C $C^{\prime}(3 ; 8)$ & $\begin{array}{l}\text { Individual intervention } \\
\text { delivered by an SLT } \\
\text { student in a university } \\
\text { clinic. }\end{array}$ & $\begin{array}{l}2 \times 50 \text {-min sessions per } \\
\text { week delivered over } 4 \\
\text { university semesters }(25 \\
\text { months). Intervention } \\
\text { was provided from } \\
\text { referral to discharge. }\end{array}$ & $4 / 7$ \\
\hline $\begin{array}{l}\text { McIntosh and Dodd } \\
\text { (2008) }\end{array}$ & Case study & Core vocabulary & $n=3(3 ; 9-4 ; 3)$ & $\begin{array}{l}\text { Individual intervention } \\
\text { delivered by an SLT in } \\
\text { a hospital clinic. }\end{array}$ & $\begin{array}{l}2 \times 30-40-\text { min sessions } \\
\text { per week for between } \\
12 \text { and } 38 \text { sessions } \\
\text { delivered over approx. } \\
6-19 \text { weeks. The second } \\
\text { session of each week } \\
\text { contained } 20-240 \\
\text { production trials. }\end{array}$ & $6 / 7$ \\
\hline
\end{tabular}




\begin{tabular}{|c|c|c|c|c|c|c|}
\hline Reference & Research design & $\begin{array}{l}\text { Intervention } \\
\text { approach }\end{array}$ & $\begin{array}{c}\text { Participant } \\
\text { number and age } \\
\text { (years;months) }\end{array}$ & $\begin{array}{c}\text { Summary of service } \\
\text { delivery }^{\mathbf{a}}\end{array}$ & $\begin{array}{c}\text { Summary of intervention } \\
\text { intensity }\end{array}$ & $\begin{array}{l}\text { Reporting of } \\
\text { intervention } \\
\text { intensity }^{\mathbf{b}}\end{array}$ \\
\hline $\begin{array}{l}\text { McKean, Phillips and } \\
\text { Thompson (2012) }\end{array}$ & RCT & $\begin{array}{l}\text { Generic phonological } \\
\text { approach using } \\
\text { elements of } \\
\text { family-centred } \\
\text { practice }\end{array}$ & $n=20(3 ; 3-4 ; 10)$ & $\begin{array}{l}\text { Usual practice group: } \\
\text { Individual intervention } \\
\text { and a group parent } \\
\text { training session } \\
\text { delivered by an SLT in } \\
\text { a community clinic, } \\
\text { plus parent-delivered } \\
\text { intervention at home. } \\
\text { Family-centred practice } \\
\text { group: Individual } \\
\text { intervention and a group } \\
\text { parent training delivered } \\
\text { by an SLT in a } \\
\text { community clinic and at } \\
\text { home, plus parent- } \\
\text { delivered intervention at } \\
\text { home. }\end{array}$ & $\begin{array}{l}1 \times 45 \text {-min session per } \\
\text { week for a total of } 9 \\
\text { SLT-delivered } \\
\text { intervention sessions } \\
\text { over } 14 \text { weeks. }\end{array}$ & $5 / 7$ \\
\hline $\begin{array}{l}\text { Mecrow, Beckwith and } \\
\text { Klee (2010) }\end{array}$ & $\begin{array}{l}\text { Between groups } \\
\text { design }\end{array}$ & $\begin{array}{l}\text { Generic phonological } \\
\text { approach }\end{array}$ & $n=35(4 ; 2-6 ; 10)$ & $\begin{array}{l}\text { Individual intervention } \\
\text { delivered by an SLT } \\
\text { assistant at school. }\end{array}$ & $\begin{array}{l}\text { Average of } 4 \times 45-60-\min \\
\text { sessions per week for } 10 \\
\text { weeks for an average of } \\
39 \text { sessions. }\end{array}$ & $5 / 7$ \\
\hline $\begin{array}{l}\text { Mezzomo, Mota, Keske- } \\
\text { Soares, Ceron and } \\
\text { Dias (2014) }\end{array}$ & Case study & $\begin{array}{l}\text { ABAB withdrawal } \\
\text { and multiple } \\
\text { probes compared } \\
\text { with minimal pairs } \\
\text { compared with } \\
\text { maximal } \\
\text { oppositions }\end{array}$ & $n=5(5 ; 0-6 ; 11)$ & $\begin{array}{l}\text { Individual intervention } \\
\text { delivered by an SLT at a } \\
\text { university clinic. }\end{array}$ & $\begin{array}{l}\text { A total of } 9 \text { to } 25 \text { sessions } \\
\text { provided. }\end{array}$ & $2 / 7$ \\
\hline Miccio and Elbert (1996) & Case study & $\begin{array}{l}\text { Stimulability } \\
\text { intervention }\end{array}$ & $n=1$ 'Stacy' $(3 ; 4)$ & $\begin{array}{l}\text { Individual intervention } \\
\text { delivered by an SLT. }\end{array}$ & $\begin{array}{l}2 \times 45 \text {-min sessions per } \\
\text { week for a total of } 12 \\
\text { sessions delivered over } \\
6 \text { weeks. }\end{array}$ & $5 / 7$ \\
\hline
\end{tabular}




\begin{tabular}{|c|c|c|c|c|c|c|}
\hline Reference & Research design & $\begin{array}{l}\text { Intervention } \\
\text { approach }\end{array}$ & $\begin{array}{c}\text { Participant } \\
\text { number and age } \\
\text { (years;months) }\end{array}$ & $\begin{array}{c}\text { Summary of service } \\
\text { delivery }^{\mathrm{a}}\end{array}$ & $\begin{array}{l}\text { Summary of intervention } \\
\text { intensity }\end{array}$ & $\begin{array}{c}\text { Reporting of } \\
\text { intervention } \\
\text { intensity }^{\mathbf{b}}\end{array}$ \\
\hline $\begin{array}{l}\text { Miccio, Elbert and } \\
\text { Forrest (1999) }\end{array}$ & $\begin{array}{l}\text { MBD across } \\
\text { participants }\end{array}$ & Minimal pairs & $n=4(3 ; 10-5 ; 7)$ & $\begin{array}{l}\text { Individual intervention } \\
\text { delivered by an SLT in } \\
\text { a university clinic. }\end{array}$ & $\begin{array}{l}2 \times 45 \text {-min sessions per } \\
\text { week for a total of } 20 \\
\text { sessions delivered over } \\
10 \text { weeks. Each session } \\
\text { included } 100 \text { production } \\
\text { trials. }\end{array}$ & $6 / 7$ \\
\hline $\begin{array}{l}\text { Miccio and Ingrisano } \\
\quad(2000)\end{array}$ & $\begin{array}{l}\text { MBD across } \\
\text { behaviours }\end{array}$ & Minimal pairs & $n=1$ 'K' $(5 ; 3)$ & $\begin{array}{l}\text { Individual intervention } \\
\text { delivered by an SLT at } \\
\text { school. }\end{array}$ & $\begin{array}{l}4 \times 20-30-\text { min sessions } \\
\text { per week over } 29 \\
\text { weeks. }\end{array}$ & $4 / 7$ \\
\hline Monahan (1986) & Case study & Monahan Program & $n=4(5 ; 5-5 ; 8)$ & $\begin{array}{l}\text { Small group intervention } \\
\text { delivered by an SLT at } \\
\text { school. }\end{array}$ & $\begin{array}{l}2 \times 30 \text {-min sessions per } \\
\text { week for between } 10 \\
\text { and } 40 \text { sessions } \\
\text { delivered over } 2 \text { to } 8 \\
\text { months. }\end{array}$ & $5 / 7$ \\
\hline $\begin{array}{l}\text { Montgomery and } \\
\text { Bonderman (1989) }\end{array}$ & Case study & Cycles & $n=9(3 ; 1-4 ; 10)$ & $\begin{array}{l}\text { Group intervention } \\
\text { delivered by an SLT } \\
\text { and a paraprofessional } \\
\text { in a school. }\end{array}$ & $\begin{array}{l}3 \times 2 \text {-hour sessions per } \\
\text { week for a total of } \\
\text { between } 66 \text { and } 100 \\
\text { hours of intervention } \\
\text { (average } 90 \text { hours) } \\
\text { delivered over } 17 \text { weeks } \\
\text { of intervention within a } \\
7 \text {-month time period. } \\
\text { Intervention was } \\
\text { provided from referral } \\
\text { to discharge. }\end{array}$ & $5 / 7$ \\
\hline $\begin{array}{l}\text { Morrisette and Gierut } \\
\text { (2002) }\end{array}$ & $\begin{array}{l}\text { Staggered MBD } \\
\text { across } \\
\text { participants }\end{array}$ & $\begin{array}{l}\text { Generic phonological } \\
\text { approach, with } \\
\text { lexical properties } \\
\text { of targets varied }\end{array}$ & $n=8(3 ; 10-5 ; 4)$ & $\begin{array}{l}\text { Individual intervention } \\
\text { delivered by an SLT in } \\
\text { a university clinic. }\end{array}$ & $\begin{array}{l}3 \times 60 \text {-min sessions per } \\
\text { week for a total of } \\
\text { between } 5 \text { and } 19 \\
\text { sessions (average } 11 \text { ) } \\
\text { over } 2-8 \text { weeks } \\
\text { (average } 6 \text { ). }\end{array}$ & $5 / 7$ \\
\hline $\begin{array}{l}\text { Mota, Bagetti, Keske- } \\
\text { Soares and Pereira } \\
(2005)\end{array}$ & Case study & $\begin{array}{l}\text { Complexity: } \\
\text { Maximal } \\
\text { oppositions }\end{array}$ & $n=4(5 ; 3-7 ; 5)$ & $\begin{array}{l}\text { Delivered by an SLT in a } \\
\text { university clinic. }\end{array}$ & 15-25 sessions delivered. & $2 / 7$ \\
\hline
\end{tabular}




\begin{tabular}{|c|c|c|c|c|c|c|}
\hline Reference & Research design & $\begin{array}{l}\text { Intervention } \\
\text { approach }\end{array}$ & $\begin{array}{c}\text { Participant } \\
\text { number and age } \\
\text { (years;months) }\end{array}$ & $\begin{array}{c}\text { Summary of service } \\
\text { delivery }^{\mathrm{a}}\end{array}$ & $\begin{array}{c}\text { Summary of intervention } \\
\text { intensity }^{\mathrm{a}}\end{array}$ & $\begin{array}{c}\text { Reporting of } \\
\text { intervention } \\
\text { intensity }^{\mathbf{b}}\end{array}$ \\
\hline $\begin{array}{l}\text { Mota, Keske-Soares, } \\
\text { Bagetti, Ceron and } \\
\text { Filha (2007) }\end{array}$ & Non-RCT & $\begin{array}{l}\text { Cycles compared } \\
\text { with maximal } \\
\text { oppositions } \\
\text { compared with } \\
\text { ABAB withdrawal } \\
\text { and multiple } \\
\text { probes }\end{array}$ & $n=21(4 ; 0-7 ; 10)$ & Unclear. & Unclear. & $1 / 7$ \\
\hline $\begin{array}{l}\text { Nelson, Nygren, Walker } \\
\text { and Panoscha (2006) }\end{array}$ & Systematic review & $\begin{array}{l}\text { A range of speech } \\
\text { and language } \\
\text { interventions }\end{array}$ & $\begin{array}{l}25 \text { RCTs included in } \\
24 \text { publications }\end{array}$ & $\begin{array}{l}\text { Varied across included } \\
\text { studies. }\end{array}$ & $\begin{array}{l}\text { Varied across included } \\
\text { studies. }\end{array}$ & - \\
\hline $\begin{array}{l}\text { Olswang and Bain } \\
\text { (1985) }\end{array}$ & $\begin{array}{l}\text { Combined MBD } \\
\text { across behaviours } \\
\text { and ABA(B) } \\
\text { withdrawal } \\
\text { design }\end{array}$ & $\begin{array}{l}\text { Traditional } \\
\quad \text { articulation } \\
\text { intervention }\end{array}$ & $n=3(4 ; 0-4 ; 9)$ & $\begin{array}{l}\text { Individual intervention } \\
\text { delivered by an SLT } \\
\text { student in a university } \\
\text { clinic. }\end{array}$ & $\begin{array}{l}2-3 \times 50 \text {-min sessions per } \\
\text { week delivered over } 28- \\
38 \text { weeks. Intervention } \\
\text { was provided from } \\
\text { referral to discharge. }\end{array}$ & $4 / 7$ \\
\hline $\begin{array}{l}\text { Pagliarin, Brancalioni } \\
\text { and Keske-Soares } \\
\text { (2012) }\end{array}$ & $\begin{array}{l}\text { Between groups } \\
\text { design }\end{array}$ & $\begin{array}{l}\text { Multiple oppositions } \\
\text { compared with } \\
\text { ABAB withdrawal } \\
\text { and multiple } \\
\text { probes }\end{array}$ & $n=10(4 ; 8-7 ; 3)$ & $\begin{array}{l}\text { Delivered by an SLT in a } \\
\text { university clinic. }\end{array}$ & $\begin{array}{l}2 \times 45 \text {-min sessions per } \\
\text { week for } 15-30 \\
\text { sessions. }\end{array}$ & $4 / 7$ \\
\hline
\end{tabular}




\begin{tabular}{|c|c|c|c|c|c|c|}
\hline Reference & Research design & $\begin{array}{l}\text { Intervention } \\
\text { approach }\end{array}$ & $\begin{array}{c}\text { Participant } \\
\text { number and age } \\
\text { (years;months) }\end{array}$ & $\begin{array}{c}\text { Summary of service } \\
\text { delivery }^{\mathrm{a}}\end{array}$ & $\begin{array}{l}\text { Summary of intervention } \\
\text { intensity }\end{array}$ & $\begin{array}{l}\text { Reporting of } \\
\text { intervention } \\
\text { intensity }^{b}\end{array}$ \\
\hline $\begin{array}{l}\text { Pagliarin, Mota and } \\
\text { Keske-Soares (2009) }\end{array}$ & $\begin{array}{l}\text { Between groups } \\
\text { design }\end{array}$ & $\begin{array}{l}\text { Minimal pairs } \\
\text { compared with } \\
\text { multiple } \\
\text { oppositions } \\
\text { compared with } \\
\text { maximal } \\
\text { oppositions }\end{array}$ & $n=9(4 ; 2-6 ; 6)$ & $\begin{array}{l}\text { Delivered by an SLT in a } \\
\text { university clinic. }\end{array}$ & $\begin{array}{l}2 \times 45 \text {-min sessions per } \\
\text { week for between } 15 \\
\text { and } 25 \text { sessions. }\end{array}$ & $4 / 7$ \\
\hline $\begin{array}{l}\text { Pagliarin, Mota and } \\
\text { Keske-Soares (2011) }\end{array}$ & $\begin{array}{l}\text { Between groups } \\
\text { design }\end{array}$ & $\begin{array}{l}\text { Minimal pairs } \\
\text { compared with } \\
\text { multiple } \\
\text { oppositions } \\
\text { compared with } \\
\text { maximal } \\
\text { oppositions }\end{array}$ & $n=9(4 ; 2-6 ; 6)$ & $\begin{array}{l}\text { Delivered by an SLT in a } \\
\text { university clinic. }\end{array}$ & $\begin{array}{l}2 \times 45 \text {-min sessions per } \\
\text { week for between } 15 \\
\text { and } 25 \text { sessions. }\end{array}$ & $4 / 7$ \\
\hline $\begin{array}{l}\text { Palle, Berntsson, } \\
\text { Miniscalco and } \\
\text { Persson (2014) }\end{array}$ & $\begin{array}{l}\text { MBD across } \\
\text { behaviours }\end{array}$ & $\begin{array}{l}\text { Generic phonological } \\
\text { approach }\end{array}$ & $n=6(4 ; 1-5 ; 7)$ & $\begin{array}{l}\text { Individual intervention } \\
\text { delivered by an SLT in } \\
\text { a hospital clinic. }\end{array}$ & $\begin{array}{l}1 \times \text { session per week for } \\
\text { between } 6-18 \text { sessions } \\
\text { delivered over } 6-18 \\
\text { weeks. }\end{array}$ & $4 / 7$ \\
\hline $\begin{array}{l}\text { Pamplona, Ysunza and } \\
\text { Espinosa (1999) }\end{array}$ & $\mathrm{RCT}$ & $\begin{array}{l}\text { Traditional } \\
\text { articulation } \\
\text { compared with a } \\
\text { generic } \\
\text { phonological } \\
\text { approach }\end{array}$ & $n=29(3 ; 1-7 ; 1)$ & $\begin{array}{l}\text { Group intervention } \\
\text { delivered by an SLT in } \\
\text { a hospital clinic. }\end{array}$ & $\begin{array}{l}2 \times 60 \text {-min sessions per } \\
\text { week, delivered over } 6- \\
46 \text { months. Intervention } \\
\text { was provided from } \\
\text { referral to discharge. }\end{array}$ & $4 / 7$ \\
\hline $\begin{array}{l}\text { Pamplona, Ysunza and } \\
\text { Morales (2014) }\end{array}$ & RCT & $\begin{array}{l}\text { Whole language with } \\
\text { generic } \\
\text { phonological } \\
\text { principles }\end{array}$ & $n=90(3-6 ; 8)$ & $\begin{array}{l}\text { Group intervention } \\
\text { delivered by an SLT in } \\
\text { a hospital clinic. }\end{array}$ & $\begin{array}{l}\text { 4-hours of intervention per } \\
\text { day }(5 \times \text { weekly) for } 4 \\
\text { weeks. }\end{array}$ & $4 / 7$ \\
\hline $\begin{array}{l}\text { Pamplona, Ysunza and } \\
\text { Ramırez (2004) }\end{array}$ & RCT & $\begin{array}{l}\text { Generic phonological } \\
\text { approach } \\
\text { compared with } \\
\text { whole language }\end{array}$ & $n=30(3 ; 0-7 ; 2)$ & $\begin{array}{l}\text { Group intervention } \\
\text { delivered by an SLT in } \\
\text { a hospital clinic. }\end{array}$ & $\begin{array}{l}2 \times 60 \text {-min sessions per } \\
\text { week, delivered over } 4 \\
\text { to } 27 \text { months. } \\
\text { Intervention was } \\
\text { provided from referral } \\
\text { to discharge. }\end{array}$ & $4 / 7$ \\
\hline
\end{tabular}




\begin{tabular}{|c|c|c|c|c|c|c|}
\hline Reference & Research design & $\begin{array}{l}\text { Intervention } \\
\text { approach }\end{array}$ & $\begin{array}{c}\text { Participant } \\
\text { number and age } \\
\text { (years;months) }\end{array}$ & $\begin{array}{c}\text { Summary of service } \\
\text { delivery }^{\mathrm{a}}\end{array}$ & $\begin{array}{c}\text { Summary of intervention } \\
\text { intensity }\end{array}$ & $\begin{array}{c}\text { Reporting of } \\
\text { intervention } \\
\text { intensity }^{\mathbf{b}}\end{array}$ \\
\hline $\begin{array}{l}\text { Pascoe, Stackhouse and } \\
\text { Wells (2005) }\end{array}$ & Case study & $\begin{array}{l}\text { Psycholinguistic } \\
\text { approach }\end{array}$ & $n=1$ 'Katy’ $(6 ; 5)$ & $\begin{array}{l}\text { Individual intervention } \\
\text { delivered by an SLT at } \\
\text { school. }\end{array}$ & $\begin{array}{l}2 \times 60 \text {-min sessions per } \\
\text { week delivered over } 3 \times \\
\text { blocks of } 10 \text { sessions } \\
\text { each. In total, } 30 \\
\text { sessions of intervention } \\
\text { delivered over } 7 \\
\text { months, with a } 7 \text {-month } \\
\text { follow-up. }\end{array}$ & $5 / 7$ \\
\hline $\begin{array}{l}\text { Penney, Fee and Dowdle } \\
\text { (1994) }\end{array}$ & Case study & Vowel intervention & $n=1$ 'CG' $(4 ; 11)$ & $\begin{array}{l}\text { Individual intervention } \\
\text { delivered by an SLT in } \\
\text { a university clinic. }\end{array}$ & - & $1 / 7$ \\
\hline $\begin{array}{l}\text { Pieretti, Kaul, Zarchy } \\
\text { and O’Hanlon (2015) }\end{array}$ & $\begin{array}{l}\text { Multiple baseline } \\
\text { ABCA design }\end{array}$ & $\begin{array}{l}\text { Traditional } \\
\text { articulation } \\
\text { therapy compared } \\
\text { with a multimodal } \\
\text { phonological } \\
\text { approach }\end{array}$ & $n=2(4 ; 1-4 ; 3)$ & $\begin{array}{l}\text { Individual intervention } \\
\text { delivered by an SLT in } \\
\text { a preschool. }\end{array}$ & $\begin{array}{l}\text { 30-min sessions delivered } \\
\text { 'biweekly' (p. 135, } \\
\text { coded as unclear) for } 20 \\
\text { sessions delivered over } \\
20 \text { weeks. }\end{array}$ & $4 / 7$ \\
\hline Pollock (1983) & Case study & $\begin{array}{l}\text { Traditional } \\
\text { articulation } \\
\text { intervention }\end{array}$ & $n=1$ 'Mike' $(3 ; 5)$ & $\begin{array}{l}\text { Individual and group } \\
\text { intervention delivered } \\
\text { by an SLT. }\end{array}$ & $\begin{array}{l}\text { Reports on intervention } \\
\text { delivered over a period } \\
\text { of } 2 \text { years and } 5 \text { months. }\end{array}$ & $2 / 7$ \\
\hline Powell (1991) & Case study & Minimal pairs & $n=1$ 'JA' $(5 ; 8)$ & $\begin{array}{l}\text { Individual intervention } \\
\text { delivered by an SLT in } \\
\text { a university clinic. }\end{array}$ & $\begin{array}{l}15 \times 30 \text {-min sessions } \\
\text { delivered, each } \\
\text { comprising a production } \\
\text { dose of } 100 \text { trials. }\end{array}$ & $4 / 7$ \\
\hline Powell (1993) & $\begin{array}{l}\text { MBD across } \\
\text { behaviours }\end{array}$ & Minimal pairs & $n=6(4 ; 11-5 ; 6)$ & $\begin{array}{l}\text { Individual intervention } \\
\text { delivered by an SLT in } \\
\text { a university clinic. }\end{array}$ & $\begin{array}{l}3 \times 30 \text {-min sessions per } \\
\text { week for a total of } 13- \\
38 \text { sessions. Each } \\
\text { session comprised a } \\
\text { production dose of } 100 \\
\text { trials. }\end{array}$ & $5 / 7$ \\
\hline
\end{tabular}




\begin{tabular}{|c|c|c|c|c|c|c|}
\hline Reference & Research design & $\begin{array}{l}\text { Intervention } \\
\text { approach }\end{array}$ & $\begin{array}{c}\text { Participant } \\
\text { number and age } \\
\text { (years;months) }\end{array}$ & $\begin{array}{c}\text { Summary of service } \\
\text { delivery }^{\mathrm{a}}\end{array}$ & $\begin{array}{l}\text { Summary of intervention } \\
\text { intensity }\end{array}$ & $\begin{array}{l}\text { Reporting of } \\
\text { intervention } \\
\text { intensity }^{b} \\
\end{array}$ \\
\hline Powell and Elbert (1984) & $\begin{array}{l}\text { MBD across } \\
\text { participants }\end{array}$ & Minimal pairs & $n=6(4 ; 4-6 ; 3)$ & $\begin{array}{l}\text { Individual intervention } \\
\text { delivered by a } \\
\text { researcher in a } \\
\text { university clinic. }\end{array}$ & $\begin{array}{l}\text { 30-min sessions each } \\
\text { comprising a production } \\
\text { dose of } 100 \text { trials. } \\
\text { Excluding baseline } \\
\text { sessions, the total } \\
\text { duration of intervention } \\
\text { was between } 1 \text { and } 4 \\
\text { months (total study } \\
\text { duration of } 5-9 \\
\text { months). }\end{array}$ & $4 / 7$ \\
\hline $\begin{array}{r}\text { Powell, Elbert and } \\
\text { Dinnsen (1991) }\end{array}$ & $\begin{array}{l}\text { MBD across } \\
\text { behaviours }\end{array}$ & Minimal pairs & $n=6(4 ; 11-5 ; 6)$ & $\begin{array}{r}\text { Individual intervention } \\
\text { delivered by an SLT. }\end{array}$ & $\begin{array}{l}3 \times 30 \text {-min sessions per } \\
\text { week, with each session } \\
\text { comprising a production } \\
\text { dose of } 100 \text { trials. In } \\
\text { total, each participant } \\
\text { received between } 14 \\
\text { and } 39 \text { intervention } \\
\text { sessions. }\end{array}$ & $5 / 7$ \\
\hline $\begin{array}{l}\text { Powell, Elbert, Miccio, } \\
\text { Strike-roussos and } \\
\text { Brasseur (1998) }\end{array}$ & $\begin{array}{l}\text { MBD across } \\
\text { participants }\end{array}$ & $\begin{array}{l}\text { Minimal pairs } \\
\text { (conceptual- } \\
\text { listening tasks } \\
\text { only) compared } \\
\text { with traditional } \\
\text { articulation } \\
\text { intervention }\end{array}$ & $n=18(3 ; 6-6 ; 10)$ & $\begin{array}{l}\text { Individual intervention } \\
\text { delivered by an SLT in } \\
\text { a university clinic. }\end{array}$ & $\begin{array}{l}\text { 40-min sessions delivered } \\
\text { 'several times per week' } \\
\text { (p. 152) [note: coded as } \\
\text { unclear]. Each session } \\
\text { comprised a production } \\
\text { dose of } 100 \text {. In total, } \\
\text { children receiving the } \\
\text { phonological } \\
\text { intervention received } 20 \\
\text { sessions of intervention } \\
\text { delivered over } 9-12 \\
\text { weeks. }\end{array}$ & $6 / 7$ \\
\hline
\end{tabular}




\begin{tabular}{|c|c|c|c|c|c|c|}
\hline Reference & Research design & $\begin{array}{l}\text { Intervention } \\
\text { approach }\end{array}$ & $\begin{array}{c}\text { Participant } \\
\text { number and age } \\
\text { (years;months) }\end{array}$ & $\begin{array}{c}\text { Summary of service } \\
\text { delivery }^{\mathrm{a}}\end{array}$ & $\begin{array}{l}\text { Summary of intervention } \\
\text { intensity }\end{array}$ & $\begin{array}{c}\text { Reporting of } \\
\text { intervention } \\
\text { intensity }^{b}\end{array}$ \\
\hline Ray (2002) & Case study & Minimal pairs & $n=1$ 'MC' (5) & $\begin{array}{l}\text { Individual intervention } \\
\text { delivered by an SLT at a } \\
\text { university clinic and at } \\
\text { home. }\end{array}$ & $\begin{array}{l}3 \times 45-60 \text { min sessions per } \\
\text { week for a total of } 40 \\
\text { sessions delivered over } \\
20 \text { weeks ( } 5 \text { months). In } \\
\text { each session, a } \\
\text { perceptual dose of } 20 \\
\text { was provided; } \\
\text { production activities } \\
\text { were also included in } \\
\text { the intervention, but no } \\
\text { details on dose were } \\
\text { provided. }\end{array}$ & $5 / 7$ \\
\hline $\begin{array}{l}\text { Robb, Bleile and Yee } \\
\text { (1999) }\end{array}$ & Case study & Minimal pairs & $n=1$ 'Jenny' $(4 ; 0)$ & $\begin{array}{l}\text { Individual intervention } \\
\text { delivered by the } \\
\text { researcher in a } \\
\text { university clinic. }\end{array}$ & $\begin{array}{l}2 \times 45 \text {-min sessions per } \\
\text { week for a total of } 20 \\
\text { sessions delivered over } \\
10 \text { weeks. }\end{array}$ & $5 / 7$ \\
\hline $\begin{array}{l}\text { Rudolph and Wendt } \\
\text { (2014) }\end{array}$ & $\begin{array}{l}\text { MBD across } \\
\text { behaviours }\end{array}$ & Cycles & $n=3(4 ; 3-5 ; 3)$ & $\begin{array}{l}\text { Individual intervention } \\
\text { delivered by an SLT in } \\
\text { a university clinic. }\end{array}$ & $\begin{array}{l}3 \times 60 \text {-min sessions per } \\
\text { week for } 18 \text { sessions. } \\
\text { Intervention was } \\
\text { delivered over two } \\
\text { blocks, each } 3 \text {-weeks ( } 9 \\
\text { sessions) in duration, } \\
\text { separated by a 1-week } \\
\text { break. }\end{array}$ & $5 / 7$ \\
\hline $\begin{array}{l}\text { Ruscello, Cartwright, } \\
\text { Haines and Shuster } \\
\text { (1993) }\end{array}$ & $\mathrm{RCT}$ & Minimal pairs & $n=12(4 ; 1-5 ; 8)$ & $\begin{array}{l}\text { Group I: Individual } \\
\text { intervention delivered } \\
\text { by an SLT in a } \\
\text { university clinic. } \\
\text { Group II: Individual } \\
\text { intervention delivered } \\
\text { by an SLT and a parent } \\
\text { in a university clinic. }\end{array}$ & $\begin{array}{l}\text { Both groups: } 2 \times 60 \text {-min } \\
\text { sessions per week for a } \\
\text { total of } 16 \text { sessions } \\
\text { delivered over } 8 \text { weeks. }\end{array}$ & $5 / 7$ \\
\hline
\end{tabular}




\begin{tabular}{|c|c|c|c|c|c|c|}
\hline Reference & Research design & $\begin{array}{l}\text { Intervention } \\
\text { approach }\end{array}$ & $\begin{array}{c}\text { Participant } \\
\text { number and age } \\
\text { (years;months) }\end{array}$ & $\begin{array}{c}\text { Summary of service } \\
\text { delivery }^{\mathrm{a}}\end{array}$ & $\begin{array}{l}\text { Summary of intervention } \\
\text { intensity }\end{array}$ & $\begin{array}{l}\text { Reporting of } \\
\text { intervention } \\
\text { intensity }^{b}\end{array}$ \\
\hline Rvachew (1994) & RCT & $\begin{array}{l}\text { Speech perception } \\
\text { training (using } \\
\text { SAILS) plus } \\
\text { traditional } \\
\text { articulation } \\
\text { intervention }\end{array}$ & $n=27(3 ; 6-5 ; 6)$ & $\begin{array}{l}\text { Individual intervention } \\
\text { delivered by an SLT in } \\
\text { a hospital clinic. }\end{array}$ & $\begin{array}{l}1 \times 45 \text {-min session per } \\
\text { week for a total of } 6 \\
\text { intervention sessions } \\
\text { delivered over } 6 \text { weeks. } \\
\text { Each session comprised } \\
60 \text { perception trials and } \\
60 \text { production trials. }\end{array}$ & $6 / 7$ \\
\hline $\begin{array}{l}\text { Rvachew and Bernhardt } \\
\text { (2010) }\end{array}$ & $\begin{array}{l}\text { Analysis of a } \\
\text { subgroup of } \\
\text { children who } \\
\text { participated in an } \\
\text { RCT (Rvachew } \\
\text { and Nowak, } \\
\text { 2001) }\end{array}$ & $\begin{array}{l}\text { Complexity approach } \\
\text { targeting later } \\
\text { developing/least } \\
\text { knowledge } \\
\text { phonemes } \\
\text { compared with a } \\
\text { developmental } \\
\text { goal approach }\end{array}$ & $n=6(3 ; 5-4 ; 4)$ & $\begin{array}{l}\text { Individual intervention } \\
\text { delivered by an SLT in } \\
\text { a hospital clinic. }\end{array}$ & $\begin{array}{l}1 \times 30-40 \text { min session per } \\
\text { week for a total of } 12 \\
\text { intervention sessions } \\
\text { delivered over } 12-14 \\
\text { weeks. }\end{array}$ & $5 / 7$ \\
\hline $\begin{array}{l}\text { Rvachew and Brosseau- } \\
\text { Lapré (2015) }\end{array}$ & $\mathrm{RCT}$ & $\begin{array}{l}\text { Different } \\
\text { combinations of } \\
\text { input- and output- } \\
\text { oriented } \\
\text { interventions with } \\
\text { phonological } \\
\text { awareness training }\end{array}$ & $\begin{array}{l}n=65\left(\mathrm{M}_{\text {age }} \text { of }\right. \\
\quad \text { treatment groups } \\
=52.25-54.08 \\
\quad \text { months })\end{array}$ & $\begin{array}{l}\text { Intervention delivered over } \\
\text { two blocks. In the first } \\
\text { block, participants } \\
\text { received individual } \\
\text { intervention delivered } \\
\text { by an SLT student in a } \\
\text { hospital clinic. In the } \\
\text { second block, } \\
\text { participants received } \\
\text { group intervention from } \\
\text { an SLT student in a } \\
\text { hospital clinic while } \\
\text { parents attended a group } \\
\text { training session. }\end{array}$ & $\begin{array}{l}\text { Block 1: } 1 \times 45 \text {-min } \\
\text { session per week for a } \\
\text { total of up to } 6 \text { sessions } \\
\text { (group averages }=5.15- \\
\text { 5.44) over } 6 \text { weeks. } \\
\text { Block 2: (group } \\
\text { averages = } 4.77-5.13 \text { ) } \\
\text { over } 6 \text { weeks. In total, } \\
\text { participants in each } \\
\text { group took an average } \\
\text { of } 13.33-14.92 \text { weeks to } \\
\text { complete the } \\
\text { intervention program. }\end{array}$ & $5 / 7$ \\
\hline
\end{tabular}




\begin{tabular}{|c|c|c|c|c|c|c|}
\hline Reference & Research design & $\begin{array}{l}\text { Intervention } \\
\text { approach }\end{array}$ & $\begin{array}{c}\text { Participant } \\
\text { number and age } \\
\text { (years;months) }\end{array}$ & $\begin{array}{c}\text { Summary of service } \\
\text { delivery }^{\mathrm{a}}\end{array}$ & $\begin{array}{l}\text { Summary of intervention } \\
\text { intensity }\end{array}$ & $\begin{array}{l}\text { Reporting of } \\
\text { intervention } \\
\text { intensity }^{b} \\
\end{array}$ \\
\hline $\begin{array}{l}\text { Rvachew and Nowak } \\
\quad \text { (2001) }\end{array}$ & RCT & $\begin{array}{l}\text { Complexity approach } \\
\text { targeting later } \\
\text { developing/least } \\
\text { knowledge } \\
\text { phonemes } \\
\text { compared with a } \\
\text { developmental } \\
\text { goal approach }\end{array}$ & $\begin{array}{c}n=48\left(\text { Group I } \mathrm{M}_{\text {age }}\right. \\
=51.46 \text { months } \\
\quad \text { Group II M }_{\text {age }}= \\
49.63 \text { months })\end{array}$ & $\begin{array}{l}\text { Individual intervention } \\
\text { delivered by an SLT in } \\
\text { a hospital clinic. }\end{array}$ & $\begin{array}{l}1 \times 30-40 \text {-min session per } \\
\text { week for a total of } 12 \\
\text { intervention sessions } \\
\text { delivered over } 12-14 \\
\text { weeks. }\end{array}$ & $5 / 7$ \\
\hline $\begin{array}{l}\text { Rvachew, Nowak and } \\
\text { Cloutier (2004) }\end{array}$ & RCT & $\begin{array}{l}\text { Speech perception } \\
\text { training (SAILS) } \\
\text { alongside regular } \\
\text { speech therapy } \\
\text { (using a range of } \\
\text { approaches) }\end{array}$ & $n=34(3 ; 5-4 ; 11)$ & $\begin{array}{l}\text { Individual intervention } \\
\text { delivered by an SLT } \\
\text { student or parent in a } \\
\text { hospital (delivered in } \\
\text { addition to regular } \\
\text { speech and language } \\
\text { therapy services). }\end{array}$ & $\begin{array}{l}1 \times 15 \text {-min session per } \\
\text { week for an average of } \\
12 \text { sessions over } 4.7 \\
\text { months (delivered in } \\
\text { addition to regular } \\
\text { speech and language } \\
\text { therapy services). Each } \\
\text { session provided a } \\
\text { minimum perception } \\
\text { dose of } 120 \text { trials. }\end{array}$ & $6 / 7$ \\
\hline \multirow[t]{2}{*}{$\begin{array}{l}\text { Rvachew, Rafaat and } \\
\text { Martin (1999) }\end{array}$} & \multirow[t]{2}{*}{$\begin{array}{l}\text { Within subjects } \\
\text { design }\end{array}$} & $\begin{array}{l}\text { Study I: Modified } \\
\text { cycles approach }\end{array}$ & $\begin{array}{l}\text { Study I: } n=10 \\
\quad(4 ; 2-4 ; 11)\end{array}$ & $\begin{array}{l}\text { Study I: Group } \\
\text { intervention delivered } \\
\text { by an SLT in a hospital } \\
\text { clinic. }\end{array}$ & $\begin{array}{l}\text { Study I: } 1 \times 45-60 \text {-min } \\
\text { sessions per week for a } \\
\text { total of } 9 \text { sessions } \\
\text { delivered over } 9 \text { weeks. }\end{array}$ & $5 / 7$ \\
\hline & & $\begin{array}{l}\text { Study II: Speech } \\
\text { perception } \\
\text { training (SAILS) } \\
\text { plus modified } \\
\text { cycles and } \\
\text { stimulability } \\
\text { training }\end{array}$ & $\begin{array}{l}\text { Study II: } n=13 \\
\quad(3 ; 9-4 ; 11)\end{array}$ & $\begin{array}{l}\text { Study II: Individual } \\
\text { intervention delivered } \\
\text { by an SLT and a speech } \\
\text { aide in a hospital clinic, } \\
\text { followed by group } \\
\text { intervention delivered } \\
\text { by an SLT in a hospital } \\
\text { clinic. }\end{array}$ & $\begin{array}{l}\text { Study II: } 1 \times 20 \text {-min } \\
\text { session per week for a } \\
\text { total of } 3 \text { sessions } \\
\text { delivered over } 3 \text { weeks, } \\
\text { followed by } 1 \times 45-60- \\
\text { min session per week } \\
\text { for } 6 \text { weeks. In total, } 9 \\
\text { sessions of intervention } \\
\text { delivered over } 9 \text { weeks. }\end{array}$ & $5 / 7$ \\
\hline
\end{tabular}




\begin{tabular}{|c|c|c|c|c|c|c|}
\hline Reference & Research design & $\begin{array}{l}\text { Intervention } \\
\text { approach }\end{array}$ & $\begin{array}{c}\text { Participant } \\
\text { number and age } \\
\text { (years;months) }\end{array}$ & $\begin{array}{c}\text { Summary of service } \\
\text { delivery }^{\mathrm{a}}\end{array}$ & $\begin{array}{l}\text { Summary of intervention } \\
\text { intensity }\end{array}$ & $\begin{array}{l}\text { Reporting of } \\
\text { intervention } \\
\text { intensity }^{b}\end{array}$ \\
\hline $\begin{array}{l}\text { Saben and Costello } \\
\text { Ingham (1991) }\end{array}$ & Case study & Minimal pairs & $n=2(3 ; 9-4 ; 4)$ & $\begin{array}{l}\text { Individual intervention } \\
\text { delivered by an SLT. }\end{array}$ & $\begin{array}{l}\text { Participant } 1 \text { (age } 4 ; 4) \\
\text { received } 67 \text { sessions of } \\
\text { intervention delivered } \\
\text { over } 9.5 \text { months. } \\
\text { Participant } 2 \text { (age } 3 ; 9) \\
\text { received } 32 \text { sessions } \\
\text { delivered over } 4.5 \\
\text { months. }\end{array}$ & $3 / 7$ \\
\hline $\begin{array}{l}\text { Seeff-Gabriel, Chiat and } \\
\text { Pring (2012) }\end{array}$ & Case study & $\begin{array}{l}\text { Generic phonological } \\
\text { approach } \\
\text { combined with } \\
\text { elements of } \\
\text { traditional } \\
\text { articulation and } \\
\text { morphosyntax } \\
\text { intervention }\end{array}$ & $n=1$ 'B' $(5 ; 1)$ & $\begin{array}{l}\text { Individual intervention } \\
\text { delivered by an SLT in } \\
\text { a university clinic. }\end{array}$ & $\begin{array}{l}1 \times 30 \text {-min session per } \\
\text { week for a total of } 20 \\
\text { sessions delivered over } \\
20 \text { weeks. }\end{array}$ & $5 / 7$ \\
\hline Shea and Tyler (2001) & $\begin{array}{l}\text { MBD across } \\
\text { participants }\end{array}$ & $\begin{array}{l}\text { Prosodic intervention } \\
\text { targeting stress } \\
\text { patterns }\end{array}$ & $n=2(3 ; 1-3 ; 7)$ & $\begin{array}{l}\text { Individual intervention } \\
\text { delivered by an SLT. }\end{array}$ & $\begin{array}{l}3 \times 45 \text {-min sessions per } \\
\text { week delivered over } 4 \\
\text { months. Participant } 1 \\
\text { attended } 28 \text { sessions (of } \\
\text { which } 16 \text { were } \\
\text { intervention) and } \\
\text { participant } 2 \text { attended } \\
22 \text { sessions (12 } \\
\text { intervention). }\end{array}$ & $5 / 7$ \\
\hline $\begin{array}{l}\text { Shiller, Rvachew and } \\
\text { Brosseau-Lapré } \\
(2010)\end{array}$ & Case study & $\begin{array}{l}\text { Speech perception } \\
\quad \text { (SAILS), focused } \\
\text { stimulation, and } \\
\text { minimal pairs }\end{array}$ & $n=1(4 ; 8)$ & $\begin{array}{l}\text { Individual intervention } \\
\text { delivered by an SLT in } \\
\text { a university clinic. }\end{array}$ & $\begin{array}{l}6 \text { sessions of intervention } \\
\text { delivered over } 6 \text { weeks. }\end{array}$ & $3 / 7$ \\
\hline $\begin{array}{l}\text { Shoaf, Iyer and Bothe } \\
\text { (2009) }\end{array}$ & $\mathrm{ABAB}$ design & $\begin{array}{l}\text { Nonlinear } \\
\text { phonological } \\
\text { intervention }\end{array}$ & $n=1(6 ; 4)$ & $\begin{array}{l}\text { Individual and group } \\
\text { intervention delivered } \\
\text { by an SLT in a school. }\end{array}$ & $\begin{array}{l}4 \times 30 \text {-min sessions per } \\
\text { week for a total of } 18 \\
\text { sessions delivered over } \\
2 \text { months. }\end{array}$ & $5 / 7$ \\
\hline
\end{tabular}




\begin{tabular}{|c|c|c|c|c|c|c|}
\hline Reference & Research design & $\begin{array}{l}\text { Intervention } \\
\text { approach }\end{array}$ & $\begin{array}{c}\text { Participant } \\
\text { number and age } \\
\text { (years;months) }\end{array}$ & $\begin{array}{c}\text { Summary of service } \\
\text { delivery }^{\mathrm{a}}\end{array}$ & $\begin{array}{c}\text { Summary of intervention } \\
\text { intensity }\end{array}$ & $\begin{array}{c}\text { Reporting of } \\
\text { intervention } \\
\text { intensity }\end{array}$ \\
\hline $\begin{array}{l}\text { Shriberg and } \\
\quad \text { Kwiatkowski (1982) }\end{array}$ & $\begin{array}{l}\text { Within subjects } \\
\text { design }\end{array}$ & $\begin{array}{l}\text { Generic phonological } \\
\text { approach }\end{array}$ & $n=31(3 ; 10-9 ; 0)$ & $\begin{array}{l}\text { Group intervention } \\
\text { delivered by an SLT } \\
\text { student in a university } \\
\text { clinic. }\end{array}$ & $\begin{array}{l}4 \times \text { approx. 2-hour } \\
\text { sessions per week } \\
\text { delivered over 5-6 } \\
\text { weeks. Participants in } \\
\text { Group A received a } \\
\text { total of } 19 \text { sessions; } \\
\text { total number of sessions } \\
\text { was not reported for } \\
\text { participants in Groups B } \\
\text { and C. }\end{array}$ & $5 / 7$ \\
\hline $\begin{array}{l}\text { Shriberg and } \\
\text { Kwiatkowski (1987) }\end{array}$ & $\begin{array}{l}\text { Retrospective } \\
\text { within subjects } \\
\text { design }\end{array}$ & $\begin{array}{l}\text { A range of generic } \\
\text { phonological } \\
\text { approaches }\end{array}$ & $n=73(2 ; 9-9 ; 6)$ & $\begin{array}{l}\text { Individual intervention } \\
\text { delivered by an SLT } \\
\text { student in a university } \\
\text { clinic. }\end{array}$ & $\begin{array}{l}\text { A total of } 8-19 \text { sessions } \\
\quad \text { (average }=14) \text { delivered } \\
\text { over a university } \\
\text { semester. }\end{array}$ & $3 / 7$ \\
\hline $\begin{array}{l}\text { Shriberg and } \\
\quad \text { Kwiatkowski (1990) }\end{array}$ & Case study & $\begin{array}{l}\text { Self-monitoring } \\
\text { within a generic } \\
\text { phonological } \\
\text { approach }\end{array}$ & $n=8(3 ; 8-5 ; 7)$ & $\begin{array}{l}\text { Individual intervention } \\
\text { delivered by an SLT } \\
\text { student in a university } \\
\text { clinic. }\end{array}$ & $\begin{array}{l}2 \times 50 \text {-min sessions per } \\
\text { week delivered over a } \\
\text { university semester. } \\
\text { Each child received } \\
\text { treatment for two } \\
\text { targets, which required } \\
\text { between } 6 \text { and } 21 \\
\text { sessions to reach } \\
\text { termination criteria. }\end{array}$ & $5 / 7$ \\
\hline $\begin{array}{l}\text { Shriberg, Kwiatkowski } \\
\text { and Snyder (1989) }\end{array}$ & $\begin{array}{l}\text { Within subjects } \\
\text { design }\end{array}$ & $\begin{array}{l}\text { Generic phonological } \\
\text { approach }\end{array}$ & $n=18(3 ; 6-8 ; 9)$ & $\begin{array}{l}\text { Individual intervention } \\
\text { delivered by an SLT in } \\
\text { a university clinic. }\end{array}$ & $\begin{array}{l}\text { 60-min sessions delivered } \\
\text { over } 1-2 \text { weeks. }\end{array}$ & $3 / 7$ \\
\hline
\end{tabular}




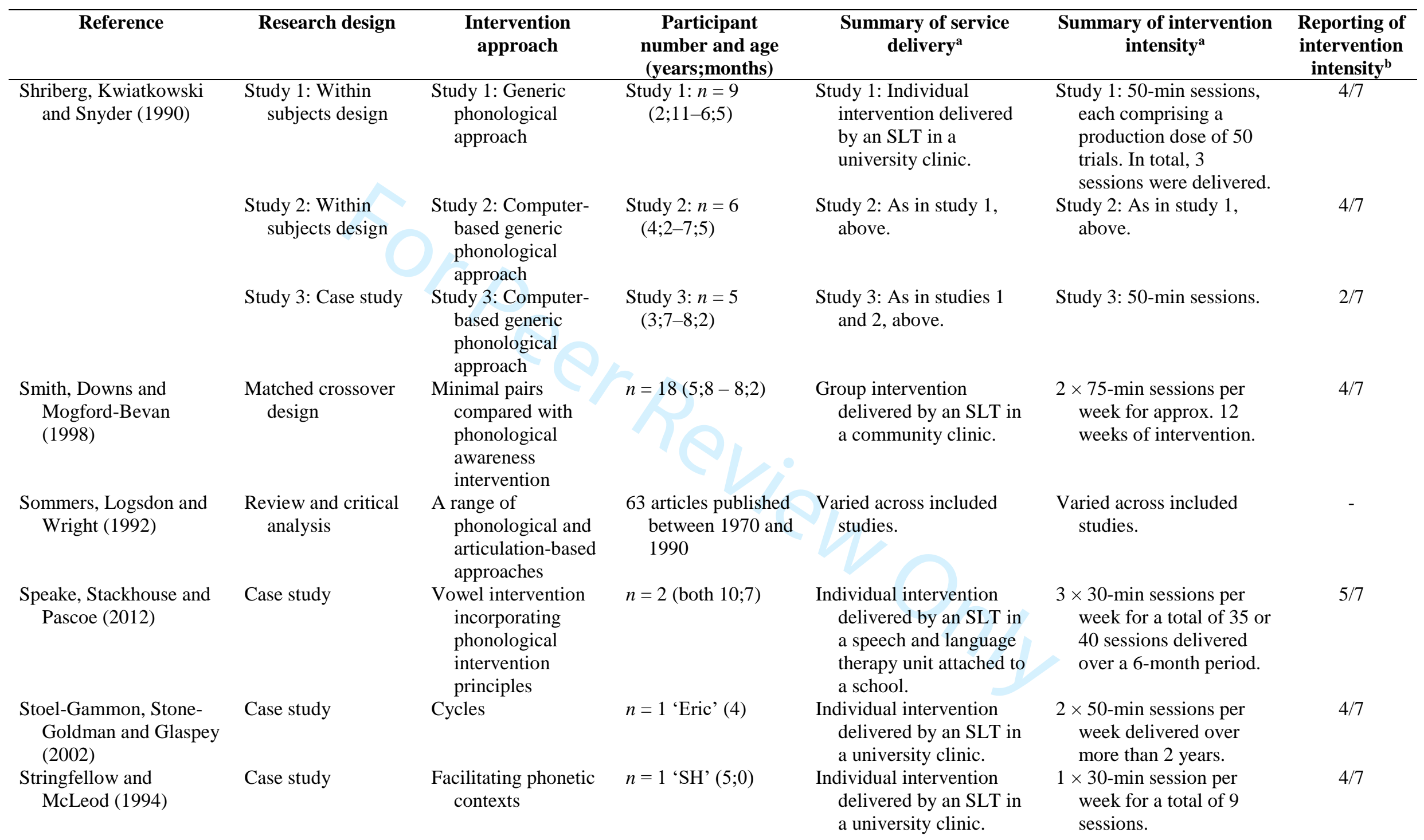




\begin{tabular}{|c|c|c|c|c|c|c|}
\hline Reference & Research design & $\begin{array}{c}\text { Intervention } \\
\text { approach }\end{array}$ & $\begin{array}{c}\text { Participant } \\
\text { number and age } \\
\text { (years;months) }\end{array}$ & $\begin{array}{c}\text { Summary of service } \\
\text { delivery }^{\mathrm{a}}\end{array}$ & $\begin{array}{c}\text { Summary of intervention } \\
\text { intensity }^{\mathrm{a}}\end{array}$ & $\begin{array}{c}\text { Reporting of } \\
\text { intervention } \\
\text { intensity }^{\mathbf{b}}\end{array}$ \\
\hline $\begin{array}{l}\text { Sugden, Baker, Munro } \\
\text { and Williams (2016) }\end{array}$ & $\begin{array}{l}\text { Systematic search } \\
\text { and review }\end{array}$ & $\begin{array}{l}\text { A range of } \\
\text { phonological and } \\
\text { articulation-based } \\
\text { approaches }\end{array}$ & $\begin{array}{l}61 \text { papers published } \\
\text { between } 1979 \text { and } \\
2013 \text { that } \\
\text { included parent } \\
\text { involvement or } \\
\text { home practice. }\end{array}$ & $\begin{array}{l}\text { Varied across included } \\
\text { studies. }\end{array}$ & $\begin{array}{l}\text { Varied across included } \\
\text { studies. }\end{array}$ & - \\
\hline Topbaş and Ünal (2010) & $\begin{array}{l}\text { ATD with staggered } \\
\text { MBD across } \\
\text { participants }\end{array}$ & $\begin{array}{l}\text { Complexity } \\
\text { approach: } \\
\text { Maximal } \\
\text { oppositions } \\
\text { compared with } \\
\text { minimal pairs }\end{array}$ & $n=2($ twins, $6 ; 0)$ & $\begin{array}{l}\text { Individual intervention } \\
\text { delivered by an SLT in } \\
\text { a university clinic. }\end{array}$ & $\begin{array}{l}3 \times 60 \text {-min sessions per } \\
\text { week for a total of } 10 \\
\text { sessions delivered over } \\
4 \text { weeks. }\end{array}$ & $5 / 7$ \\
\hline Tyler (1995) & Case study & Minimal pairs & $n=6(3 ; 11-5 ; 11)$ & $\begin{array}{l}\text { Individual intervention } \\
\text { delivered by an SLT in } \\
\text { a university clinic. }\end{array}$ & $\begin{array}{l}2 \times 45 \text {-min sessions per } \\
\text { week for between } 6 \text { and } \\
31 \text { sessions. }\end{array}$ & $4 / 7$ \\
\hline $\begin{array}{l}\text { Tyler, Edwards and } \\
\text { Saxman (1987) }\end{array}$ & $\mathrm{AB}$ design & $\begin{array}{l}\text { Minimal pairs } \\
\text { compared with } \\
\text { modified cycles }\end{array}$ & $n=4(3 ; 1-5 ; 1)$ & $\begin{array}{l}\text { Individual intervention } \\
\text { delivered by an SLT in } \\
\text { a university clinic. }\end{array}$ & $\begin{array}{l}2 \times 60 \text {-min sessions per } \\
\text { week for a total of } 12 \text { to } \\
25 \text { sessions delivered } \\
\text { over approx. } 8-10 \\
\text { weeks. Each session } \\
\text { comprised a perceptual } \\
\text { dose of } 50 \text { trials; } \\
\text { production dose } \\
\text { reported but unclear. }\end{array}$ & $6 / 7$ \\
\hline $\begin{array}{l}\text { Tyler, Edwards and } \\
\text { Saxman (1990) }\end{array}$ & $\begin{array}{l}\text { Case studies with a } \\
\text { control case }\end{array}$ & Minimal pairs & $n=4(4 ; 10-5 ; 3)$ & $\begin{array}{l}\text { Individual intervention } \\
\text { delivered by an SLT } \\
\text { student in a university } \\
\text { clinic. }\end{array}$ & $\begin{array}{l}2 \times 45 \text {-min sessions per } \\
\text { week for a total of } 16 \\
\text { sessions delivered over } \\
8 \text { weeks. }\end{array}$ & $5 / 7$ \\
\hline $\begin{array}{l}\text { Tyler and Figurski } \\
\text { (1994) }\end{array}$ & $\begin{array}{l}\text { Combined ABAB } \\
\text { with MPD }\end{array}$ & $\begin{array}{l}\text { Generic phonological } \\
\text { approach }\end{array}$ & $n=2(2 ; 8-2 ; 10)$ & $\begin{array}{l}\text { Individual intervention } \\
\text { delivered by an SLT in } \\
\text { a university clinic. }\end{array}$ & $\begin{array}{l}2 \times 9 \text {-week blocks of } \\
\text { intervention separated } \\
\text { by a } 5 \text { week withdrawal } \\
\text { period. }\end{array}$ & $2 / 7$ \\
\hline $\begin{array}{l}\text { Tyler, Figurski and } \\
\text { Langsdale (1993) }\end{array}$ & $\begin{array}{l}\text { MBD across } \\
\text { participants }\end{array}$ & Minimal pairs & $n=7(3 ; 10-5 ; 6)$ & $\begin{array}{l}\text { Individual intervention } \\
\text { delivered by the } \\
\text { researchers in a } \\
\text { university clinic. }\end{array}$ & $\begin{array}{l}2 \times 45 \text {-min sessions per } \\
\text { week delivered over } 8-9 \\
\text { weeks. }\end{array}$ & $4 / 7$ \\
\hline
\end{tabular}




\begin{tabular}{|c|c|c|c|c|c|c|}
\hline Reference & Research design & $\begin{array}{l}\text { Intervention } \\
\text { approach }\end{array}$ & $\begin{array}{c}\text { Participant } \\
\text { number and age } \\
\text { (years;months) }\end{array}$ & $\begin{array}{c}\text { Summary of service } \\
\text { delivery }^{\mathrm{a}}\end{array}$ & $\begin{array}{l}\text { Summary of intervention } \\
\text { intensity }\end{array}$ & $\begin{array}{l}\text { Reporting of } \\
\text { intervention } \\
\text { intensity }^{b}\end{array}$ \\
\hline $\begin{array}{l}\text { Tyler, Gillon, Macrae } \\
\text { and Johnson (2011) }\end{array}$ & RCT & $\begin{array}{l}\text { Morphosyntax and } \\
\text { speech sound } \\
\text { intervention } \\
\text { compared with } \\
\text { phonological } \\
\text { awareness and } \\
\text { speech sound } \\
\text { intervention }\end{array}$ & $n=30(3 ; 10-5 ; 2)$ & $\begin{array}{l}\text { Group intervention } \\
\text { delivered by SLT } \\
\text { students }\end{array}$ & $\begin{array}{l}\text { Participants received } 2 \times \\
\text { blocks of intervention } \\
\text { separated by a 6-7 week } \\
\text { break. Each 6-week } \\
\text { block comprised } 2 \times 60 \text { - } \\
\text { min sessions per week, } \\
\text { for a total of } 24 \text { sessions } \\
\text { delivered over } 12 \text { weeks } \\
\text { of intervention. For the } \\
\text { morphosyntax } \\
\text { intervention, each } \\
\text { session comprised a } \\
\text { production dose of } 35- \\
45 \text { and an average } \\
\text { perception dose of } 75- \\
80 \text {. Dose was not } \\
\text { reported for the } \\
\text { phonological awareness } \\
\text { intervention. }\end{array}$ & $5 / 7$ \\
\hline Tyler and Lewis (2005) & $\begin{array}{l}\text { Retrospective } \\
\text { between-subjects } \\
\text { design }\end{array}$ & $\begin{array}{l}\text { Generic phonological } \\
\text { approach and } \\
\text { morphosyntax } \\
\text { intervention }\end{array}$ & $n=40(3 ; 0-5 ; 11)$ & $\begin{array}{l}\text { Individual and group } \\
\text { intervention delivered } \\
\text { by an SLT student in an } \\
\text { early childhood centre. }\end{array}$ & $\begin{array}{l}1 \times 30 \text {-min individual and } \\
1 \times 45 \text {-min group } \\
\text { session per week for } 24 \\
\text { weeks. }\end{array}$ & $4 / 7$ \\
\hline
\end{tabular}




\begin{tabular}{|c|c|c|c|c|c|c|}
\hline Reference & Research design & $\begin{array}{l}\text { Intervention } \\
\text { approach }\end{array}$ & $\begin{array}{c}\text { Participant } \\
\text { number and age } \\
\text { (years;months) }\end{array}$ & $\begin{array}{c}\text { Summary of service } \\
\text { delivery }^{\text {a }}\end{array}$ & $\begin{array}{l}\text { Summary of intervention } \\
\text { intensity }\end{array}$ & $\begin{array}{l}\text { Reporting of } \\
\text { intervention } \\
\text { intensity }^{b}\end{array}$ \\
\hline $\begin{array}{l}\text { Tyler, Lewis, Haskill and } \\
\text { Tolbert (2002) }\end{array}$ & Non-RCT & $\begin{array}{l}\text { Generic phonological } \\
\text { approach and } \\
\text { morphosyntax } \\
\text { intervention }\end{array}$ & $n=27(3 ; 0-5 ; 11)$ & $\begin{array}{l}\text { Individual and group } \\
\text { intervention delivered } \\
\text { by an SLT student in a } \\
\text { preschool. }\end{array}$ & $\begin{array}{l}1 \times 30 \text {-min individual and } \\
1 \times 45 \text {-min group } \\
\text { session per week for } 2 \times \\
12 \text {-week blocks } \\
\text { delivered over a school } \\
\text { year. For the } \\
\text { phonological } \\
\text { intervention, each } \\
\text { session comprised a } \\
\text { production dose of } 24- \\
32 \text { trials. For the } \\
\text { morphosyntax } \\
\text { intervention, each } \\
\text { session comprised a } \\
\text { production dose of } 20- \\
30 \text { trials. Both } \\
\text { interventions included } \\
\text { perceptual and/or } \\
\text { conceptual trials, but the } \\
\text { dose was not reported. }\end{array}$ & $4 / 7$ \\
\hline $\begin{array}{l}\text { Tyler, Lewis, Haskill and } \\
\text { Tolbert (2003a) }\end{array}$ & RCT & $\begin{array}{l}\text { Generic phonological } \\
\text { approach and } \\
\text { morphosyntax } \\
\text { intervention }\end{array}$ & $n=47(3 ; 0-5 ; 11)$ & $\begin{array}{l}\text { Individual and group } \\
\text { intervention delivered } \\
\text { by an SLT student in a } \\
\text { preschool. }\end{array}$ & $\begin{array}{l}1 \times 30 \text {-min individual and } \\
1 \times 45 \text {-min group } \\
\text { session per week for a } \\
\text { total of } 35-48 \text { sessions } \\
\text { delivered over } 24 \\
\text { weeks. For the } \\
\text { phonological } \\
\text { intervention, sessions } \\
\text { comprised between } 14 \\
\text { and } 60 \text { production trials. } \\
\text { For the morphosyntax } \\
\text { intervention, each } \\
\text { session comprised an } \\
\text { average } 23 \text { production } \\
\text { trials and an average } \\
75-80 \text { perception trials. }\end{array}$ & $6 / 7$ \\
\hline
\end{tabular}




\begin{tabular}{|c|c|c|c|c|c|c|}
\hline Reference & Research design & $\begin{array}{l}\text { Intervention } \\
\text { approach }\end{array}$ & $\begin{array}{c}\text { Participant } \\
\text { number and age } \\
\text { (years;months) }\end{array}$ & $\begin{array}{c}\text { Summary of service } \\
\text { delivery }^{\mathrm{a}}\end{array}$ & $\begin{array}{c}\text { Summary of intervention } \\
\text { intensity }\end{array}$ & $\begin{array}{c}\text { Reporting of } \\
\text { intervention } \\
\text { intensity }^{\mathbf{b}}\end{array}$ \\
\hline \multirow[t]{2}{*}{$\begin{array}{l}\text { Tyler, Lewis and Welch } \\
\quad(2003 b)\end{array}$} & $\begin{array}{l}\text { Study 1: RCT } \\
\text { (based on Tyler } \\
\quad \text { et al., 2003a) }\end{array}$ & $\begin{array}{l}\text { Study 1: Generic } \\
\text { phonological } \\
\text { approach and } \\
\text { morphosyntax } \\
\text { intervention }\end{array}$ & $\begin{array}{l}\text { Study } 1: n=20 \\
\quad(3 ; 0-5 ; 11)\end{array}$ & $\begin{array}{l}\text { Study 1: Individual and } \\
\text { group intervention } \\
\text { delivered by an SLT } \\
\text { student in a preschool. }\end{array}$ & $\begin{array}{l}\text { Study } 1 \text { : } 1 \times 30 \text {-min } \\
\text { individual and } 1 \times 45- \\
\text { min group session per } \\
\text { week for a total of } 24 \\
\text { weeks. For the } \\
\text { phonological } \\
\text { intervention, each } \\
\text { session comprised 24- } \\
32 \text { production trials. For } \\
\text { the morphosyntax } \\
\text { intervention, each } \\
\text { session comprised 20- } \\
30 \text { production trials. } \\
\text { Both interventions } \\
\text { included perceptual } \\
\text { and/or conceptual trials, } \\
\text { but the dose was not } \\
\text { reported. }\end{array}$ & $4 / 7$ \\
\hline & $\begin{array}{l}\text { Replication study: } \\
\text { RCT }\end{array}$ & $\begin{array}{l}\text { Replication study: As } \\
\text { in study } 1 \text {, above. }\end{array}$ & $\begin{array}{l}\text { Replication study: } n \\
\quad=20\left(\mathrm{M}_{\mathrm{age}}=4 ; 2\right)\end{array}$ & $\begin{array}{l}\text { Replication study: As in } \\
\text { study 1, above. }\end{array}$ & $\begin{array}{l}\text { Replication study: As in } \\
\text { study } 1 \text {, above. }\end{array}$ & $4 / 7$ \\
\hline $\begin{array}{l}\text { Tyler and Sandoval } \\
\text { (1994) }\end{array}$ & $\begin{array}{l}\text { MBD across } \\
\text { participants with } \\
\text { MPD }\end{array}$ & $\begin{array}{l}\text { Minimal pairs } \\
\text { compared with a } \\
\text { narrative } \\
\text { intervention } \\
\text { compared with a } \\
\text { combination of the } \\
\text { two approaches }\end{array}$ & $n=6(3 ; 6-4 ; 8)$ & $\begin{array}{l}\text { Individual intervention } \\
\text { delivered by an SLT in } \\
\text { a university clinic. }\end{array}$ & $\begin{array}{l}2-3 \times 45-\text { min sessions } \\
\text { per week for a total of } \\
24 \text { sessions delivered } \\
\text { over } 12 \text { weeks. One } \\
\text { participant terminated } \\
\text { intervention after } 6 \\
\text { weeks. }\end{array}$ & $5 / 7$ \\
\hline $\begin{array}{l}\text { Tyler and Watterson } \\
\text { (1991) }\end{array}$ & Non-RCT & $\begin{array}{l}\text { Modified cycles } \\
\text { compared with a } \\
\text { morphosyntax } \\
\text { intervention }\end{array}$ & $n=12(3 ; 7-5 ; 7)$ & $\begin{array}{l}\text { Group intervention } \\
\text { delivered by an SLT in } \\
\text { a university clinic. }\end{array}$ & $\begin{array}{l}2 \text { sessions per week for } 9 \\
\text { weeks. Each child } \\
\text { received a total of } \\
\text { approx. } 16 \text { sessions. }\end{array}$ & $4 / 7$ \\
\hline $\begin{array}{l}\text { Tyler, Williams and } \\
\text { Lewis (2006) }\end{array}$ & $\begin{array}{l}\text { Retrospective } \\
\text { between-subjects } \\
\text { design (based on } \\
\text { Tyler } \text { et al., } \\
\text { 2003a) }\end{array}$ & $\begin{array}{l}\text { Generic phonological } \\
\text { approach and } \\
\text { morphosyntax } \\
\text { intervention }\end{array}$ & $n=20(3 ; 0-5 ; 11)$ & $\begin{array}{l}\text { Individual and group } \\
\text { intervention delivered } \\
\text { by an SLT student at an } \\
\text { elementary school. }\end{array}$ & $\begin{array}{l}1 \times 30 \text {-min individual and } \\
1 \times 45 \text {-min group } \\
\text { session per week for a } \\
\text { total of } 24 \text { weeks. }\end{array}$ & $4 / 7$ \\
\hline
\end{tabular}




\begin{tabular}{|c|c|c|c|c|c|c|}
\hline Reference & Research design & $\begin{array}{l}\text { Intervention } \\
\text { approach }\end{array}$ & $\begin{array}{c}\text { Participant } \\
\text { number and age } \\
\text { (years;months) }\end{array}$ & $\begin{array}{c}\text { Summary of service } \\
\text { delivery }^{\mathrm{a}}\end{array}$ & $\begin{array}{c}\text { Summary of intervention } \\
\text { intensity }^{\mathrm{a}}\end{array}$ & $\begin{array}{l}\text { Reporting of } \\
\text { intervention } \\
\text { intensity }^{b}\end{array}$ \\
\hline $\begin{array}{l}\text { van Bysterveldt, Gillon } \\
\text { and Foster-Cohen } \\
\text { (2010) }\end{array}$ & $\begin{array}{l}\text { 'Multiple single- } \\
\text { subject repeated } \\
\text { measures } \\
\text { (duplicated AB) } \\
\text { design' (p. 323) }\end{array}$ & $\begin{array}{l}\text { Phonological } \\
\text { awareness } \\
\text { intervention } \\
\text { incorporating } \\
\text { speech errors }\end{array}$ & $n=10(4 ; 4-5 ; 5)$ & $\begin{array}{l}\text { Individual intervention } \\
\text { delivered by an SLT in } \\
\text { an early intervention } \\
\text { centre and by a parent at } \\
\text { home. }\end{array}$ & $\begin{array}{l}2 \times 6 \text {-week blocks } \\
\text { separated by a 6-week } \\
\text { break. Each block } \\
\text { comprised } 2 \times 20 \text {-min } \\
\text { sessions per week }(1 \times \\
\text { speech therapy session } \\
\text { and } 1 \times \text { 'learning } \\
\text { through computer' } \\
\text { session supporting } \\
\text { speech and language } \\
\text { therapy goals). } \\
\text { Production dose for the } \\
\text { speech therapy sessions } \\
\text { was described, but exact } \\
\text { dose unclear. In total, } \\
24 \text { intervention sessions } \\
\text { were delivered over } 18 \\
\text { weeks. }\end{array}$ & $5 / 7$ \\
\hline $\begin{array}{l}\text { van Bysterveldt, Gillon } \\
\text { and Foster-Cohen } \\
\text { (2014) }\end{array}$ & $\begin{array}{l}\text { Case study } \\
\text { (participant from } \\
\text { van Bysterveldt } \\
\text { et al., 2010) }\end{array}$ & $\begin{array}{l}\text { Phonological } \\
\text { awareness } \\
\text { intervention } \\
\text { incorporating } \\
\text { speech errors }\end{array}$ & $n=1$ 'Ben' $(5 ; 2)$ & $\begin{array}{l}\text { Individual intervention } \\
\text { delivered by an SLT in } \\
\text { an early intervention } \\
\text { centre and by a parent at } \\
\text { home. }\end{array}$ & $\begin{array}{l}2 \times 6 \text {-week blocks } \\
\text { separated by a } 6 \text {-week } \\
\text { break. Each block } \\
\text { comprised } 2 \times 20 \text {-min } \\
\text { sessions per week. }\end{array}$ & $4 / 7$ \\
\hline $\begin{array}{l}\text { Waters, Hawkes and } \\
\text { Burnett (1998) }\end{array}$ & Case study & $\begin{array}{l}\text { Psycholinguistic } \\
\text { approach }\end{array}$ & $n=1$ 'AG' $(5 ; 2)$ & $\begin{array}{l}\text { Individual intervention } \\
\text { delivered by an SLT. }\end{array}$ & $\begin{array}{l}2 \times \text { sessions per week } \\
\text { delivered over a 7- } \\
\text { month period. }\end{array}$ & $3 / 7$ \\
\hline
\end{tabular}




\begin{tabular}{|c|c|c|c|c|c|c|}
\hline Reference & Research design & $\begin{array}{l}\text { Intervention } \\
\text { approach }\end{array}$ & $\begin{array}{c}\text { Participant } \\
\text { number and age } \\
\text { (years;months) }\end{array}$ & $\begin{array}{c}\text { Summary of service } \\
\text { delivery }^{\mathrm{a}}\end{array}$ & $\begin{array}{c}\text { Summary of intervention } \\
\text { intensity }^{\mathrm{a}}\end{array}$ & $\begin{array}{c}\text { Reporting of } \\
\text { intervention } \\
\text { intensity }\end{array}$ \\
\hline Weiner (1981) & $\begin{array}{l}\text { MBD across } \\
\text { behaviours }\end{array}$ & Minimal pairs & $n=2(4 ; 4-4 ; 10)$ & $\begin{array}{l}\text { Individual intervention } \\
\text { delivered by an SLT in } \\
\text { a university clinic. }\end{array}$ & $\begin{array}{l}3 \times 60 \text {-min sessions per } \\
\text { week, for a total of } \\
\text { either } 5 \text { or } 13 \text { treatment } \\
\text { sessions. Each session } \\
\text { comprised between } 40 \\
\text { and } 80 \text { production trials, } \\
\text { for a cumulative } \\
\text { intervention intensity of } \\
400 \text { or } 540 \text { production } \\
\text { trials for each } \\
\text { participant. }\end{array}$ & $6 / 7$ \\
\hline Williams (1991) & $\begin{array}{l}\text { MBD across } \\
\text { behaviours }\end{array}$ & $\begin{array}{l}\text { Complexity } \\
\text { approach: Clusters } \\
\text { associated with } \\
\text { least knowledge }\end{array}$ & $n=9(3 ; 8-5 ; 9)$ & $\begin{array}{l}\text { Individual intervention } \\
\text { delivered by an SLT in } \\
\text { a university clinic. }\end{array}$ & $\begin{array}{l}\text { Up to } 21 \text { sessions, each } \\
\text { comprising } 100 \\
\text { production trials. }\end{array}$ & $3 / 7$ \\
\hline Williams (1993) & Case study & $\begin{array}{l}\text { Complexity } \\
\text { approach: } \\
\text { Modified maximal } \\
\text { oppositions }\end{array}$ & $\begin{array}{l}n=1 \text { 'Michael' } \\
\quad(6 ; 11)\end{array}$ & $\begin{array}{l}\text { Individual intervention } \\
\text { delivered by an SLT at } \\
\text { school. }\end{array}$ & $\begin{array}{l}13 \times 45 \text {-min sessions } \\
\text { completed, each } \\
\text { comprising } 100 \\
\text { production trials. }\end{array}$ & $4 / 7$ \\
\hline Williams (2000) & Case study & $\begin{array}{l}\text { Multiple oppositions } \\
\text { followed by } \\
\text { minimal pairs } \\
\text { and/or naturalistic } \\
\text { speech } \\
\text { intelligibility } \\
\text { training }\end{array}$ & $n=10(4 ; 0-6 ; 5)$ & $\begin{array}{l}\text { Individual intervention } \\
\text { delivered by an SLT in } \\
\text { a university clinic. }\end{array}$ & $\begin{array}{l}2 \times 30 \text {-min sessions per } \\
\text { week, for a total of } \\
\text { between } 26 \text { and } 105 \\
\text { sessions (average }=60 \text { ) } \\
\text { delivered over } 2-5 \\
\text { university semesters } \\
\text { (average }=3.4 \text { ). } \\
\text { Intervention was } \\
\text { provided from referral } \\
\text { to discharge. }\end{array}$ & $5 / 7$ \\
\hline Williams (2005) & $\begin{array}{l}\text { MBD across } \\
\text { behaviours }\end{array}$ & $\begin{array}{l}\text { Multiple oppositions } \\
\text { compared with } \\
\text { minimal pairs }\end{array}$ & $n=1$ 'Jane' $(6 ; 5)$ & $\begin{array}{l}\text { Individual intervention } \\
\text { delivered by an SLT in } \\
\text { a university clinic. }\end{array}$ & $\begin{array}{l}42 \times 30 \text {-min sessions }(21 \\
\text { sessions per } \\
\text { intervention approach) } \\
\text { each comprising } 80-100 \\
\text { production trials. }\end{array}$ & $4 / 7$ \\
\hline
\end{tabular}




\begin{tabular}{|c|c|c|c|c|c|c|}
\hline Reference & Research design & $\begin{array}{l}\text { Intervention } \\
\text { approach }\end{array}$ & $\begin{array}{c}\text { Participant } \\
\text { number and age } \\
\text { (years;months) }\end{array}$ & $\begin{array}{c}\text { Summary of service } \\
\text { delivery }^{\mathrm{a}}\end{array}$ & $\begin{array}{c}\text { Summary of intervention } \\
\text { intensity }^{\mathrm{a}}\end{array}$ & $\begin{array}{l}\text { Reporting of } \\
\text { intervention } \\
\text { intensity }^{b}\end{array}$ \\
\hline \multirow[t]{3}{*}{ Williams (2012) } & $\begin{array}{l}\text { Study 1: Combined } \\
\text { MBD across } \\
\text { behaviours and } \\
\text { participants }\end{array}$ & $\begin{array}{l}\text { Study 1: Multiple } \\
\text { oppositions }\end{array}$ & $\begin{array}{l}\text { Study } 1: n=14 \\
\quad(4 ; 0-6 ; 0)\end{array}$ & $\begin{array}{l}\text { Study 1: Individual } \\
\text { intervention delivered } \\
\text { by an SLT in a } \\
\text { university clinic. }\end{array}$ & $\begin{array}{l}\text { Study } 1: 2 \times 30-\text { min } \\
\text { sessions per week for a } \\
\text { total of } 20-42 \text { sessions, } \\
\text { each comprising an } \\
\text { average of } 65.39 \\
\text { production trials. } \\
\text { Cumulative intervention } \\
\text { intensity ranged from } \\
1404 \text { to } 3708 \text { per } \\
\text { participant (average }= \\
2455.59) .\end{array}$ & $6 / 7$ \\
\hline & $\begin{array}{l}\text { Study 2: MBD } \\
\text { across behaviours }\end{array}$ & $\begin{array}{l}\text { Study 2: Multiple } \\
\text { oppositions } \\
\text { compared with } \\
\text { minimal pairs }\end{array}$ & $\begin{array}{l}\text { Study } 2: n=4 \\
(4 ; 6-6 ; 5)\end{array}$ & $\begin{array}{l}\text { Study 2: Individual } \\
\text { intervention delivered } \\
\text { by an SLT in a } \\
\text { university clinic. }\end{array}$ & $\begin{array}{l}\text { Study } 2: 2 \times 30-\text { min } \\
\text { sessions per week for a } \\
\text { total of } 12-44 \\
\text { sessions, each } \\
\text { comprising an average } \\
\text { of } 78.99 \text { production } \\
\text { trials. Cumulative } \\
\text { intervention intensity } \\
\text { ranged from } 1364 \text { to } \\
\text { 3008 per participant } \\
\text { (average }=2499.25 \text { ). }\end{array}$ & $6 / 7$ \\
\hline & $\begin{array}{l}\text { Study 3: MBD } \\
\quad \text { across behaviours }\end{array}$ & $\begin{array}{l}\text { Study 3: Computer- } \\
\text { based generic } \\
\text { phonological } \\
\text { approach } \\
\text { compared with } \\
\text { minimal pairs }\end{array}$ & $\begin{array}{c}\text { Study } 3: n=4 \\
(3 ; 7-4 ; 9)\end{array}$ & $\begin{array}{l}\text { Study 3: Individual } \\
\text { intervention delivered } \\
\text { by an SLT in a } \\
\text { university clinic. }\end{array}$ & $\begin{array}{l}\text { Study } 3: 2 \times 30 \text {-min } \\
\text { sessions per week for a } \\
\text { total of 5-17 sessions, } \\
\text { each comprising an } \\
\text { average of } 51.56 \\
\text { production trials. } \\
\text { Cumulative intervention } \\
\text { intensity ranged from } \\
186 \text { to } 1015 \text { per } \\
\text { participant (average }= \\
\text { 529.5). }\end{array}$ & $6 / 7$ \\
\hline
\end{tabular}




\begin{tabular}{|c|c|c|c|c|c|c|}
\hline Reference & Research design & $\begin{array}{l}\text { Intervention } \\
\text { approach }\end{array}$ & $\begin{array}{c}\text { Participant } \\
\text { number and age } \\
\text { (years;months) }\end{array}$ & $\begin{array}{c}\text { Summary of service } \\
\text { delivery }^{\mathrm{a}}\end{array}$ & $\begin{array}{l}\text { Summary of intervention } \\
\text { intensity }\end{array}$ & $\begin{array}{c}\text { Reporting of } \\
\text { intervention } \\
\text { intensity }^{b}\end{array}$ \\
\hline $\begin{array}{l}\text { Wolfe, Blocker and } \\
\text { Prater (1988) }\end{array}$ & Case study & $\begin{array}{l}\text { Generic phonological } \\
\text { approach }\end{array}$ & $n=2(3 ; 5-4 ; 7)$ & $\begin{array}{l}\text { Individual intervention } \\
\text { delivered by an SLT. }\end{array}$ & $\begin{array}{l}\text { Intervention delivered over } \\
5 \text { or } 15 \text { months for each } \\
\text { participant, with each } \\
\text { session comprising } 100 \\
\text { production trials. }\end{array}$ & $3 / 7$ \\
\hline $\begin{array}{l}\text { Wolfe, Presley and } \\
\text { Mesaris (2003) }\end{array}$ & RCT & $\begin{array}{l}\text { Traditional multiple } \\
\text { phonemic } \\
\text { approach, with or } \\
\text { without speech } \\
\text { perception } \\
\text { training (SAILS) }\end{array}$ & $n=9(3 ; 5-4 ; 2)$ & $\begin{array}{l}\text { Individual intervention } \\
\text { delivered by an SLT } \\
\text { assistant or SLT } \\
\text { student. }\end{array}$ & $\begin{array}{l}\text { 30-min sessions delivered } \\
\text { 'biweekly' (p. } 284, \\
\text { coded as unclear), for } \\
\text { an average of } 11 \\
\text { sessions (range }=8-17 \text { ) } \\
\text { delivered over an } \\
\text { academic quarter. }\end{array}$ & $4 / 7$ \\
\hline $\begin{array}{l}\text { Wren and Roulstone } \\
\text { (2008) }\end{array}$ & RCT & $\begin{array}{l}\text { Table-top and } \\
\text { computer-based } \\
\text { intervention using } \\
\text { a psycholinguistic } \\
\text { framework }\end{array}$ & $n=33(4 ; 2-7 ; 10)$ & $\begin{array}{l}\text { Individual intervention } \\
\text { delivered by an SLT } \\
\text { and an assistant at } \\
\text { school. }\end{array}$ & $\begin{array}{l}1 \times 30 \text {-min session per } \\
\text { week with the SLT and } \\
2 \times 30 \text {-min sessions per } \\
\text { week with the assistant } \\
\text { for } 8 \text { weeks, for a total } \\
\text { of between } 18 \text { and } 24 \\
\text { sessions. }\end{array}$ & $5 / 7$ \\
\hline $\begin{array}{l}\text { Yoder, Camarata and } \\
\quad \text { Gardner (2005) }\end{array}$ & RCT & $\begin{array}{l}\text { Broad target speech } \\
\quad \text { recasts }\end{array}$ & $\begin{array}{l}n=52\left(\mathrm{M}_{\text {age }}=3.65\right. \\
\text { years })\end{array}$ & $\begin{array}{l}\text { Individual intervention } \\
\text { delivered by a } \\
\text { researcher. }\end{array}$ & $\begin{array}{c}3 \times 30 \text {-min sessions per } \\
\text { week delivered over a } \\
6 \text {-month period. Each } \\
\text { session comprised a } \\
\text { perceptual dose of } 4 \\
\text { recasts per minute } \\
\text { (coded as } \text { unclear) }\end{array}$ & $5 / 7$ \\
\hline
\end{tabular}




\begin{tabular}{|c|c|c|c|c|c|c|}
\hline Reference & Research design & $\begin{array}{c}\text { Intervention } \\
\text { approach }\end{array}$ & $\begin{array}{c}\text { Participant } \\
\text { number and age } \\
\text { (years;months) }\end{array}$ & $\begin{array}{c}\text { Summary of service } \\
\text { delivery }^{\mathrm{a}}\end{array}$ & $\begin{array}{c}\text { Summary of intervention } \\
\text { intensity }\end{array}$ & $\begin{array}{c}\text { Reporting of } \\
\text { intervention } \\
\text { intensity }\end{array}$ \\
\hline $\begin{array}{l}\text { Yoder, Camarata and } \\
\text { Woynaroski (2016) }\end{array}$ & RCT & $\begin{array}{l}\text { Broad target speech } \\
\text { recasts compared } \\
\text { with modified } \\
\text { cycles }\end{array}$ & $\begin{array}{l}n=51\left(\mathrm{M}_{\text {age }}=6.5\right. \\
\text { and } 7.8 \text { years for } \\
\text { each group })\end{array}$ & $\begin{array}{l}\text { Individual intervention } \\
\text { delivered by an SLT at } \\
\text { school. }\end{array}$ & $\begin{array}{l}2 \times 60 \text {-min sessions per } \\
\text { week for } 6 \text { months. For } \\
\text { the broad target speech } \\
\text { recasts intervention, } \\
\text { each session comprised } \\
\text { an average perceptual } \\
\text { dose of } 4.07 \text { recasts per } \\
\text { minute (p. } 452 \text {, coded as } \\
\text { unclear). For the } \\
\text { modified cycles } \\
\text { approach, SLTs } \\
\text { requested the participant } \\
\text { imitate productions } 2.25 \\
\text { times per minute }(\mathrm{p} . \\
\text { 452, coded as unclear). }\end{array}$ & $5 / 7$ \\
\hline Young (1987) & $\begin{array}{l}\text { MBD across } \\
\text { behaviours }\end{array}$ & $\begin{array}{l}\text { Backward chaining } \\
\text { with rebuses }\end{array}$ & $n=2(4 ; 4-4 ; 5)$ & $\begin{array}{l}\text { Individual intervention } \\
\text { delivered by an SLT in } \\
\text { a university clinic. }\end{array}$ & $\begin{array}{l}2 \times \text { sessions per week, } \\
\text { each comprising } 50 \\
\text { production trials. }\end{array}$ & $3 / 7$ \\
\hline
\end{tabular}

${ }^{a}$ Not all studies provided details about all elements of service delivery or intervention intensity. Absence of this information in the table reflects an absences of this information in the published paper. Note that home practice is not included in descriptions of intervention intensity (for more detail on the intervention intensity delivered by home practice, see Sugden, Baker, Munro, \& Williams, 2016).

${ }^{b}$ Studies received a score out of 7, with one point allocated for reporting each of the following components of intervention intensity: dose, dose form, dose frequency, session duration, total intervention duration (in weeks or months), total intervention duration (in sessions), and cumulative intervention intensity.

${ }^{c}$ Reviews were not rated for reporting of intervention intensity, as they aim to collate previously-published studies rather than report on a specific investigation of a phonological intervention.

${ }^{\mathrm{d}}$ The term generic phonological approach was used when an explicit name was not provided for an intervention delivered to children with a phonological delay/disorder/impairment. 


\section{References}

ABRAHAM, S. 1993. Differential treatment of phonological disability in children with impaired hearing who were trained orally. American Journal of Speech-Language

Pathology, 20, 23-30.

ADAMS, C., NIGHTINGALE, C., HESKETH, A. and HALL, R. 2000. Targeting metaphonological ability in intervention for children with developmental phonological disorders. Child Language Teaching and Therapy, 16, 285-299.

ALLEN, M. M. 2013. Intervention efficacy and intensity for children with speech sound disorder. Journal of Speech, Language, and Hearing Research, 56, 865-877.

ALMOST, D. and ROSENBAUM, P. 1998. Effectiveness of speech intervention for phonological disorders: A randomized controlled trial. Developmental Medicine \& Child Neurology, 40, 319-325.

BAGETTI, T., CERON, M. I., MOTA, H. B. and KESKE-SOARES, M. 2012. Mudanças fonológicas após aplicação de abordagem terapêutica baseada em traços distintivos no tratamento do desvio fonológico [Phonological changes after the application of therapy approach based on distinctive features in the treatment of phonological disorder]. Jornal da Sociedade Brasileira de Fonoaudiologia, 24, 282-287.

BAKER, E. and MCLEOD, S. 2004. Evidence-based management of phonological impairment in children. Child Language Teaching and Therapy, $20,261-285$.

BAKER, E. and MCLEOD, S. 2011. Evidence-based practice for children with speech sound disorders: Part 1 narrative review. Language, Speech, and Hearing Services in Schools, 42, 102-139.

BARBERENA, L., KESKE-SOARES, M., CERVI, T. and BRANDÃO, M. 2014. Treatment model in children with speech disorders and its therapeutic efficiency. International Archives of Otorhinolaryngology, 18, 283-288.

BARLOW, J. A. 2005. Phonological change and the representation of consonant clusters in Spanish: A case study. Clinical Linguistics \& Phonetics, $19,659-679$.

BEDORE, L. M., LEONARD, L. B. and GANDOUR, J. 1994. The substitution of a click for sibilants: A case study. Clinical Linguistics \& Phonetics, 8, $283-293$.

BELLON-HARN, M. L., CREDEUR-PAMPOLINA, M. E. and LEBOEUF, L. 2013. Scaffolded-language intervention: Speech production outcomes. Communication Disorders Quarterly, 34, 120-132. 
BERNHARDT, B. 1992. The application of nonlinear phonological theory to intervention with one phonologically disordered child. Clinical Linguistics \& Phonetics, 6, 283-

316.

BERNHARDT, B. and MAJOR, E. M. 2005. Speech, language and literacy skills 3 years late: A follow-up study of early phonological and metaphonological intervention. International Journal of Language \& Communication Disorders, 40, 1-27.

BLACHE, S. E., PARSONS, C. L. and HUMPHREYS, J. M. 1981. A minimal-word-pair model for teaching the linguistic significance of distinctive feature properties. Journal of Speech and Hearing Disorders, 46, 291-296.

BOWEN, C. and CUPPLES, L. 1998. A tested phonological therapy in practice. Child Language Teaching and Therapy, 14, 29-50.

BOWEN, C. and CUPPLES, L. 1999a. Parents and children together (PACT): A collaborative approach to phonological therapy. International Journal of Language \& Communication Disorders, 34, 35-55.

BOWEN, C. and CUPPLES, L. 1999b. A phonological therapy in depth: A reply to commentaries. International Journal of Language \& Communication Disorders, 34, 6583.

BROEN, P. A. and WESTMAN, M. J. 1990. Project parent: A preschool speech program implemented through parents. Journal of Speech and Hearing Disorders, 55, 495502.

BROOMFIELD, J. and DODD, B. 2011. Is speech and language therapy effective for children with primary speech and language impairment? Report of a randomized control trial. International Journal of Language \& Communication Disorders, 46, 628-640.

BRYAN, A. and HOWARD, D. 1992. Frozen phonology thawed: The analysis and remediation of a developmental disorder of real word phonology. International Journal of Language \& Communication Disorders, 27, 343-365.

CAMARATA, S. M. 1993. The application of naturalistic conversation training to speech production in children with speech disabilities. Journal of Applied Behavior Analysis, 26, 173-182. 
CERON, M. I. and KESKE-SOARES, M. 2013. Mudanças fonológicas obtidas no tratamento pelo modelo de oposições múltiplas [Phonological changes obtained in the treatment based on the multiple oppositions approach]. Revista CEFAC, 15, 314-323.

CERON, M. I., KESKE-SOARES, M., DE FREITAS, G. P. and GUBIANI, M. B. 2010. Mudanças fonológicas obtidas no tratamento de sujeitos comparando diferentes modelos de terapia [Phonological changes obtained in the treatment of subjects comparing different therapy models]. Pró-Fono Revista de Atualização Científica, $22,549-554$.

CHECALIN, M. A., GHISLENI, M. R. L., FERREIRA-GONÇALVES, G., KESKE-SOARES, M. and MOTA, H. B. 2010. A regressão observada no tratamento do desvio fonológico [Relapse observed in the treatment of phonological disorder]. Pró-Fono Revista de Atualização Científica, 22, 363-6.

CONTURE, E. G., LOUKO, L. J. and EDWARDS, M. L. 1993. Simultaneously treating stuttering and disordered phonology in children: Experimental treatment, preliminary findings. American Journal of Speech-Language Pathology, 2, 72-81.

CROSBIE, S., HOLM, A. and DODD, B. 2005. Intervention for children with severe speech disorder: A comparison of two approaches. International Journal of Language \& Communication Disorders, 40, 467-491.

CROSBIE, S., PINE, C., HOLM, A. and DODD, B. 2006. Treating Jarrod: A core vocabulary approach. International Journal of Speech-Language Pathology, 8, 316-321.

CULATTA, B., SETZER, L. A. and HORN, D. 2005. Meaning-based intervention for a child with speech and language disorders. Topics in Language Disorders, 25, 388401.

CUMMINGS, A. E. and BARLOW, J. A. 2011. A comparison of word lexicality in the treatment of speech sound disorders. Clinical Linguistics \& Phonetics, 25, 265-286.

DEAN, E. C., HOWELL, J., WATERS, D. and REID, J. 1995. Metaphon: A metalinguistic approach to the treatment of phonological disorder in children. Clinical Linguistics \& Phonetics, 9, 1-19.

DENNE, M., LANGDOWN, N., PRING, T. and ROY, P. 2005. Treating children with expressive phonological disorders: Does phonological awareness therapy work in the clinic? International Journal of Language \& Communication Disorders, 40, 493-504. 
DERAKHSHANDEH, F., NIKMARAM, M., HOSSEINABAD, H. H., MEMARZADEH, M., TAHERI, M., OMRANI, M., JALAIE, S., BIJANKHAN, M. and SELL, D.

2016. Speech characteristics after articulation therapy in children with cleft palate and velopharyngeal dysfunction - A single case experimental design. International Journal of Pediatric Otorhinolaryngology, 86, 104-13.

DINNSEN, D. A., CHIN, S. B. and ELBERT, M. 1992. On the lawfulness of change in phonetic inventories. Lingua, 86, 207-222.

DODD, B. and BARKER, R. 1990. The efficacy of utilizing parents and teachers as agents of therapy for children with phonological disorders. Australian Journal of Human Communication Disorders, 18, 29-44.

DODD, B. and BRADFORD, A. 2000. A comparison of three therapy models for children with different types of developmental phonological disorder. International Journal of Language \& Communication Disorders, 35, 189-209.

DODD, B., CROSBIE, S., MCINTOSH, B., HOLM, A., HARVEY, C., LIDDY, M., FONTYNE, K., PINCHIN, B. and RIGBY, H. 2008. The impact of selecting different contrasts in phonological therapy. International Journal of Speech-Language Pathology, 10, 334-345.

DODD, B. and IACANO, T. 1989. Phonological disorders in children: Changes in phonological process use during treatment. International Journal of Language \& Communication Disorders, 24, 333-351.

DONICHT, G., PAGLIARIN, K. C., MOTA, H. B. and KESKE-SOARES, M. 2011. O tratamento com os róticos e a generalização obtida em dois modelos de terapia fonológica [The treatment with rothics and generalization obtained in two models of phonological therapy]. Jornal da Sociedade Brasileira de Fonoaudiologia, 23, $71-76$.

DUNN, C. and BARRON, C. 1982. A treatment program for disordered phonology: Phonetic and linguistic considerations. Language, Speech, and Hearing Services in Schools, 13, 100-109.

EIKESETH, S. and NESSET, R. 2003. Behavioral treatment of children with phonological disorder: The efficacy of vocal imitation and sufficient-response-exemplar training. Journal of Applied Behavior Analysis, 36, 325-337. 
EISERMAN, W. D., MCCOUN, M. and ESCOBAR, C. M. 1990. A cost-effectiveness analysis of two alternative program models for serving speech-disordered preschoolers. Journal of Early Intervention, 14, 297-317.

EISERMAN, W. D., WEBER, C. and MCCOUN, M. 1992. Two alternative program models for serving speech-disordered preschoolers: A second year follow-up. Journal of Communication Disorders, 25, 77-106.

ELBERT, M. 1983. Case study of phonological acquisition. Topics in Language Disorders, 3, 1-9.

ELBERT, M., DINNSEN, D. A., SWARTZLANDER, P. and CHIN, S. B. 1990. Generalization to conversational speech. Journal of Speech and Hearing Disorders, 55, 694699.

ELBERT, M., POWELL, T. W. and SWARTZLANDER, P. 1991. Toward a technology of generalization: How many exemplars are sufficient? Journal of Speech and Hearing Research, 34, 81-87.

FEEHAN, A., FRANCIS, C., BERNHARDT, B. M. and COLOZZO, P. 2015. Phonological and morphosyntactic intervention for a twin pair. Child Language Teaching and Therapy, 31, 53-69.

FEY, M., CLEAVE, P. L., RAVIDA, A. I., LONG, S. H., DEJMAL, A. E. and EASTON, D. L. 1994. Effects of grammar facilitation on the phonological performance of children with speech and language impairments. Journal of Speech and Hearing Research, 37, 594-607.

FEY, M. and STALKER, C. H. 1986. A hypothesis-testing approach to treatment of a child with an idiosyncratic (morpho)phonological system. Journal of Speech and Hearing Disorders, 51, 309-324.

FLINT, C. B. and COSTELLO INGHAM, J. 2005. Pretreatment stimulability and percentage of consonants correct as predictors of across-phoneme generalization. Contemporary Issues in Communication Sciences and Disorders, 32, 53-63.

FORREST, K., DINNSEN, D. A. and ELBERT, M. 1997. Impact of substitution patterns on phonological learning by misarticulating children. Clinical Linguistics \& Phonetics, 11, 63-76.

FORREST, K. and ELBERT, M. 2001. Treatment for phonologically disordered children with variable substitution patterns. Clinical Linguistics \& Phonetics, 15, 41-45. 
FORREST, K., ELBERT, M. and DINNSEN, D. A. 2000. The effect of substitution patterns on phonological treatment outcomes. Clinical Linguistics \& Phonetics, 14, 519531.

GIBBON, F., SHOCKEY, L. and REID, J. 1992. Description and treatment of abnormal vowels in a phonologically disordered child. Child Language Teaching and Therapy, $8,30-59$.

GIERUT, J. A. 1989. Maximal opposition approach to phonological treatment. Journal of Speech and Hearing Disorders, 54, 9-19.

GIERUT, J. A. 1990. Differential learning of phonological oppositions. Journal of Speech and Hearing Research, 33, 540-549.

GIERUT, J. A. 1991. Homonymy in phonological change. Clinical Linguistics \& Phonetics, 5, 119-137.

GIERUT, J. A. 1992. The conditions and course of clinically induced phonological change. Journal of Speech and Hearing Research, 35, $1049-1063$.

GIERUT, J. A. 1996. An experimental test of phonemic cyclicity. Journal of Child Language, 23, 81-102.

GIERUT, J. A. 1998. Natural domains of cyclicity in phonological acquisition. Clinical Linguistics \& Phonetics, 12, 481-499.

GIERUT, J. A. 1999. Syllable onsets: Clusters and adjuncts in acquisition. Journal of Speech, Language, and Hearing Research, 42, 708-726.

GIERUT, J. A. and CHAMPION, A. H. 1999. Interacting error patterns and their resistance to treatment. Clinical Linguistics \& Phonetics, 13, 421-431.

GIERUT, J. A. and CHAMPION, A. H. 2000. Ingressive substitutions: Typical or atypical phonological pattern? Clinical Linguistics \& Phonetics, $14,603-617$.

GIERUT, J. A. and CHAMPION, A. H. 2001. Syllable onsets II: Three-element clusters in phonological treatment. Journal of Speech, Language, and Hearing Research, 44, 886-904.

GIERUT, J. A., ELBERT, M. and DINNSEN, D. A. 1987. A functional analysis of phonological knowledge and generalisation learning in misarticulating children. Journal of Speech and Hearing Research, 30, 462-479.

GIERUT, J. A. and MORRISETTE, M. L. 1996. Triggering a principle of phonemic acquisition. Clinical Linguistics \& Phonetics, 10, 15-30.

GIERUT, J. A. and MORRISETTE, M. L. 2010. Phonological learning and lexicality of treated stimuli. Clinical Linguistics \& Phonetics, 24, 122-140.

GIERUT, J. A. and MORRISETTE, M. L. 2012a. Age of word acquisition effects in treatment of children with phonological delays. Applied Psycholinguistics, 33, 121-144. 
GIERUT, J. A. and MORRISETTE, M. L. 2012b. Density, frequency and the expressive phonology of children with phonological delay. Journal of Child Language, 39, 804834.

GIERUT, J. A. and MORRISETTE, M. L. 2014. How to meet the neighbors: Modality effects on phonological generalization. Clinical Linguistics \& Phonetics, $28,477-492$.

GIERUT, J. A. and MORRISETTE, M. L. 2015. Dense neighborhoods and mechanisms of learning: evidence from children with phonological delay. Journal of Child Language, 42, 1036-72.

GIERUT, J. A., MORRISETTE, M. L. and CHAMPION, A. H. 1999. Lexical constraints in phonological acquisition. Journal of Child Language, $26,261-294$.

GIERUT, J. A., MORRISETTE, M. L., HUGHES, M. T. and ROWLAND, S. 1996. Phonological treatment efficacy and developmental norms. Language, Speech and Hearing Services in Schools, 27, 215-230.

GIERUT, J. A., MORRISETTE, M. L. and ZIEMER, S. M. 2010. Nonwords and generalization in children with phonological disorders. American Journal of SpeechLanguage Pathology, 19, 167-177.

GIERUT, J. A. and NEUMANN, H. J. 1992. Teaching and learning /8/: A non-confound. Clinical Linguistics \& Phonetics, 6, 191-200.

GILDERSLEEVE-NEUMANN, C. and GOLDSTEIN, B. A. 2015. Cross-linguistic generalization in the treatment of two sequential Spanish-English bilingual children with speech sound disorders. International Journal of Speech-Language Pathology, 17, 26-40.

GILLON, G. T. 2000. The efficacy of phonological awareness intervention for children with spoken language impairment. Language, Speech, and Hearing Services in Schools, 31, 126-141.

GILLON, G. T. 2005. Facilitating phoneme awareness development in 3- and 4-year-old children with speech impairment. Language, Speech, and Hearing Services in Schools, 36, 308-324.

GIROLAMETTO, L., PEARCE, P. S. and WEITZMAN, E. 1997. Effects of lexical intervention on the phonology of late talkers. Journal of Speech, Language, and Hearing Research, 40, 338-348 
GLASPEY, A. and MACLEOD, A. A. 2010. A multi-dimensional approach to gradient change in phonological acquisition: a case study of disordered speech development.

Clinical Linguistics \& Phonetics, 24, 283-99.

GLASPEY, A. and STOEL-GAMMON, C. 2005. Dynamic assessment in phonological disorders: The Scaffolding Scale of Stimulability. Topics in Language Disorders, 25, 220-230.

GLASPEY, A. and STOEL-GAMMON, C. 2007. A dynamic approach to phonological assessment. International Journal of Speech-Language Pathology, 9, $286-296$.

GLOGOWSKA, M., ROULSTONE, S., ENDERBY, P. and PETERS, T. J. 2000. Randomised controlled trial of community based speech and language therapy in preschool children. British Medical Journal, 321, 1-5.

GOLDSTEIN, B. A. 1996. The role of stimulability in the assessment and treatment of spanish-speaking children. Journal of Communication Disorders, $29,299-314$.

GORDON-BRANNAN, M., HODSON, B. W. and WYNNE, M. K. 1992. Remediating unintelligible utterances of a child with a mild hearing loss. American Journal of Speech-Language Pathology, 1, 28-38.

GRAWBURG, M. and RVACHEW, S. 2007. Phonological awareness intervention for children with speech sound disorders. Canadian Journal of Speech-Language Pathology and Audiology, 31, 19-26.

GRUNWELL, P. and DIVE, D. 1988. Treating 'cleft palate speech': Combining phonological techniques with traditional articulation therapy. Child Language Teaching and Therapy, 4, 193-210.

GRUNWELL, P. and RUSSELL, J. 1990. A phonological disorder in an English-speaking child: A case study. Clinical Linguistics \& Phonetics, 4, $29-38$.

GRUNWELL, P., YAVAS, M., RUSSELL, J. and LE MAISTRE, H. 1988. Developing a phonological system: A case study. Child Language Teaching and Therapy, 4, $142-$ 153.

HARBERS, H. M., PADEN, E. P. and HALLE, J. W. 1999. Phonological awareness and production: Changes during intervention. Language, Speech and Hearing Services in Schools, 30, 50-60. 
HART, S. and GONZALEZ, L. 2010. The effectiveness of using communication-centered intervention to facilitate phonological learning in young children. Communication Disorders Quarterly, 32, 13-25.

HERMAN, R., FORD, K., THOMAS, J., OYEBADE, N., BENNETT, D. and DODD, B. 2015. Evaluation of core vocabulary therapy for deaf children: Four treatment case studies. Child Language Teaching and Therapy, 31, 221-235.

HESKETH, A., ADAMS, C., NIGHTINGALE, C. and HALL, R. 2000. Phonological awareness therapy and articulatory training approaches for children with phonological disorders: A comparative outcome study. International Journal of Language \& Communication Disorders, 35, $337-354$.

HESKETH, A., DIMA, E. and NELSON, V. 2007. Teaching phoneme awareness to pre-literate children with speech disorder: A randomized controlled trial. International Journal of Language \& Communication Disorders, 42, $251-271$.

HODSON, B. W. 1983. A facilitative approach for remediation of a child's profoundly unintelligible phonological system. Topics in Language Disorders, 3, 24-34.

HODSON, B. W., CHIN, L., REDMOND, B. and SIMPSON, R. 1983. Phonological evaluation and remediation of speech deviations of a child with a repaired cleft palate: a case study. Journal of Speech and Hearing Disorders, 48, 93-98.

HODSON, B. W., NONOMURA, C. W. and ZAPPIA, M. J. 1989. Phonological disorders: Impact on academic performance? Seminars in Speech and Language, 10, 252259.

HOFFMAN, P. R., NORRIS, J. A. and MONJURE, J. 1990. Comparison of process targeting and whole language treatments for phonologically delayed preschool children. Language, Speech, and Hearing Services in Schools, 21, 102-109.

HOLM, A. and DODD, B. 1999. An intervention case study of a bilingual child with phonological disorder. Child Language Teaching and Therapy, 15, 139-158.

HOLM, A. and DODD, B. 2001. Comparison of cross-language generalisation following speech therapy. Folia Phoniatrica et Logopaedica, 53, 166-172.

HOLM, A., DODD, B. and OZANNE, A. 1997. Efficacy of intervention for a bilingual child making articulation and phonological errors. The International Journal of Bilingualism, 1, 55-69.

JARVIS, J. 1989. Taking a Metaphon approach to phonological development: A case study. Child Language Teaching and Therapy, 5, 16-32. 
KESKE-SOARES, M., BRANCALIONI, A. R., MARINI, C., PAGLIARIN, K. C. and CERON, M. I. 2008. Eficácia da terapia para desvios fonológicos com diferentes modelos terapêuticos \{Therapy effectiveness for phonological disorders with different therapeutic approaches\}. Pró-Fono Revista de Atualização Científica, $20,153-9$.

KLEIN, E. S. 1996. Phonological/traditional approaches to articulation therapy: A retrospective group comparison. Language, Speech, and Hearing Services in Schools, 27, 314-323.

LANCASTER, G., KEUSCH, S., LEVIN, A., PRING, T. and MARTIN, S. 2010. Treating children with phonological problems: Does an eclectic approach to therapy work? International Journal of Language \& Communication Disorders, 45, 174-181.

LAW, J., GARRETT, Z. and NYE, C. 2004. The efficacy of treatment for children with developmental speech and language delay/disorder: A meta-analysis. Journal of Speech, Language, and Hearing Research, 47, 924-943.

LEAHY, J. and DODD, B. 1987. The development of disordered phonology: A case study. Language and Cognitive Processes, 2, 115-132.

LEITE, R. A., WERTZNER, H. F., GONCALVES, I. C., MAGLIARO, F. C. and MATAS, C. G. 2014. Auditory evoked potentials: Predicting speech therapy outcomes in children with phonological disorders. Clinics, 69, 212-8.

LOUSADA, M., JESUS, L. M. T., CAPELAS, S., MARGACA, C., SIMOES, D., VALENTE, A., HALL, A. and JOFFE, V. L. 2013. Phonological and articulation treatment approaches in Portuguese children with speech and language impairments: A randomized controlled intervention study. International Journal of Language \& Communication Disorders, 48, 172-87.

LOUSADA, M., JESUS, L. M. T., HALL, A. and JOFFE, V. 2014. Intelligibility as a clinical outcome measure following intervention with children with phonologically based speech-sound disorders. International Journal of Language \& Communication Disorders, 49, 584-601.

MACLEOD, A. A. N. and GLASPEY, A. M. 2014. A multidimensional view of gradient change in velar acquisition in three-year-olds receiving phonological treatment. Clinical Linguistics \& Phonetics, 28, 664-681. 
MAJOR, E. M. and BERNHARDT, B. 1998. Metaphonological skills of children with phonological disorders before and after phonological and metaphonological intervention. International Journal of Language \& Communication Disorders, 33, 413-444.

MASTERSON, J. J. and DANIELS, D. L. 1991. Motoric versus contrastive approaches to phonology therapy: A case study. Child Language Teaching and Therapy, 7, 127140.

MCINTOSH, B. and DODD, B. 2008. Evaluation of Core Vocabulary intervention for treatment of inconsistent phonological disorder: Three treatment case studies. Child Language Teaching and Therapy, 24, 307-327.

MCKEAN, K., PHILLIPS, B. and THOMPSON, A. 2012. A family-centred model of care in paediatric speech-language pathology. International Journal of SpeechLanguage Pathology, 14, 235-246.

MECROW, C., BECKWITH, J. and KLEE, T. 2010. An exploratory trial of the effectiveness of an enhanced consultative approach to delivering speech and language intervention in schools. International Journal of Language \& Communication Disorders, 45, 354-367.

MEZZOMO, C. L., MOTA, H. B., KESKE-SOARES, M., CERON, M. I. and DIAS, R. F. 2014. The influence of phonological awareness abilities in therapy for phonological disorder [A influência das habilidades em consciência fonológica na terapia para os desvios fonológicos]. Revista CEFAC, 16, 328-335.

MICCIO, A. W. and ELBERT, M. 1996. Enhancing stimulability: A treatment program. Journal of Communication Disorders, $29,335-351$.

MICCIO, A. W., ELBERT, M. and FORREST, K. 1999. The relationship between stimulability and phonological acquisition in children with normally developing and disordered phonologies. American Journal of Speech-Language Pathology, 8, 347-363.

MICCIO, A. W. and INGRISANO, D. R. 2000. The acquisition of fricatives and affricates: Evidence from a disordered phonological system. American Journal of SpeechLanguage Pathology, 9, 214-229.

MONAHAN, D. 1986. Remediation of common phonological processes: Four case studies. Language, Speech and Hearing Services in Schools, $17,199-206$.

MONTGOMERY, J. K. and BONDERMAN, R. 1989. Serving preschool children with severe phonological disorders. Language, Speech, and Hearing Services in Schools, $20,76-84$ 
MORRISETTE, M. L. and GIERUT, J. A. 2002. Lexical organization and phonological change in treatment. Journal of Speech, Language, and Hearing Research, 45, 14359.

MOTA, H. B., BAGETTI, T., KESKE-SOARES, M. and PEREIRA, L. F. 2005. A generalização baseada nas relações implicacionais em sujeitos submetidos à terapia fonológica [Generalization based on implicational relations in subjects treated with phonological therapy]. Pró-Fono Revista de Atualização Científica, 17, 99-110.

MOTA, H. B., KESKE-SOARES, M., BAGETTI, T., CERON, M. I. and FILHA, M. 2007. Análise comparativa da eficiência de três diferentes modelos de terapia fonológica [Comparative analyses of the effectiveness of three different phonological therapy models]. Pró-Fono Revista de Atualização Científica, 19, 67-74.

MURPHY, C. F. B., PAGAN-NEVES, L. O., WERTZNER, H. F. and SCHOCHAT, E. 2015. Children with speech sound disorder: comparing a non-linguistic auditory approach with a phonological intervention approach to improve phonological skills. Frontiers in Psychology, 6.

NELSON, H. D., NYGREN, P., WALKER, M. and PANOSCHA, R. 2006. Screening for speech and language delay in preschool children: Systematic evidence review for the US Preventive Services Task Force. Pediatrics, 117, e298-319.

OLSWANG, L. B. and BAIN, B. A. 1985. Monitoring phoneme acquisition for making treatment withdrawal decisions. Applied Psycholinguistics, 6, $17-37$.

PAGE, F., PERTILE, J.-A., TORRESI, K. and HUDSON, C. 1994. Alternative service delivery options: The effectiveness of intensive group treatment with pre-school children. Australian Journal of Human Communication Disorders, 22, 61-72.

PAGLIARIN, K. C., BRANCALIONI, A. R. and KESKE-SOARES, M. 2012. Terapia fonológica a partir da estimulação de sons isolados e em conjunto [phonological therapy based on the stimulation of single sounds and groups of sounds]. Revista da Sociedade Brasileira de Fonoaudiologia, 17, 23-7.

PAGLIARIN, K. C., MOTA, H. B. and KESKE-SOARES, M. 2009. Therapeutic efficacy analysis of three contrastive approach phonological models. Pró-Fono Revista de Atualização Científica, 21, 297-302.

PAGLIARIN, K. C., MOTA, H. B. and KESKE-SOARES, M. 2011. Generalização estrutural a partir do tratamento por diferentes modelos de oposições [Structural generalization after treatment based on different oppositions approaches]. Revista da Sociedade Brasileira de Fonoaudiologia, 16, $356-361$. 
PALLE, N., BERNTSSON, A., MINISCALCO, C. and PERSSON, C. 2014. The effectiveness of phonological intervention in preschool children: A single-subject design study. Logopedics, Phoniatrics, Vocology, 39, 19-29.

PAMPLONA, M. C., YSUNZA, A. and ESPINOSA, J. 1999. A comparative trial of two modalities of speech intervention for compensatory articulation in cleft palate children, phonologic approach versus articulatory approach. International Journal of Pediatric Otorhinolaryngology, 29, 21-26.

PAMPLONA, M. C., YSUNZA, A. and MORALES, S. 2014. Strategies for treating compensatory articulation in patients with cleft palate. International Journal of Biomedical Science, 10, 43-51.

PAMPLONA, M. C., YSUNZA, A. and RAMiREZ, P. 2004. Naturalistic intervention in cleft palate children. International Journal of Pediatric Otorhinolaryngology, 68, $75-81$.

PASCOE, M., STACKHOUSE, J. and WELLS, B. 2005. Phonological therapy within a psycholinguistic framework: Promoting change in a child with persisting speech difficulties. International Journal of Language \& Communication Disorders, 40, 189-220.

PENNEY, G., FEE, J. and DOWDLE, C. 1994. Vowel assessment and remediation: A case study. Child Language Teaching and Therapy, $10,47-66$.

PIERETTI, R. A., KAUL, S. D., ZARCHY, R. M. and O’HANLON, L. M. 2015. Using a multimodal approach to facilitate articulation, phonemic awareness, and literacy in young children. Communication Disorders Quarterly, 36, 131-141.

POLLOCK, K. E. 1983. Individual preferences: Case study of a phonologically delayed child. Topics in Language Disorders, 3, 10-23.

POWELL, T. W. 1991. Planning for phonological generalisation: An approach to treatment target selection. American Journal of Speech-Language Pathology, 1, 21-27.

POWELL, T. W. 1993. Phonetic inventory constraints in young children: Factors affecting acquisition patterns during treatment. Clinical Linguistics \& Phonetics, 7, 45-57.

POWELL, T. W. and ELBERT, M. 1984. Generalisation following the remediation of early- and later-developing consonant clusters. Journal of Speech and Hearing Disorders, 49, 211-218.

POWELL, T. W., ELBERT, M. and DINNSEN, D. A. 1991. Stimulability as a factor in the phonological generalisation of misarticulating preschool children. Journal of Speech and Hearing Research, 34, 1318-1328. 
POWELL, T. W., ELBERT, M., MICCIO, A. W., STRIKE-ROUSSOS, C. and BRASSEUR, J. 1998. Facilitating [s] production in young children: An experimental evaluation of motoric and conceptual treatment approaches. Clinical Linguistics \& Phonetics, 12, 127-146.

RAY, J. 2002. Treating phonological disorders in a multilingual child: A case study. American Journal of Speech-Language Pathology, 11, 305-315.

ROBB, M. P., BLEILE, K. M. and YEE, S. S. L. 1999. A phonetic analysis of vowel errors during the course of treatment. Clinical Linguistics \& Phonetics, 13 , 309-321.

RUDOLPH, J. M. and WENDT, O. 2014. The efficacy of the cycles approach: A multiple baseline design. Journal of Communication Disorders, 47, 1-16.

RUSCELLO, D. M., CARTWRIGHT, L. R., HAINES, K. B. and SHUSTER, L. I. 1993. The use of different service delivery models for children with phonological disorders. Journal of Communication Disorders, 26, 193-203.

RVACHEW, S. 1994. Speech perception training can facilitate sound production learning. Journal of Speech and Hearing Research, 37, 347-357.

RVACHEW, S. and BERNHARDT, B. M. 2010. Clinical implications of dynamic systems theory for phonological development. American Journal of Speech-Language Pathology, 19, 34-50.

RVACHEW, S. and BROSSEAU-LAPRÉ, F. 2015. A randomized trial of 12-week interventions for the treatment of developmental phonological disorder in francophone children. American Journal of Speech-Language Pathology, 24, 637-658.

RVACHEW, S. and NOWAK, M. 2001. The effect of target-selection strategy on phonological learning. Journal of Speech, Language, and Hearing Research, 44, 610-623.

RVACHEW, S., NOWAK, M. and CLOUTIER, G. 2004. Effect of phonemic perception training on the speech production and phonological awareness skills of children with expressive phonological delay. American Journal of Speech-Language Pathology, 13, 250-263.

RVACHEW, S., RAFAAT, S. and MARTIN, M. 1999. Stimulability, speech perception skills, and the treatment of phonological disorders. American Journal of SpeechLanguage Pathology, 8, 33-43.

SABEN, C. B. and COSTELLO INGHAM, J. 1991. The effects of minimal pairs treatment on the speech-sound production of two children with phonologic disorders. Journal of Speech and Hearing Research, 14, 1023-1040. 
SEEFF-GABRIEL, B., CHIAT, S. and PRING, T. 2012. Intervention for co-occurring speech and language difficulties: Addressing the relationship between the two domains. Child Language Teaching and Therapy, 28, 123-135.

SHEA, R. L. and TYLER, A. A. 2001. The effectiveness of a prosodic intervention on children's metrical patterns. Child Language Teaching and Therapy, 17, 55-76.

SHILLER, D. M., RVACHEW, S. and BROSSEAU-LAPRÉ, F. 2010. Importance of the auditory perceptual target to the achievement of speech production accuracy. Canadian Journal of Speech-Language Pathology and Audiology, 34.

SHOAF, K. O., IYER, S. N. and BOTHE, A. K. 2009. Using a single-subject experimental design to implement a nonlinear phonology approach to target selection. Contemporary Issues in Communication Sciences and Disorders, 36, 77-88.

SHRIBERG, L. D. and KWIATKOWSKI, J. 1982. Phonological disorders II: A conceptual framework for management. Journal of Speech and Hearing Disorders, 47, 24256.

SHRIBERG, L. D. and KWIATKOWSKI, J. 1987. A retrospective study of spontaneous generalisation in speech-delayed children. Language, Speech, and Hearing Services in Schools, 18, 144-157.

SHRIBERG, L. D. and KWIATKOWSKI, J. 1990. Self-monitoring and generalization in preschool speech-delayed children. Language, Speech and Hearing Services in Schools, 21, 157-170.

SHRIBERG, L. D., KWIATKOWSKI, J. and SNYDER, T. 1989. Tabletop versus microcomputer-assisted speech management: Stabilization phase. Journal of Speech and Hearing Disorders, 54, 233-248.

SHRIBERG, L. D., KWIATKOWSKI, J. and SNYDER, T. 1990. Tabletop versus microcompter assisted speech management: Response evocation phase. Journal of Speech and Hearing Disorders, 55, 635-655.

SMITH, J., DOWNS, M. and MOGFORD-BEVAN, K. 1998. Can phonological awareness training facilitation minimal pair therapy? International Journal of Language \& Communication Disorders, 33, 463. 
SOMMERS, R. K., LOGSDON, B. and WRIGHT, J. 1992. A review and critical analysis of treatment research related to articulation and phonological disorders. Journal of Communication Disorders, 25, 3-22.

SPEAKE, J., STACKHOUSE, J. and PASCOE, M. 2012. Vowel targeted intervention for children with persisting speech difficulties: Impact on intelligibility. Child Language Teaching and Therapy, 28, 277-295.

STOEL-GAMMON, C., STONE-GOLDMAN, J. and GLASPEY, A. 2002. Pattern-based approaches to phonological therapy. Seminars in Speech and Language, 23, 3-14.

STRINGFELLOW, K. and MCLEOD, S. 1994. Using a facilitating phonetic context to reduce an unusual form of gliding. Language, Speech, and Hearing Services in Schools, 25, 191-193.

SUGDEN, E., BAKER, E., MUNRO, N. and WILLIAMS, A. L. 2016. Involvement of parents in intervention for childhood speech sound disorders: A review of the evidence. International Journal of Language \& Communication Disorders, 51, 597-625.

TOPBAŞ, S. and ÜNAL, Ö. 2010. An alternating treatment comparison of minimal and maximal opposition sound selection in Turkish phonological disorders. Clinical Linguistics \& Phonetics, 24, 646-668.

TYLER, A. A. 1995. Durational analysis of stridency errors in children with phonological impairment. Clinical Linguistics \& Phonetics, 9, 211-228.

TYLER, A. A., EDWARDS, M. L. and SAXMAN, J. H. 1987. Clinical application of two phonologically based treatment procedures. Journal of Speech and Hearing Disorders, 52, 393-409.

TYLER, A. A., EDWARDS, M. L. and SAXMAN, J. H. 1990. Acoustic validation of phonological knowledge and its relationship to treatment. Journal of Speech and Hearing Disorders, 55, 251-261.

TYLER, A. A. and FIGURSKI, G. R. 1994. Phonetic inventory changes after treating distinctions along an implicational hierarchy. Clinical Linguistics \& Phonetics, 8, $91-$ 107.

TYLER, A. A., FIGURSKI, G. R. and LANGSDALE, T. 1993. Relationships between acoustically determined knowledge of stop place and voicing contrasts and phonological treatment progress. Journal of Speech and Hearing Research, 36, 746-759. 
TYLER, A. A., GILLON, G., MACRAE, T. and JOHNSON, R. L. 2011. Direct and indirect effects of stimulating phoneme awareness vs. other linguistic skills in preschoolers with co-occurring speech and language impairments. Topics in Language Disorders, 31, 128-144.

TYLER, A. A. and LEWIS, K. E. 2005. Relationships among consistncy/variability and other phonological measures over time. Topics in Language Disorders, 25 , $243-253$.

TYLER, A. A., LEWIS, K. E., HASKILL, A. and TOLBERT, L. C. 2002. Efficacy and cross-domain effects of a morphosyntax and a phonology intervention. Language, Speech, and Hearing Services in Schools, 33, 52-66.

TYLER, A. A., LEWIS, K. E., HASKILL, A. and TOLBERT, L. C. 2003a. Outcomes of different speech and language goal attack strategies. Journal of Speech, Language, and Hearing Research, 46, 1077-1094.

TYLER, A. A., LEWIS, K. E. and WELCH, C. M. 2003b. Predictors of phonological change following intervention. American Journal of Speech-Language Pathology, 12, $289-98$.

TYLER, A. A. and SANDOVAL, K. T. 1994. Preschoolers with phonological and language disorders: Treating different linguistic domains. Language, Speech, and Hearing Services in Schools, 25, 215-234.

TYLER, A. A. and WATTERSON, K. H. 1991. Effects of phonological versus language intervention in preschoolers with both phonological and language impairment. Child Language Teaching and Therapy, 7, 141-160.

TYLER, A. A., WILLIAMS, M. J. and LEWIS, K. E. 2006. Error consistency and the evaluation of treatment outcomes. Clinical Linguistics \& Phonetics, 20, 411-422.

VAN BYSTERVELDT, A. K., GILLON, G. and FOSTER-COHEN, S. 2010. Integrated speech and phonological awareness intervention for pre-school children with Down syndrome. International Journal of Language \& Communication Disorders, 45, 320-335.

VAN BYSTERVELDT, A. K., GILLON, G. and FOSTER-COHEN, S. 2014. A phonological awareness intervention case study of a child with Down syndrome. Speech, Language and Hearing, 17, 25-36.

WATERS, D., HAWKES, C. and BURNETT, E. 1998. Targeting speech processing strengths to facilitate pronunciation change. International Journal of Language \& Communication Disorders, 33, 469-474. 
WEINER, F. 1981. Treatment of phonological disability using the method of meaningful minimal contrast: Two case studies. Journal of Speech and Hearing Disorders, 46, 97-103.

WILLIAMS, A. L. 1991. Generalisation patterns associated with training least phonological knowledge. Journal of Speech and Hearing Research, 34, 722-733.

WILLIAMS, A. L. 1993. Phonological reorganization: A qualitative measure of phonological improvement. American Journal of Speech-Language Pathology, 2, 44-51.

WILLIAMS, A. L. 2000. Multiple oppositions: Case studies of variables in phonological intervention. American Journal of Speech-Language Pathology, 9, $289-299$.

WILLIAMS, A. L. 2005. Assessment, target selection and intervention: Dynamic interactions within a systemic perspective. Topics in Language Disorders, $25,231-242$.

WILLIAMS, A. L. 2012. Intensity in phonological intervention: Is there a prescribed amount? International Journal of Speech-Language Pathology, 14, 456-461.

WOLFE, V., BLOCKER, S. D. and PRATER, N. J. 1988. Articulatory generalisation in two word-medial, ambisyllabic contexts. Language, Speech, and Hearing Services in Schools, 19, 251-258.

WOLFE, V., PRESLEY, C. and MESARIS, J. 2003. The importance of sound identification training in phonological intervention. American Journal of Speech-Language Pathology, 12, 282-288.

WREN, Y. and ROULSTONE, S. 2008. A comparison between computer and tabletop delivery of phonology therapy. International Journal of Speech-Language Pathology, $10,346-363$.

YODER, P., CAMARATA, S. and GARDNER, E. 2005. Treatment effects on speech intelligibility and length of utterance in children with specific language and intelligibility impairments. Journal of Early Intervention, 28, 34-49.

YODER, P., CAMARATA, S. and WOYNAROSKI, T. 2016. Treating speech comprehensibility in students with down syndrome. Journal of Speech, Language, and Hearing Research, 59, 446-459.

YOUNG, E. C. 1987. The effects of treatment on consonant cluster and weak syllable reduction processes in misarticulating children. Language, Speech, and Hearing Services in Schools, 18, 23-33. 


\section{Appendix B: Questions Regarding Service Delivery, Intervention Approaches, and Intervention Intensity}

\section{Questions Regarding Service Delivery and Intervention Approaches}

1. Which of the following intervention models do you use when working with children with phonology-based speech sound disorders? Select ALL that apply

- Direct models (i.e., speech pathologist working directly with the child)

- Indirect models (i.e., speech pathologist working in consultation with others such as parents and/or teachers to support the child)

- Community education (e.g., raising awareness of phonology-based speech sound disorders)

- "Watch and wait" (e.g., monitoring a child's speech development to determine if intervention is necessary)

- Other (please specify)

2. Please indicate which is your MOST COMMON intervention model for children with phonology-based speech sound disorders:

- Direct models

- Indirect models

- Community education

- "Watch and wait"

- Other

3. Which of the following methods of service delivery do you use in intervention for children with phonology-based speech sound disorders? Select ALL that apply

- Individual intervention

- Group intervention

- Parent training

- Home program (e.g., a selection of worksheets/activities provided to the family for completion at home with minimal continued input from the speech pathologist)

- Telehealth

- Teacher training

- Classroom-based therapy

- Computer-based therapy (e.g. iPad, computer programs — excludes telehealth)

- Community education

- Other (please specify)

4. Please select the MOST COMMON method of service delivery you use to provide intervention to children with phonology-based speech sound disorders:

- Individual intervention

- Group intervention

- Parent training

- Home program (e.g., a selection of worksheets/activities provided to the family for completion at home with minimal continued input from the speech pathologist)

- Telehealth

- Teacher training

- Classroom-based therapy

- Computer-based therapy (e.g. iPad, computer programs - excludes telehealth)

- Community education

- Other 
5. Where do you provide intervention to children with phonology-based speech sound disorders? Select ALL that apply

- Community health/hospital clinic setting

- Private practice clinic setting

- Early childhood/preschool setting

- School setting

- Client's home

- Other (please specify)

6. Please select the MOST COMMON place where you provide intervention to children with phonology-based speech sound disorders:

- Community health/hospital clinic setting

- Private practice clinic setting

- Early childhood/preschool setting

- School setting

- Client's home

- Other

7. Who directly provides the intervention to the children on your caseload with phonology-based speech sound disorders? Select ALL that apply

- Speech pathologist

- Preschool or classroom teacher

- Parents or caregivers

- Teacher's aide

- Speech pathology assistant

- Learning support teacher/itinerant support teacher

- Supervised speech pathology student

- Interpreter

- Other allied health professional

- Other (please specify)

8. Please indicate the MOST COMMON intervention provider:

- Speech pathologist

- Preschool or classroom teacher

- Parents or caregivers

- Teacher's aide

- Speech pathology assistant

- Learning support teacher/itinerant support teacher

- Supervised speech pathology student

- Interpreter

- Other allied health professional

- Other

The following questions relate to the intervention you provide to children with phonologybased speech sound disorders

9. When selecting targets for intervention, which types of targets do you usually select?

- Developmental targets (e.g. stimulable sounds, earlier developing sounds, early phonological processes)

- Non-developmental targets (e.g. non-stimulable sounds, later developing sounds, later phonological processes)

- Collapse of contrast targets (e.g. multiple sounds that a child produces as the same sound)

- Other (please specify) 
10. How do you USUALLY treat phonology-based speech sound disorders?

- You focus on the production of speech sounds in isolation, syllables, words, phrases, sentences then conversation

- You focus on error patterns (starting at word level) and the communicative functions of speech sounds in words

- Other (please provide details)

11. Please indicate which of the following tasks you USUALLY include in intervention for phonology-based speech sound disorders. Select ALL that apply

- Production tasks (e.g. the child produces their target sounds)

- Perceptual tasks (e.g. auditory awareness activities, such as auditory bombardment or auditory discrimination)

- Conceptual tasks (e.g. activities designed to develop the child's awareness of the features of target sounds, such as long/short sounds or rhyming features)

- Other (please specify)

12. Please select which of the following treatment methods you USUALLY use for children with phonology-based speech sound disorders. Select ALL that apply

- Auditory bombardment/stimulation (e.g. Hodson \& Paden, 1991)

- Auditory discrimination (e.g. Berry \& Eisenson, 1956)

- Core vocabulary (e.g. Dodd \& Bradford, 2000)

- Cued articulation (e.g. Passey, 1990)

- Cycles (e.g. Hodson \& Paden, 1983)

- Imagery approach (e.g. Klein, 1996)

- Maximal oppositions contrast (e.g. Gierut, 1990)

- Metaphon (e.g. Howell \& Dean, 1984)

- Minimal oppositions contrast (Minimal Pairs) (e.g. Weiner, 1981)

- Multiple oppositions contrast (e.g. Williams, 2000)

- Non-speech oromotor intervention (e.g. Lancaster \& Pope, 1989)

- Nuffield Centre Dyspraxia Programme (e.g. Nuffield Hearing and Speech Centre, 2004)

- Parents and Children Together (PACT) (e.g. Bowen \& Cupples, 1999)

- Phonological awareness (e.g. Gillon, 2000)

- Prompts for Restructuring Oral Muscular Phonetic Targets (e.g. PROMPT) (e.g. Hayden, 2006)

- Traditional articulation therapy (e.g. van Riper, 1939)

- Whole language therapy (e.g. Hoffman, Norris \& Monjure, 1990)

- Other (please specify)

\section{Questions on Intervention Intensity}

This section refers to the intensity of the direct intervention you provide to a child with a phonology-based speech sound disorder.

We define intensity in keeping with Warren, Fey \& Yoder (2007), including:

- Session duration (how long each session lasts)

- Dose (the number of teaching moments in each session [e.g. the number of opportunities for production practice, auditory bombardment and/or phonological awareness tasks in a session])

- Session frequency (how often sessions occur), and;

- Total intervention duration (length of stay the overall time from the first therapy session to discharge) 
Please answer the following questions in relation to your main service delivery context.

1. Session duration: how long are your MOST COMMON intervention sessions for children with phonology based speech sound disorders?

- Less than 30 minutes

- $\quad 30$ to 44 minutes

- 45 to 59 minutes

- 60 to 89 minutes

- 90 minutes or longer

2. Dose: within your MOST COMMON intervention sessions, how many opportunities does a child with a phonology-based speech sound disorder receive to PRODUCE their speech targets?

- None

- Less than 20

- 21-49

- $\quad 50-99$

- $100-149$

- $150-199$

- $200+$

- Unsure

3. Dose: within your MOST COMMON intervention sessions, how many opportunities does a child with a phonology-based speech sound disorder receive to complete PERCEPTUAL tasks (such as auditory awareness and auditory discrimination tasks)?

- None

- Less than 20

- 21-49

- $50-99$

- 100-149

- $150-199$

- $200+$

- Unsure

4. Dose: within your MOST COMMON intervention sessions, how many opportunities does a child with a phonology-based speech sound disorder receive to complete CONCEPTUAL tasks (such as phonological awareness and metaphonological tasks)?

- None

- $\quad$ Less than 20

- 21-49

- $50-99$

- 100-149

- 150-199

- $200+$

- Unsure

5. Session frequency: how frequently do you USUALLY schedule sessions for children with phonology-based speech sound disorders?

- Less than 1 session per month

- One session per month

- 1 to 2 sessions per month

- 1 x weekly

- 2 x weekly

- 3 x weekly 
- More than 3 x weekly

6. Total intervention duration: how long (in weeks or months), is the typical length of stay for children with phonology-based speech sound disorders (i.e. how many weeks or months from the initial intervention session to discharge)? Please specify if it is in weeks or months.

7. Total intervention duration: how many sessions do children with phonology-based speech sound disorders typically receive over their length of stay (i.e. how many sessions does the child receive from the initial intervention session to discharge)?

8. Do the majority of children on your caseload with phonology-based speech sound disorders receive the same intervention intensity?

- Yes

- No

9. Do you provide block on/block off therapy to children with phonology-based speech sound disorders (e.g. the child receives 10 weeks of therapy then receives a 10 week break, then recommences 10 weeks of therapy)?

- Yes

- No

- Sometimes

The following questions (Questions $10-13$ ) were only asked of participants who reported that they provide (or sometimes provide) block on/block off therapy:

10. Approximately what percentage of children with phonology-based speech sound disorders on your caseload receive block therapy?

- None

- Less than $10 \%$

- Between $10 \%$ and $39 \%$

- Between 40 and 70\%

- Greater than $70 \%$

- Unsure

11. What is the duration of the "on" block (in weeks)?

12. What is the duration of the "off" block (in weeks)?

13. Please describe the frequency and duration of the intervention sessions in the "on' blocks.

The intervention intensity provided to children with phonology-based speech sound disorders can be influenced by several factors, including workplace, clinician and client factors.

14. Which of the following WORKPLACE factors influence the intervention intensity that you provide to children with phonology-based speech sound disorders (i.e. the frequency of sessions, the duration of sessions, and the total intervention duration)? Select ALL that apply

- Waiting lists

- Scheduling of intervention around other workplace commitments

- Workplace policy

- Size of current active caseload

- Funding reasons

- $\quad$ Service delivery model

- Other (please specify)

15. Which of the following CLINICIAN factors influence the intervention intensity that you provide to children with phonology-based speech sound disorders (i.e. the 
frequency of sessions, the duration of sessions, and the total intervention duration)?

Select ALL that apply

- Personal factors (e.g. you only work one day per week)

- Your application of research evidence around recommended intervention intensities

- You are implementing an intervention program that specifies intervention intensity

- Previous experience with similar clients

- You have always provided this level of intervention intensity

- Other (please specify)

16. Which of the following CLIENT factors influence the intervention intensity that you provide to children with phonology-based speech sound disorders (e.g. the frequency of sessions, the duration of sessions, and the total intervention duration)? Select ALL that apply

- Funding reasons (e.g. Medicare rebates, or affordability of ongoing private practice)

- Rate of progress in therapy

- Family preferences

- Severity of phonology-based speech sound disorder

- Travel time (e.g. client lives close to/far away from the service)

- Age of client

- Cultural and/or linguistic background of client/client's family

- Other (please specify)

17. Between workplace, clinician and client factors, which has the biggest influence on the intervention intensity that you provide to children with phonology-based speech sound disorders?

- Workplace factors

- Clinician factors

- Client factors

18. For a preschool child with a moderate-severe phonology-based speech sound disorder, what would be your ideal direct intervention intensity?

- Frequency of sessions

- Duration of each session

- Total number of sessions

- Total intervention duration (the time period, in weeks or months or years, over which the child receives intervention) 